\title{
Chapter 4 \\ Impedance and Collective Effects
}

\author{
E. Metral, G. Rumolo, and W. Herr
}

As the beam intensity increases, the beam can no longer be considered as a collection of non-interacting single particles: in addition to the "single-particle phenomena", "collective effects" become significant. At low intensity a beam of charged particles moves around an accelerator under the Lorentz force produced by the "external" electromagnetic fields (from the guiding and focusing magnets, RF cavities, etc.). However, the charged particles also interact with themselves (leading to space charge effects) and with their environment, inducing charges and currents in the surrounding structures, which create electromagnetic fields called wake fields. In the ultra-relativistic limit, causality dictates that there can be no electromagnetic field in front of the beam, which explains the term "wake". It is often useful to examine the frequency content of the wake field (a time domain quantity) by performing a Fourier transformation on it. This leads to the concept of impedance (a frequency domain quantity), which is a complex function of frequency. The charged particles can also interact with other charged particles present in the accelerator (leading to two-stream effects, and in particular to electron cloud effects in positron/hadron machines) and with the counter-rotating beam in a collider (leading to beam-beam effects). As the beam intensity increases, all these "perturbations" should be properly quantified and the motion of the charged particles will eventually still be governed by the Lorentz force but using the total electromagnetic fields, which are the sum of the external and perturbation fields. Note that in some cases a perturbative treatment is not sufficient and the problem has to be solved self consistently. These perturbations can lead to both incoherent (i.e. of

Coordinated by E. Metral

E. Metral $(\bowtie) \cdot$ G. Rumolo $\cdot$ W. Herr

CERN (European Organization for Nuclear Research), Geneva, Switzerland

e-mail: Elias.Metral@cern.ch; Giovanni.Rumolo@cern.ch; Werner.Herr@cern.ch

(C) The Author(s) 2020

S. Myers, H. Schopper (eds.), Particle Physics Reference Library,

https://doi.org/10.1007/978-3-030-34245-6_4 
a single particle) and coherent (i.e. of the centre of mass) effects, in the longitudinal and in one or both transverse directions, leading to beam quality degradation or even partial or total beam losses. Fortunately, stabilising mechanisms exist, such as Landau damping, electronic feedback systems and linear coupling between the transverse planes (as in the case of a transverse coherent instability, one plane is usually more critical than the other).

Beam instabilities cover a wide range of effects in particle accelerators and they have been the subjects of intense research for several decades. As the machines performance was pushed new mechanisms were revealed and nowadays the challenge consists in studying the interplays between all these intricate phenomena, as it is very often not possible to treat the different effects separately $[1,2]$. This field is still very active as can be revealed by the recent (and future) international workshops devoted to this subject [3-5].

This chapter is structured as follows: space charge is discussed in Sect. 4.1, wake fields (and related impedances) in Sect. 4.2, the induced coherent instabilities in Sect. 4.3 and the Landau damping mechanism in Sect. 4.4. The two-stream effects are analyzed in Sect. 4.5, concentrating mainly on electron cloud, while beam-beam effects are reviewed in Sect. 4.6, before concluding in Sect. 4.7 by the numerical modelling of collective effects.

\title{
4.1 Space Charge
}

\author{
E. Metral
}

\subsubsection{Direct Space Charge}

Two space charge effects are distinguished: the direct space charge and the indirect (or image) one ([6-9], and references therein). The direct space charge comes from the interaction between the particles of a single beam, without interaction with the surrounding vacuum chamber. Consider two particles with the same charge (for instance protons) in vacuum. They will feel two forces: the Coulomb repulsion (as they have the same charge) and the magnetic attraction (as they represent currents moving in the same direction, leading to an azimuthal magnetic field). Let's assume that particle 1 is moving with speed $v_{1}=\beta_{1} c$ with respect to the laboratory frame, with $\beta$ the relativistic velocity factor and $c$ the speed of light. In its rest frame, particle 1 produces only an electrostatic field, which can be computed, and applying the relativistic transformation of the electromagnetic fields between the rest and laboratory frames, the magnetic contribution can be obtained. Note that there is no magnetic contribution in the longitudinal plane $\left(B_{s}=0\right)$, which leads to the longitudinal Lorentz force $F_{s}=e E_{s}$, where $e$ is the elementary charge, $s$ the azimuthal coordinate and $E_{s}$ the longitudinal electric field. The transverse 
(horizontal and vertical) Lorentz force on particle 2, moving with speed $v_{2}=\beta_{2} c$ with respect to the laboratory frame, is written

$$
F_{x, y}=e E_{x, y}\left\{\begin{array}{ll}
\left(1-\beta_{1} \beta_{2}\right), & \text { if } 2 \text { moves in same direction as } 1 \\
\left(1+\beta_{1} \beta_{2}\right), & \text { if } 2 \text { moves in opposite direction as } 1
\end{array} .\right.
$$

The first case corresponds to the space charge case where both particles move in the same direction, while the second corresponds to the beam-beam case (see Sect. 4.6) where the particles move in opposite direction. In both cases, the first term comes from the electric field while the second comes from the magnetic one. The main difference between the two regimes is that for the space charge case there is a partial compensation of the two forces, while for the beam-beam case the two forces add. The space charge force is maximum at low energy and vanishes at high energy, while the beam-beam force is maximum at high energy. Considering the space charge regime and assuming the same speed for both beams, the Lorentz force simplifies to

$$
F_{x, y}=e E_{x, y}\left(1-\beta^{2}\right)=e \frac{E_{x, y}}{\gamma^{2}}, \quad F_{s}=e E_{s},
$$

where $\gamma$ is the relativistic mass factor. Assuming a circular beam pipe with radius $b$ (which is important only for the computation of the longitudinal force) and applying Gauss's law, the electromagnetic fields can be computed for a bunch with Gaussian radial density (with rms $\sigma_{x}=\sigma_{y}=\sigma$ ) using the cylindrical coordinates $(r, \theta, s)$. The associated Lorentz forces are given by

$$
F_{r}=\frac{e}{\gamma^{2}} E_{r}=\frac{e \lambda(z)}{2 \pi \varepsilon_{0} \gamma^{2}}\left(\frac{1-\mathrm{e}^{-\frac{r^{2}}{2 \sigma^{2}}}}{r}\right), \quad F_{s}=-\frac{e}{2 \pi \varepsilon_{0} \gamma^{2}} \frac{d \lambda(z)}{d z} \int_{r^{\prime}=r}^{b} \frac{1-\mathrm{e}^{-\frac{r^{2}}{2 \sigma^{2}}}}{r^{\prime}} d r^{\prime}
$$

where $\lambda(z)$ is the longitudinal line density, $z=s-v t$ with $t$ being the time, and $\varepsilon_{0}$ the vacuum permittivity. A first observation is that the space charge forces are highly nonlinear. Another important observation is that the radial force is proportional to the longitudinal density while the longitudinal one is proportional to the derivative of the longitudinal density. Linearizing both forces (for very small amplitudes where $r<\sigma$ ) leads to

$$
F_{r} \approx \frac{e \lambda(z)}{2 \pi \varepsilon_{0} \gamma^{2}} \frac{r}{2 \sigma^{2}}, \quad F_{s} \approx-\frac{e}{4 \pi \varepsilon_{0} \gamma^{2}} \frac{d \lambda(z)}{d z}\left[1+2 \ln \left(\frac{b}{\sqrt{2} \sigma}\right)\right] .
$$

This means that the transverse space charge force is linear for small amplitudes and defocusing. Due to the additional space charge force, e.g. the horizontal betatron tune will no longer be the unperturbed tune $Q_{x 0}$ but will be $Q_{x}=Q_{x 0}+\Delta Q_{x}$, where $\Delta Q_{x}$ is the horizontal incoherent betatron tune shift. Similarly, the new synchrotron 
tune will be $Q_{s}=Q_{s 0}+\Delta Q_{s}$, where $\Delta Q_{s}$ is the incoherent synchrotron tune shift. The betatron and synchrotron linearized incoherent space charge tune shifts are given by

$$
\Delta Q_{x}^{\mathrm{Lin}}=-\frac{N_{b} r_{p}}{4 \pi \beta \gamma^{2} \varepsilon_{x, \mathrm{rms}}^{\text {norm } B}}, \quad \Delta Q_{s}^{\mathrm{Lin}}=+\frac{\eta N_{b} e^{2} R^{2}}{8 \pi \sqrt{2 \pi} \varepsilon_{0} E_{\mathrm{total}} \beta^{2} \gamma^{2} \sigma_{z}^{3} Q_{s 0}}\left[1+2 \ln \left(\frac{b}{\sqrt{2} \sigma}\right)\right],
$$

where $N_{b}$ is the number of protons in the bunch, $r_{p}$ the classical proton radius, $\varepsilon_{x, \mathrm{rms}}^{\mathrm{norm}}=\beta \gamma \varepsilon_{x, \mathrm{rms}}$ the normalized rms horizontal emittance, with $\varepsilon_{x, \mathrm{rms}}=\sigma^{2} / \beta_{x}$ at a place of zero dispersion with $\beta_{x}$ the horizontal betatron function, $B=\sqrt{2 \pi} \sigma_{z} / 2 \pi R$ is the bunching factor (assuming a Gaussian longitudinal distribution with $\mathrm{rms} \sigma_{z}$ ) with $R$ the average machine radius, $\eta=\gamma_{\mathrm{tr}}^{-2}-\gamma^{-2}$ the slip factor (where $\gamma_{\mathrm{tr}}$ stands for $\gamma$ at transition energy) and $E_{\text {total }}$ is the total particles' energy. It is shown from Eq. (4.5) that the transverse betatron tune shift is always negative, revealing that the space charge force is always defocusing. Note that for the case of an ion with mass number $A$ and charge state $Z$, the transverse tune shift has to be multiplied by $Z^{2} / A$.

Contrary to the transverse case, the longitudinal space charge is defocusing below transition (as $\eta<0$ ) and focusing above (as $\eta>0$ ). One can therefore already anticipate some longitudinal mismatch issues when crossing transition with highintensity bunches, i.e. the bunch length will not be in equilibrium anymore and will oscillate inside the RF buckets. Such a case is depicted in Fig. 4.1(left) for the particular case of the high-intensity bunch in the CERN PS machine, which is sent to the nTOF (neutron Time-Of-Flight) experiment [10]. The computed quadrupolar oscillation is induced when transition is crossed and is a consequence of the longitudinal mismatch: below transition space charge is defocusing which reduces the bucket height and increases the bunch length, while above transition space charge is focusing which increases the bucket height and decreases the bunch length.
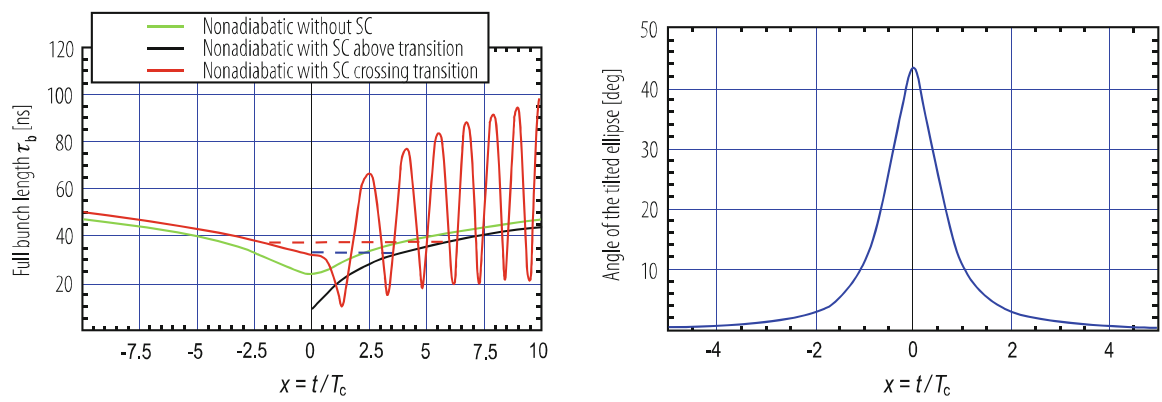

Fig. 4.1 (Left) Computation of the evolution of the full bunch length vs. time for the case of the nonadiabatic theory (the adiabatic theory is not valid anymore close to transition) with and without space charge, applied to the PS nTOF bunch [10]. $T_{\mathrm{c}}$ is the nonadiabatic time, equal to $\sim 2 \mathrm{~ms}$ in the present case. (Right) Evolution of the angle of the tilted ellipse around transition (without space charge) 
Therefore, there is an intensity-dependent step in the equilibrium bunch length at transition, which leads to a longitudinal mismatch and subsequent quadrupolar oscillations. If these bunch shape oscillations are not damped they will eventually result in filamentation and longitudinal emittance blow-up. It's worth mentioning that in presence of significant space charge, the minimum of bunch length is not reached right at transition anymore, but after about one nonadiabatic time $T_{\mathrm{c}}$, i.e. after $\sim 2 \mathrm{~ms}$ in the present case [11, 12]. The same kind of mechanism appears with the inductive part of the longitudinal machine impedance (see Sect. 4.2). The only difference is that in this case, the equilibrium bunch length is shorter below transition and longer above transition.

If transition crossing cannot be avoided, the $\gamma_{\mathrm{t}}$ jump is the only (known) method to overcome all the intensity limitations. It consists in an artificial increase of the transition crossing speed by means of fast pulsed quadrupoles. The idea is that quadrupoles at nonzero dispersion locations can be used to adjust the momentum compaction factor. The change in momentum compaction (called $\gamma_{\mathrm{t}}$ jump) depends on the unperturbed and perturbed dispersion functions at the kick-quadrupole locations. These schemes were pioneered by the CERN PS group [13-16]. Such a $\gamma_{t}$ jump scheme makes it possible to keep the beam at a safe distance from transition, except for the very short time during which the transition region is crossed at a speed increased by one or two orders of magnitude. Looking at Fig. 4.1(left) clearly reveals why an asymmetric jump was proposed in the past [14] to damp the longitudinal quadrupolar oscillations arising from the space charge induced mismatch: the idea is to jump rapidly from an equilibrium bunch length below transition to the same value above. The amplitude of the jump is defined by the time needed to go to the same equilibrium bunch length above transition. The minimum amplitude of the jump corresponds to the case represented with the dashed blue line starting right at transition. However, in this case the initial longitudinal phase space ellipse is tilted (see Fig. 4.1(right)), while the final one is almost not, which is not ideal. One might want therefore to start the jump earlier, when the longitudinal phase space is almost not tilted, for instance at $x \approx-2$, which requires a larger jump (see the dashed orange line in Fig. 4.1(left)).

Coming back to the transverse space charge, in the case of an elliptical beam (instead of a round one), one has to replace $2 \sigma_{x}^{2}$ by $\sigma_{x}\left(\sigma_{x}+\sigma_{y}\right)$ and $2 \sigma_{y}{ }^{2}$ by $\sigma_{y}\left(\sigma_{x}+\sigma_{y}\right)$. Furthermore, due to the nonlinear nature of the space charge, the tune shifts of the different particles will not be the same, which will lead to a tune spread: plotted in the tune diagram it is called a tune footprint. The latter has to be accommodated in the tune diagram, without crossing harmful resonance lines, which might lead to emittance growth and/or beam losses. The exact tune footprint depends on the distribution and to illustrate this effect we consider in the following a round beam with quasi-parabolic distribution function, whose particle density extends up to $\sim 3.2 \sigma[17,18]$. The corresponding horizontal 2D (i.e. neglecting the longitudinal distribution) space charge force is plotted in Fig. 4.2(left), and the tune footprint in Fig. 4.2(right). The unperturbed (low-intensity) working point is in the top right corner, the small-amplitude particles have the largest tune shifts while the largest amplitude particles have the smallest tune shifts. If the longitudinal 

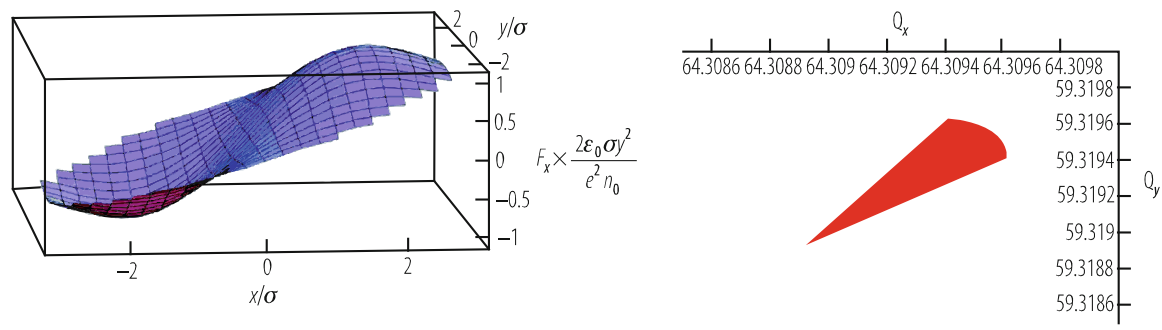

Fig. 4.2 Horizontal 2D (i.e. neglecting the longitudinal distribution) space charge force (left) and tune footprint for the case of the CERN LHC at injection, assuming the tunes in collision (64.31, 59.32). The parameter $n_{0}$ is the constant term in the particle density [18]

distribution is taken into account, the longitudinal variation (due to synchrotron oscillations) of the transverse space-charge force fills the gap until the low-intensity working point. However, it is interesting to plot it like this to clearly see the region occupied by the large synchrotron amplitude particles, because the interaction with a nonlinear resonance will depend on the overlapping position. Several possibilities exist with core emittance blow-up, creation of tails and/or beam losses. In particular, if the resonance interacts with the small amplitude particles, there could be a regime of loss-free (core-)emittance blow-up, while if the resonance interacts with the particles with large synchrotron amplitudes (i.e. if the resonance line is in the gap between the 2D tune footprint and the low-intensity working point) there could be a regime with continuous loss due to the trapping-detrapping mechanisms, as observed both in the PS [19] and at SIS18 [20].

Finally, another space charge mechanism, which could be important in highintensity synchrotrons with unsplit transverse tunes (i.e. having the same integer) is the Montague resonance which can lead to emittance transfer from one plane to the other and might lead to losses if the beam fills the aperture [21,22].

\subsubsection{Indirect Space Charge}

In the case of a beam off-axis in a perfectly conducting circular beam pipe (with radius $b$ ), a coherent (or dipolar, i.e. of the centre of mass) force arises, which can be found by using the method of the images (to satisfy the boundary condition on a perfect conductor, i.e. of a vanishing tangential electrical field). The electric field is always assumed to be non-penetrating. However, for the magnetic field, the situation is more complicated as it may or may not penetrate the vacuum chamber: the highfrequency components, called "ac" will not penetrate, while the low-frequency ones, called "dc" will penetrate and form images on the magnet pole faces (if there are some; otherwise they will go to infinity and will not act back on the beam). In the case of a non-penetrating "ac" magnetic field, one finally obtains (keeping only the linear terms, i.e. $\bar{x} \ll b$ and $\bar{y} \ll b$, where $\bar{x}$ and $\bar{y}$ are the transverse displacements 
of the centre of mass):

$$
F_{x}=\Lambda_{c} \bar{x}, \quad F_{y}=\Lambda_{c} \bar{y}, \quad \Lambda_{c}=\frac{\lambda e}{2 \pi \varepsilon_{0} \gamma^{2} b^{2}} .
$$

It can be seen that the transverse coherent space charge force of Eq. (4.6) is similar to the transverse incoherent space charge force of Eq. (4.4, left): $2 \sigma^{2}$ has been replaced by $b^{2}$.

The same analysis can be performed in the case of two infinite (horizontal) parallel plates spaced by $2 h$ and the results are the following (assuming that the transverse beam sizes are much smaller than $h$, assuming only the "ac" magnetic part and keeping only the linear terms)

$$
F_{x}=\Lambda_{c}\left(\frac{\pi^{2}}{24} \bar{x}-\frac{\pi^{2}}{24} x\right), \quad F_{y}=\Lambda_{c}\left(\frac{\pi^{2}}{12} \bar{y}+\frac{\pi^{2}}{24} y\right) .
$$

Therefore, compared to the circular case, the coherent force is smaller by $\pi^{2} / 24 \approx 0.4$ in the horizontal plane and $\pi^{2} / 12 \approx 0.8$ in the vertical one. Furthermore, there is a second incoherent (or quadrupolar, as it is linear with the particle position) term with opposite sign in both planes. The coefficients are linked to the Laslett coefficients usually used in the literature [23], and they are the same as the ones obtained by Yokoya [24] in the case of a resistive beam pipe under some assumptions (see Sect. 4.2). General formulae exist for the "real" tune shifts of coasting or bunched beams in pipes with different geometries, considering both the "ac" and "dc" magnetic parts and can be found for instance in Refs. [6, 7].

\subsection{Wake Fields and Impedances}

\section{E. Metral}

If the wall of the beam pipe is perfectly conducting and smooth, as it was the case in the previous section, a ring of negative charges is formed on the walls of the beam pipe where the electric field ends, and these induced charges travel at the same pace with the particles, creating the so-called "image" (or induced) current, which leads to real tune shifts. However, if the wall of the beam pipe is not perfectly conducting or contains discontinuities, the movement of the induced charges will be slowed down, thus leaving electromagnetic fields (which are proportional to the beam intensity) mainly behind: this is why these electromagnetic fields are called wake fields. The latter will create complex tune shifts leading to instabilities (see Sect. 4.3). What needs to be computed are the wake fields at the distance $z=s-v t$ behind the source particle (which is at position $s_{\text {source }}=v t$; with this convention, one has $z<0$ ) and their effects on the test or witness particles that compose the 
beam. The computation of these wake fields is quite involved and two fundamental approximations are introduced:

1. The rigid-beam approximation: The beam traverses a piece of equipment rigidly, i.e. the wake field perturbation does not affect the motion of the beam during the traversal of the impedance. The distance $z$ of the test particle behind some source particle does not change.

2. The impulse approximation: As the test particle moves at the fixed velocity $v=$ $\beta c$ through a piece of equipment, the important quantity is the impulse (and not the force) given by

$$
\Delta \boldsymbol{p}(x, y, z)=\int_{-\infty}^{+\infty} d t \boldsymbol{F}(x, y, s=z+\beta c t, t)=\int_{-\infty}^{+\infty} d t e(\boldsymbol{E}+\boldsymbol{v} \times \boldsymbol{B})
$$

where vectors are designated by boldtype letters. Starting from the four Maxwell equations for a particle in the beam, it can be shown that for a constant $\beta$ (which does not need to be 1) [9]

$$
\nabla \times \Delta \boldsymbol{p}(x, y, z)=0
$$

which is known as Panofsky-Wenzel theorem. This relation is very general, as no boundary conditions have been imposed. Only the two fundamental approximations have been made. Another important relation can be obtained when $\beta=1$, taking the divergence of the impulse, which is

$$
\nabla_{\perp} \cdot \Delta \boldsymbol{p}_{\perp}=0
$$

Considering the case of a cylindrically symmetric chamber (using the cylindrical coordinates $r, \theta, z$ ), yields the following three equations from Panofsky-Wenzel theorem

$$
\frac{1}{r} \frac{\partial \Delta p_{z}}{\partial \theta}=\frac{\partial \Delta p_{\theta}}{\partial z}, \quad \frac{\partial \Delta p_{r}}{\partial z}=\frac{\partial \Delta p_{z}}{\partial r}, \quad \frac{\partial\left(r \Delta p_{\theta}\right)}{\partial r}=\frac{\partial \Delta p_{r}}{\partial \theta} .
$$

The fourth relation when $\beta=1$ writes

$$
\frac{\partial\left(r \Delta p_{r}\right)}{\partial r}=-\frac{\partial \Delta p_{\theta}}{\partial \theta}
$$

Consider now as a source charge density a macro-particle of charge $Q=N_{b} e$ moving along the pipe (in the $s$-direction) with an offset $r=a$ in the $\vartheta=0$ direction 
and with velocity $v=\beta c$

$$
\begin{aligned}
\rho(r, \vartheta, s ; t) & =\frac{Q}{a} \delta(r-a) \delta_{p}(\vartheta) \delta(s-v t) \\
& =\sum_{m=0}^{\infty} \frac{Q_{m} \cos (m \vartheta)}{\pi a^{m+1}\left(1+\delta_{m 0}\right)} \delta(r-a) \delta(s-v t)=\sum_{m=0}^{\infty} \rho_{m},
\end{aligned}
$$

with $\boldsymbol{J}_{m}=\rho_{m} \boldsymbol{v}$, where $Q_{m}=Q a^{m}$ and $\delta$ is the Dirac function. In this case the whole solution can be written as, for $m \geq 0$ and $\beta=1$ (with $q$ the charge of the test particle and $L$ the length of the structure)

$$
\begin{gathered}
v \Delta p_{s}(r, \theta, z)=\int_{0}^{L} F_{s} d s=-q Q a^{m} r^{m} \cos m \theta W_{m}^{\prime}(z) \\
v \Delta p_{r}(r, \theta, z)=\int_{0}^{L} F_{r} d s=-q Q a^{m} m r^{m-1} \cos m \theta W_{m}(z), \\
v \Delta p_{\theta}(r, \theta, z)=\int_{0}^{L} F_{\theta} d s=q Q a^{m} m r^{m-1} \sin m \theta W_{m}(z) .
\end{gathered}
$$

The function $W_{m}(z)$ is called the transverse wake function (whose unit is $\mathrm{VC}^{-1} m^{-2 m}$ ) and $W_{m}^{\prime}(z)$ is called the longitudinal wake function (whose unit is $\mathrm{VC}^{-1} m^{-2 m+1}$ ) of azimuthal mode $m$. They describe the shock response of the vacuum chamber environment to a $\delta$-function beam which carries a $m$ th moment. The integrals (on the left) are called wake potentials. The longitudinal wake function for $m=0$ and transverse wake function for $m=1$ are therefore given by

$$
\begin{gathered}
W_{0}^{\prime}(z)=-\frac{1}{q Q} \int_{0}^{L} F_{s} d s=-\frac{1}{Q} \int_{0}^{L} E_{s} d s, \\
W_{1}(z)=-\frac{1}{q Q a} \int_{0}^{L} F_{x} d s=-\frac{1}{Q a} \int_{0}^{L}\left(E_{x}-v B_{y}\right) d s .
\end{gathered}
$$

The Fourier transform of the wake function is called the impedance. The idea of representing the accelerator environment by an impedance was introduced by Vaccaro [25] and Sessler [26]. As the conductivity, permittivity and permeability of a material depend in general on frequency, it is usually better (or easier) to treat the problem in the frequency domain, i.e. compute the impedance instead of the wake function. It is also easier to treat the case $\beta \neq 1$. Then, an inverse Fourier transform is applied to obtain the wake function in the time domain. The different relations linking the wake functions and the impedances are given by (with $k=\omega / v, \omega=2 \pi f$ with $f$ the frequency, and $j$ the imaginary unit)

$$
\begin{aligned}
& Z_{m}^{\|}(\omega)=\int_{-\infty}^{\infty} W_{m}^{\prime}(z) \mathrm{e}^{j k z} \frac{d z}{v}=\int_{-\infty}^{\infty} W_{m}^{\prime}(t) \mathrm{e}^{j k s} \mathrm{e}^{-j \omega t} d t \\
& Z_{m}^{\perp}(\omega)=-j \int_{-\infty}^{\infty} W_{m}(z) \mathrm{e}^{j k z} \frac{d z}{v}=-j \int_{-\infty}^{\infty} W_{m}(t) \mathrm{e}^{j k s} \mathrm{e}^{-j \omega t} d t . \\
& W_{m}^{\prime}(z)=\frac{1}{2 \pi} \int_{-\infty}^{\infty} Z_{m}^{\|}(\omega) \mathrm{e}^{-j k z} d \omega=\frac{1}{2 \pi} \int_{-\infty}^{\infty} Z_{m}^{\|}(\omega) \mathrm{e}^{-j k s} \mathrm{e}^{j \omega t} d \omega, \\
& W_{m}(z)=\frac{j}{2 \pi} \int_{-\infty}^{\infty} Z_{m}^{\perp}(\omega) \mathrm{e}^{-j k z} d \omega=\frac{j}{2 \pi} \int_{-\infty}^{\infty} Z_{m}^{\perp}(\omega) \mathrm{e}^{-j k s} \mathrm{e}^{j \omega t} d \omega .
\end{aligned}
$$


The unit of the longitudinal impedance $Z_{m}^{\|}(\omega)$ is $\Omega m^{-2 m}$ while the unit of the transverse impedance $Z_{m}^{\perp}(\omega)$ is $\Omega m^{-2 m+1}$. Furthermore, two important properties of impedances can be derived. The first is a consequence of the fact that the wake function is real, which leads to

$$
\left[Z_{m}^{\|}(\omega)\right]^{*}=Z_{m}^{\|}(-\omega), \quad-\left[Z_{m}^{\perp}(\omega)\right]^{*}=Z_{m}^{\perp}(-\omega),
$$

where * stands for the complex conjugate. The second is a consequence of PanofskyWenzel theorem

$$
Z_{m}^{\|}(\omega)=k Z_{m}^{\perp}(\omega)
$$

Another interesting property of the impedances is the directional symmetry (Lorentz reciprocity theorem): the same impedance is obtained from both sides if the entrance and exit are the same.

A more general definition of the impedances (still for a cylindrically symmetric structure) is the following

$$
Z_{m}^{\|}(\omega)=-\frac{1}{Q_{m}^{2}} \int d V E_{m}^{\|} J_{m}^{*}, \quad Z_{m}^{\perp}(\omega)=-\frac{1}{k Q_{m}^{2}} \int d V E_{m}^{\|} J_{m}^{*},
$$

where $d V=r d r d \vartheta d s$. For the previous ring-shape source it yields

$$
\begin{gathered}
Z_{0}^{\|}(\omega)=-\frac{1}{Q_{0}} \int_{0}^{L} d s E_{s}(r=a) \mathrm{e}^{j k s}, \\
Z_{1}^{\perp}(\omega)=-\frac{L}{k \pi a Q_{1}} \int_{0}^{2 \pi} d \vartheta E_{s}(r=a, \vartheta, s) \cos \vartheta \mathrm{e}^{j k s} .
\end{gathered}
$$

The situation is more involved in the case of non axi-symmetric structures (due in particular to the presence of the quadrupolar wake field, already discussed in Sect. 4.1) and for $\beta \neq 1$, as in this case some electromagnetic fields also appear in front of the source particle. In the case of axi-symmetric structures, a current density with some azimuthal Fourier component creates electromagnetic fields with the same azimuthal Fourier component. In the case of non axi-symmetric structures, a generalized notion of impedances was introduced by Tsutsui [27], where a current density with some azimuthal Fourier component may create an electromagnetic field with various different azimuthal Fourier components. If the source particle 1 and test particle 2 have the same charge $q$, and in the ultra-relativistic case, the transverse wake potentials can be written (taking into account only the linear terms with respect to the source and test particles and neglecting the constant, coupling and high order terms) [28]

$$
\begin{aligned}
& \int_{0}^{L} F_{x} d s=-q^{2}\left[x_{1} W_{x}^{\text {driving }}(z)-x_{2} W^{\text {detuning }}(z)\right], \\
& \int_{0}^{L} F_{y} d s=-q^{2}\left[y_{1} W_{y}^{\text {driving }}(z)+y_{2} W^{\text {detuning }}(z)\right],
\end{aligned}
$$


where the driving term is used here instead of dipolar and detuning instead of quadrupolar (or incoherent). In the frequency domain, Eq. (4.21) leads to the following generalized impedances

$$
\begin{aligned}
& Z_{x}[\Omega]=x_{1} Z_{x}^{\text {driving }}-x_{2} Z^{\text {detuning }} \\
& Z_{y}[\Omega]=y_{1} Z_{x}^{\text {driving }}+y_{2} Z^{\text {detuning }} .
\end{aligned}
$$

Note that in the case $\beta \neq 1$, another quadrupolar term is found [29].

From Eqs. (4.21) and (4.22), the procedure to simulate or measure the driving and detuning contributions can be deduced. In the time domain, using some time-domain electromagnetic codes like for instance CST Particle Studio [30], the driving and detuning contributions can be disentangled. A first simulation with $x_{2}=0$ gives the dipolar part while a second one with $x_{1}=0$ provides the quadrupolar part. It should be noted that if the simulation is done with $x_{1}=x_{2}$, only the sum of the dipolar and quadrupolar parts is obtained. The situation is more involved in the frequency domain, which is used for instance for impedance measurements on a bench [31]. Two measurement techniques can be used to disentangle the transverse driving and detuning impedances, which are both important for the beam dynamics (this can also be simulated with codes like Ansoft-HFSS [32]). The first uses two wires excited in opposite phase (to simulate a dipole), which yields the transverse driving impedance only. The second consists in measuring the longitudinal impedance, as a function of frequency, for different transverse offsets using a single displaced wire. The sum of the transverse driving and detuning impedances is then deduced applying the Panofsky-Wenzel theorem in the case of top/bottom and left/right symmetry [33]. Subtracting finally the transverse driving impedance from the sum of the transverse driving and detuning impedances obtained from the one-wire measurement yields the detuning impedance only. If there is no top/bottom or left/right symmetry the situation is more involved [34].

Both longitudinal and transverse resistive-wall impedances were already calculated 40 years ago by Laslett, Neil and Sessler [35]. However, a new physical regime was revealed by the CERN LHC collimators. A small aperture paired with a large wall thickness asks for a different physical picture of the transverse resistive-wall effect from the classical one. The first unstable betatron line in the LHC is around $8 \mathrm{kHz}$, where the skin depth for graphite (whose measured isotropic DC resistivity is $10 \mu \Omega \mathrm{m}$ ) is $1.8 \mathrm{~cm}$. It is smaller than the collimator thickness of $2.5 \mathrm{~cm}$. Hence one could think that the resistive thick-wall formula would be about right. In fact it is not. The resistive impedance is about two orders of magnitude lower at this frequency, as can be seen on Fig. 4.3. A number of papers have been published on this subject using the field matching technique starting from the Maxwell equations and assuming a circular geometry [36-40]. New results have been also obtained for flat chambers, extending the (constant) Yokoya factors to frequency and material dependent ones [41], as was already found with some simplified kicker impedance models $[42,43]$. Note that the material resistivity may vary with the magnetic field 


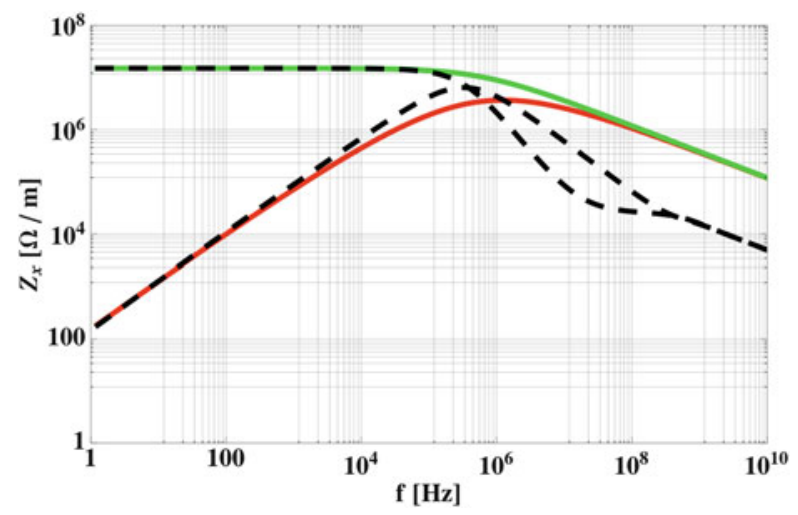

Fig. 4.3 Horizontal (driving) impedance of a cylindrical one-meter long LHC collimator (even if in reality the LHC collimators are composed of two parallel plates), with $b=2 \mathrm{~mm}$ and $\rho=10 \mu \Omega \mathrm{m}$. The real part is in red while the imaginary part is in green (note that in the classical thick-wall regime, the real and imaginary parts are equal). The dashed curves correspond to the case with a copper coating of $5 \mu \mathrm{m}$ [1]

through the magneto-resistance and the surface impedance can also increase due to the anomalous skin effect ([44] and references therein).

In the case of a cavity, an equivalent RLC circuit can be used with $R_{S}$ the longitudinal shunt impedance, $C$ the capacity and $L$ the inductance. In a real cavity, these three parameters cannot be separated easily and some other related parameters are used, which can be measured directly such as the resonance (angular) frequency $\omega_{r}=1 / \sqrt{L C}$, the quality factor $Q=R_{s} \sqrt{C / L}=R_{s} /\left(L \omega_{r}\right)=R_{s} C \omega_{r}$ and the damping rate $\alpha=\omega_{r} /(2 Q)$. When $Q=1$, the resonator impedance is called "broad-band", and this model was extensively used in the past in many analytical computations. The longitudinal and transverse impedances and wake functions (with $R_{\perp}$ the transverse shunt impedance) are given by [45] (see Fig. 4.4):

$$
\begin{gathered}
Z_{m}^{\|}(\omega)=\frac{R_{s}}{1+j Q\left(\frac{\omega}{\omega_{r}}-\frac{\omega_{r}}{\omega}\right)}, W_{m}^{\|}(t)=\frac{\omega_{r} R_{s}}{Q} \mathrm{e}^{-\alpha t}\left[\cos \left(\bar{\omega}_{r} t\right)-\frac{\alpha}{\bar{\omega}_{r}} \sin \left(\bar{\omega}_{r} t\right)\right] \\
Z_{m}^{\perp}(\omega)=\frac{\omega_{r}}{\omega} \frac{R_{\perp}}{1+j Q\left(\frac{\omega}{\omega r}-\frac{\omega_{r}}{\omega}\right)}, W_{m}^{\perp}(t)=\frac{\omega_{r}^{2} R_{\perp}}{Q \bar{\omega}_{r}} \mathrm{e}^{-\alpha t} \sin \left(\bar{\omega}_{r} t\right), \bar{\omega}_{r}=\omega_{r} \sqrt{1-\frac{1}{4 Q^{2}}} .
\end{gathered}
$$

Finally, all the transverse impedances (dipolar or driving and quadrupolar or detuning) should be weighted by the betatron function at the location of the impedances, as this is what matters for the effect on the beam, i.e. for the beam dynamics. Furthermore, all the weighted impedances can be summed and lumped at a single location around the ring (as the betatron phase advance does not play a role [46]) such that the transverse impedance-induced instabilities can be studied by considering only one interaction per turn, as it was confirmed in the past by performing macro-particle tracking simulations and comparing the cases of 

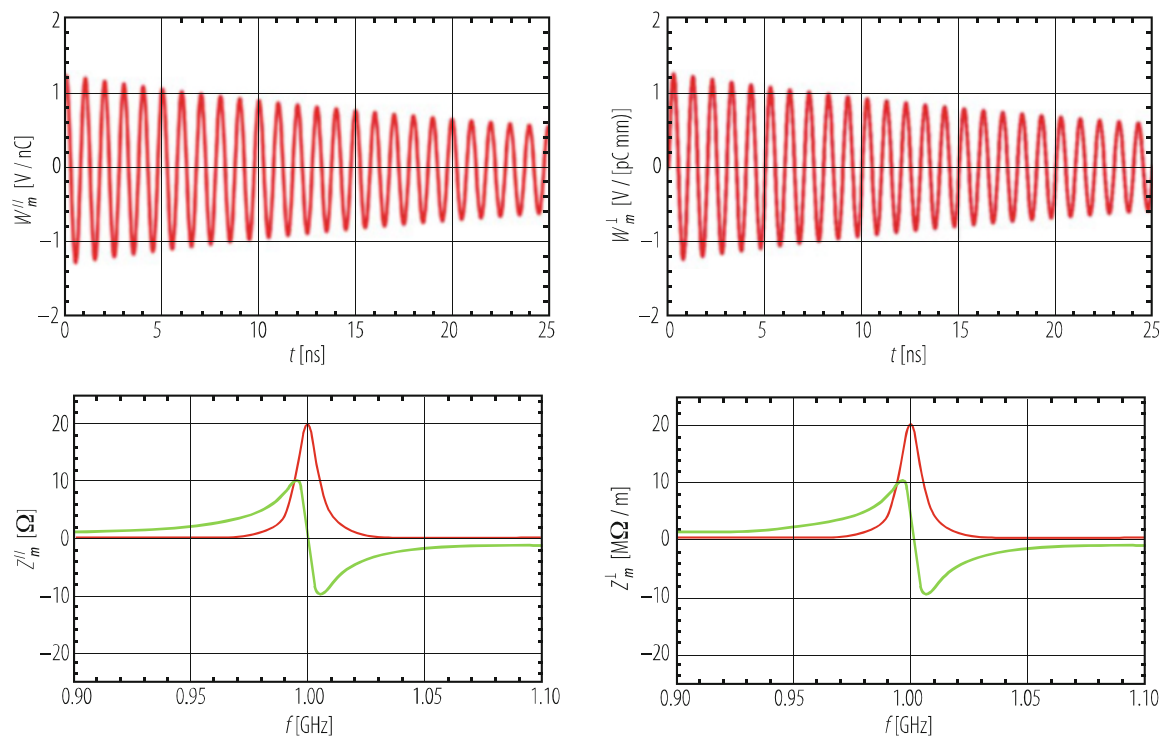

Fig. 4.4 Longitudinal and transverse impedances and wake functions, in the case of resonator impedances $\left(f_{r}=1 \mathrm{GHz}, Q=100, R_{\mathrm{S}}=20 \Omega\right.$, and $\left.R_{\perp}=20 \mathrm{M} \Omega / \mathrm{m}\right)$

distributed kickers in the CERN SPS with the corresponding lumped impedance: exactly the same result was obtained [47].

\subsection{Coherent Instabilities}

\section{E. Metral}

The wake fields can influence the motion of trailing particles, in the longitudinal and in one or both transverse directions, leading to energy loss, beam instabilities, or producing undesirable secondary effects such as excessive heating of sensitive components at or near the chamber wall. Therefore, in practice the elements of the vacuum chamber should be designed to minimise the self-generated electromagnetic fields. For example, chambers with different cross-sections should be connected with tapered transitions; bellows need to be separated from the beam by shielding; plates should be grounded or terminated to avoid reflections; high-resistivity materials should be coated with a thin layer of very good conductor (such as copper) when possible; etc.

Two approaches are usually used to deal with collective instabilities. One starts from the single-particle equation while the other solves the Vlasov equation, which is nothing else but an expression for the Liouville conservation of phase-space density seen by a stationary observer. In the second approach, the motion of the 
beam is described by a superposition of modes, rather than a collection of individual particles. The detailed methods of analysis in the two approaches are different, the particle representation is usually conveniently treated in the time domain, while in the mode representation the frequency domain is more convenient, but in principle they necessarily give the same final results. The advantage of the mode representation is that it offers a formalism that can be used systematically to treat the instability problem.

The first formalism was used by Courant and Sessler to describe the transverse coupled-bunch instabilities [48]. In most accelerators, the RF acceleration mechanism generates an azimuthal non-uniformity of the particle density and consequently the work of Laslett, Neil and Sessler for continuous beams [35] is not applicable in the case of bunched beams. Courant and Sessler studied the case of rigid (point-like) bunches, i.e. bunches oscillating as rigid units, and they showed that the transverse electromagnetic coupling of bunches of particles with each other can lead (due to the imperfectly conducting vacuum chamber walls) to a coherent instability. The physical basis of the instability is that in a resistive vacuum tank, fields due to a particle decay only very slowly in time after the particle has left (this leads to a longrange interaction). The decay can be so slow that when a bunch returns after one (or more) revolutions it is subject to its own residual wake field which, depending upon its phase relative to the wake field, can lead to damped or anti-damped transverse motion. For $M$ equi-populated equi-spaced bunches, $M$ coupled-bunch mode numbers exist $(n=0,1, \ldots, M-1)$, characterized by the integer number of waves of the coherent motion around the ring. Therefore the coupled-bunch mode number resembles the azimuthal mode number for coasting beams, except that for coasting beams there is an infinite number of modes. The bunch-to-bunch phase shift $\Delta \phi$ is related to the coupled-bunch mode number $n$ by $\Delta \phi=2 \pi n / M$.

Pellegrini [49] and, independently, Sands [50, 51] then showed that short-range wake fields (i.e. fields that provide an interaction between the particles of a bunch but have a negligible effect on subsequent passages of the bunch or of other bunches in the beam) together with the internal circulation of the particles in a bunch can cause internal coherent modes within the bunch to become unstable. The important point here is that the betatron phase advance per unit of time (or betatron frequency) of a particle depends on its instantaneous momentum deviation (from the ideal momentum) in first order through the chromaticity and the slip factor. Considering a non-zero chromaticity couples the betatron and synchrotron motions, since the betatron frequency varies around a synchrotron orbit. The betatron phase varies linearly along the bunch (from the head) and attains its maximum value at the tail. The total betatron phase shift between head and tail is the physical origin of the head tail instability. The head and the tail of the bunch oscillate therefore with a phase difference, which reduces to rigid-bunch oscillations only in the limit of zero chromaticity. A new (within-bunch) mode number $m=\ldots,-1,0,1, \ldots$, also called head-tail (or azimuthal) mode number, was introduced. This mode describes the number of betatron wavelengths (with sign) per synchrotron period.

The work of Courant and Sessler, or Pellegrini and Sands, was done for particular impedances and oscillation modes. Using the Vlasov formalism, Sacherer unified 

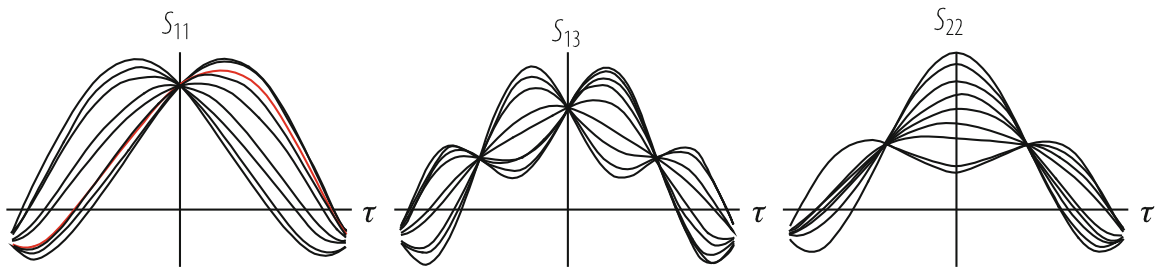

Fig. 4.5 Signal at the pick-up electrode for three different modes shown for several superimposed turns (the red line corresponds to one particular turn), for the case of the parabolic amplitude distribution and a constant inductive impedance (exhibiting therefore no growing oscillations!)

the two previous approaches, introducing a third mode number $q \equiv|m|+2 k$ (with $0 \leq k<+\infty$ ), called radial mode number, which comes from the distribution of synchrotron oscillation amplitudes $[52,53]$. It can be obtained by superimposing several traces of the directly observable average displacement along the bunch at a particular pick-up. The number of nodes is the mode number $q$ (see Figs. 4.5 and 4.8). The advantage of this formalism is that it is valid for generic impedances and any high order head-tail modes. This approach starts from a distribution of particles (split into two different parts, a stationary distribution and a perturbation), on which Liouville theorem is applied. After linearization of the Vlasov equation, one ends up with Sacherer's integral equation or Laclare's eigenvalue problem to be solved [53]. Because there are two degrees of freedom (phase and amplitude), the general solution is a twofold infinity of coherent modes of oscillation $(m, q)$. At sufficiently low intensity, only the most coherent mode (largest value for the coherent tune shift) is generally considered, leading to the classical Sacherer's formulae in both transverse and longitudinal planes. Note that contrary to the space charge case, these tune shifts are now complex, the imaginary part being linked to the instability growth rate. For protons a parabolic density distribution is generally assumed and the corresponding oscillation modes are sinusoidal (or close to it). For electrons, the distribution is usually Gaussian, and the oscillation modes are described in this case by Hermite polynomials. In reality, the oscillation modes depend both on the distribution function and the impedance, and can only be found numerically by solving the (infinite) eigenvalue problem. However, the mode frequencies are usually not very sensitive to the accuracy of the eigenfunctions. Similar results are obtained for the longitudinal plane.

It is worth mentioning that the CERN ISR suffered from a beam instability brought about by beams having different revolution frequencies. They could be in the same vacuum chamber or coupled by the beam-beam effect. The name of "overlap knock-out" [54] has been given to this phenomenon by which the stack is subjected to transverse kicks from the bunches. This produces blow-up of the stacked beam when the longitudinal frequency spectrum of the bunches overlaps with the betatron frequency spectrum of the coasting stacked beam. Similar problems limit the energy range of RHIC and proton lead in LHC [55]. 


\subsubsection{Longitudinal}

The most fundamental longitudinal instability encountered in circular accelerators is called the Robinson instability. The (Radio-Frequency) RF frequency accelerating cavities in a circular accelerator are tuned so that the resonant frequency of the fundamental mode is very close to an integral multiple of the revolution frequency of the beam. This necessarily means that the wake field excited by the beam in the cavities contains a major frequency component near a multiple of the revolution frequency. The exact value of the resonant frequency relative to the multiple of the revolution frequency is of critical importance for the stability of the beam. Above the transition energy, the beam will be unstable if the resonant frequency is slightly above it and stable if slightly below. This is the opposite below transition. This instability mechanism was first analyzed by Robinson [56]. Physically, the Robinson instability comes from the fact that the revolution frequency of an off-momentum beam is not given by the on-momentum revolution frequency, but by a quantity slightly different, depending on both the slip factor and the energy deviation.

Let's assume in the following that the Robinson criterion is met. A bunch is longitudinally stable if the longitudinal profile observed at a wall-current monitor is constant turn after turn and it is unstable if the longitudinal profile is not constant turn after turn. In the case of instability, the way the longitudinal profile oscillates gives some information about the type of instabilities. This was studied in detail by Laclare [53], who explained theoretically such pictures of "longitudinal (singlebunch) instability" starting from the single-particle longitudinal signal at a pickup electrode (assuming infinite bandwidth). The current signal induced by the test particle is a series of impulses delivered on each passage

$$
s_{z}(t, \vartheta)=e \sum_{k=-\infty}^{k=+\infty} \delta\left(t-\tau-\frac{\vartheta}{\Omega_{0}}-\frac{2 k \pi}{\Omega_{0}}\right),
$$

where $\tau$ is the time interval between the passage of the synchronous particle and the test particle, for a fixed observer at azimuthal position $\vartheta$ and $\Omega_{0}$ is the angular revolution frequency. In the frequency domain, the single-particle spectrum is therefore a line spectrum at (angular) frequencies $\omega_{p m}=p \Omega_{0}+m \omega_{s 0}$, where $\omega_{s 0}$ is the small-amplitude synchrotron frequency. Around every harmonic of the revolution frequency $p \Omega_{0}$, there is an infinite number of synchrotron satellites $m$ (it is different from the one used in Sect. 4.2!), whose spectral amplitude is given by the Bessel function $J_{m}\left(p \Omega_{0} \hat{\tau}\right)$, where $\hat{\tau}$ is the synchrotron amplitude. The spectrum is centred at the origin and because the argument of the Bessel functions is proportional to $\hat{\tau}$, the width of the spectrum behaves like $\hat{\tau}^{-1}$. Applying the Vlasov equation, linearizing it, and studying the effect of the impedance on the unperturbed distribution leads to the potential-well effect: a new fixed point is reached, with a new synchronous phase, a new effective voltage, a new synchrotron frequency, a new bunch length and a new momentum spread, which all depend on intensity. Studying a perturbation on top of the new stationary distribution, one ends up at low 
intensity, i.e. considering independently the modes $m$ (which is valid up to a certain intensity), with the following eigenvalue system

$$
\Delta \omega_{c m q} \sigma_{m q}(l)=\sum_{p=-\infty}^{p=+\infty} K_{l p}^{m} \sigma_{m q}(p), \text { with } \Delta \omega_{c m q}=\omega_{c m q}-m \omega_{s},
$$

where

$$
K_{l p}^{m}=-\frac{2 \pi I_{b} m \omega_{s}}{\Omega_{0}^{2} \hat{V}_{\mathrm{T}} h \cos \phi_{s}} j \frac{Z_{l}(p)}{p} \int_{\hat{\tau}=0}^{\hat{\tau}=+\infty} \frac{d g_{0}}{d \hat{\tau}} J_{m}\left(p \Omega_{0} \hat{\tau}\right) J_{m}\left(l \Omega_{0} \hat{\tau}\right) d \hat{\tau} .
$$

Here, $\Delta \omega_{c m q}$ is the coherent complex synchrotron frequency shift to be determined, $I_{b}=N_{b} e \Omega_{0} / 2 \pi$ is the bunch current, $\omega_{s}, \hat{V}_{\mathrm{T}}$ and $\phi_{s}$ are the new synchrotron frequency, total voltage and synchronous phase (taking into account the potentialwell distortion), $Z_{l}=Z_{0}^{\|}$is the longitudinal impedance, $g_{0}$ is the longitudinal amplitude density function and $h$ the RF harmonic number. The procedure to obtain first order exact solutions, with realistic modes and a general interaction, thus consists of finding the eigenvalues and eigenvectors of the infinite complex matrix whose elements are given by Eq. (4.26). The result is an infinite number of modes $m q$ of oscillation. To each mode, one can associate a coherent frequency shift $\Delta \omega_{c m q}=\omega_{c m q}-m \omega_{s}$ (which is the $q$ th eigenvalue), a coherent spectrum $\sigma_{m q}(p)$ (which is the $q$ th eigenvector) and a perturbation distribution $g_{m q}(\hat{\tau})$. For numerical reasons, the matrix needs to be truncated, and thus only a finite frequency domain is explored. For the case of the parabolic amplitude distribution and a constant inductive impedance (which leads to real tune shifts only and therefore no instability), the signal at the pick-up electrode shown for several superimposed turns is depicted on Fig. 4.5. In the case of a complex impedance, the real part will lead in addition to a growing amplitude with an associated instability rise-time. The spectrum of mode $m q$ is peaked at $f_{q} \approx(q+1) /\left(2 \tau_{b}\right)$ and extends $\sim \pm \tau_{b}^{-1}$, where $\tau_{b}$ is the full bunch length (in second). It can be seen from Fig. 4.5 that there are $q$ nodes on these "standing-wave" patterns. The longitudinal signal at the pick-up electrode is given by

$$
\begin{gathered}
S_{m q}(t, \vartheta)=S_{z 0}(t, \vartheta)+\Delta S_{z m q}(t, \vartheta), \\
S_{z 0}(t, \vartheta)=2 \pi I_{b} \sum_{p=-\infty}^{p=+\infty} \sigma_{0}(p) \mathrm{e}^{j p \Omega_{0} t} \mathrm{e}^{-j p \vartheta}, \sigma_{0}(p)=\int_{\hat{\tau}=0}^{\hat{\tau}=+\infty} J_{0}\left(p \Omega_{0} \hat{\tau}\right) g_{0}(\hat{\tau}) \hat{\tau} d \hat{\tau},
\end{gathered}
$$

$$
\Delta S_{z m q}(t, \vartheta)=2 \pi I_{b} \sum_{p=-\infty}^{p=+\infty} \sigma_{m q}(p) \mathrm{e}^{j\left(p \Omega_{0}+m \omega_{s}+\Delta \omega_{c m q}\right) t} \mathrm{e}^{-j p \vartheta}
$$


Finding the eigenvalues and eigenvectors of a complex matrix by computer can be difficult in some cases, and a simple approximate formula for the eigenvalues is useful in practice to have a rough estimate. This is known as Sacherer's (longitudinal) formula [52]. Sacherer's formula is also valid for coupled-bunch instability with $M$ equally-populated equally-spaced bunches, assuming multi-bunch modes with only one type of internal motion. In the case of gaps between bunch trains, a time-domain approach is usually better suited.

As the bunch intensity increases, the different longitudinal modes can no longer be treated separately and the situation is more involved. In the longitudinal plane, the microwave instability for coasting beams is well understood. It leads to a stability diagram, which is a graphical representation of the solution of the dispersion relation (taking into account the momentum spread) depicting curves of constant growth rates, and especially a threshold contour in the complex plane of the driving impedance (see Sect. 4.4) [57]. When the real part of the driving impedance is much greater than the modulus of the imaginary part, a simple approximation, known as the Keil-Schnell (or circle) stability criterion, may be used to estimate the threshold curve [58]. For bunched beams, it has been proposed by Boussard to use the coasting-beam formalism with local values of bunch current and momentum spread [59]. A first approach to explain this instability, without coasting-beam approximations, has been suggested by Sacherer through Longitudinal ModeCoupling (LMC) [60]. The equivalence between LMC Instabilities (LMCI) and microwave instabilities has been pointed out by Sacherer and Laclare [53] in the case of broad-band driving resonator impedances, when the bunch length is much greater than the inverse of twice the resonance frequency. Furthermore, due to the potential well-distortion, a bunch is more stable below transition than above [53, 61, 62]. Typical pictures of LMCI are shown in Fig. 4.6 [63] and a comparison with macroparticle tracking simulations, which revealed a good agreement, is discussed in Ref. [64]. Experimentally, the most evident signature of the LMCI is the intensitydependent longitudinal beam emittance blow-up to remain just below the threshold [65], as revealed also by macroparticle tracking simulations (see Fig. 4.7 [64]).

\subsubsection{Transverse}

A similar analysis as the one done for the longitudinal plane can be done in the transverse plane $[53,63]$. Following the same procedure, the horizontal coherent oscillations (over several turns) of a "water-bag" bunch (i.e. with constant longitudinal amplitude density) interacting with a constant inductive impedance are shown in Fig. 4.8.

The main difference with the longitudinal plane is that there is no effect of the stationary distribution and the bunch spectrum is now centered at the chromatic frequency $f_{\xi}=Q_{x 0} f_{0} \xi / \eta$, where $f_{0}$ is the revolution frequency and $\xi$ is the relative chromaticity. The sign of the chromatic frequency is very important and to avoid the head-tail instability (of mode 0) it should be slightly positive, meaning that 

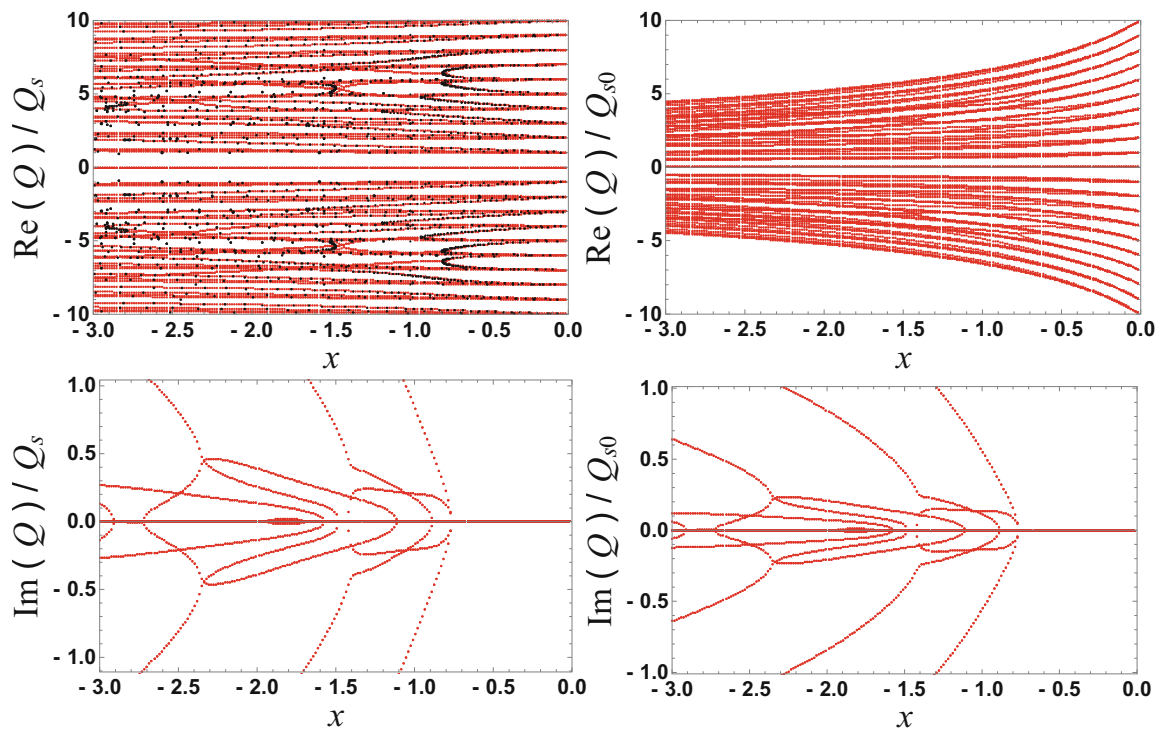

Fig. 4.6 (Left) Comparison between GALACLIC Vlasov Solver [63] (in red) and Laclare's approach [53] (in black) of the normalised mode-frequency shifts vs. the normalised parameter $x$ (proportional to the bunch intensity [63]), in the case of a broad-band resonator impedance (with a quality factor of 1 and a resonance frequency $f_{r}$ such that $f_{r} \tau_{b}=2.8$ ), above transition, without taking into account the potential-well distortion (this is why the intensity-dependent synchrotron tune $Q_{s}$ is used) and for a "Parabolic Amplitude Density" (PAD) longitudinal distribution [53]. (Right) Similar plot from GALACLIC only, taking into account the potential-well distortion (this is why the low-intensity synchrotron tune $Q_{s 0}$ is used)

the chromaticity should be negative below transition and positive above. Sacherer's formula is also valid for coupled-bunch instability with $M$ equally-populated equally-spaced bunches, assuming multi-bunch modes with only one type of internal motion (i.e. the same head-tail mode number). This analysis was extended in Ref. [66] to include also the coupling between the modes (and the possibility to have different head-tail modes in the different bunches). In the case of gaps between bunch trains, a time-domain approach is usually better suited.

At low intensity (i.e. below a certain intensity threshold), the standing-wave patterns (head-tail modes) are treated independently. This leads to instabilities where the head and the tail of the bunch exchange their roles (due to synchrotron oscillation) several times during the rise-time of the instability. The (approximate) complex transverse coherent betatron frequency shift of bunched-beam modes is given by Sacherer's formula for round pipes [52]. For flat chambers a quadrupolar effect (see Sect. 4.2) has to be added to obtain the real part of the coherent tune shift, which explains why the horizontal coherent tune shift is zero in horizontally flat chambers (of good conductors). As an example, a head-tail instability with mode $q=10$ is shown in Fig. 4.9(left). It is worth mentioning that there is also a head-tail instability in the longitudinal plane. The longitudinal head-tail instability, 

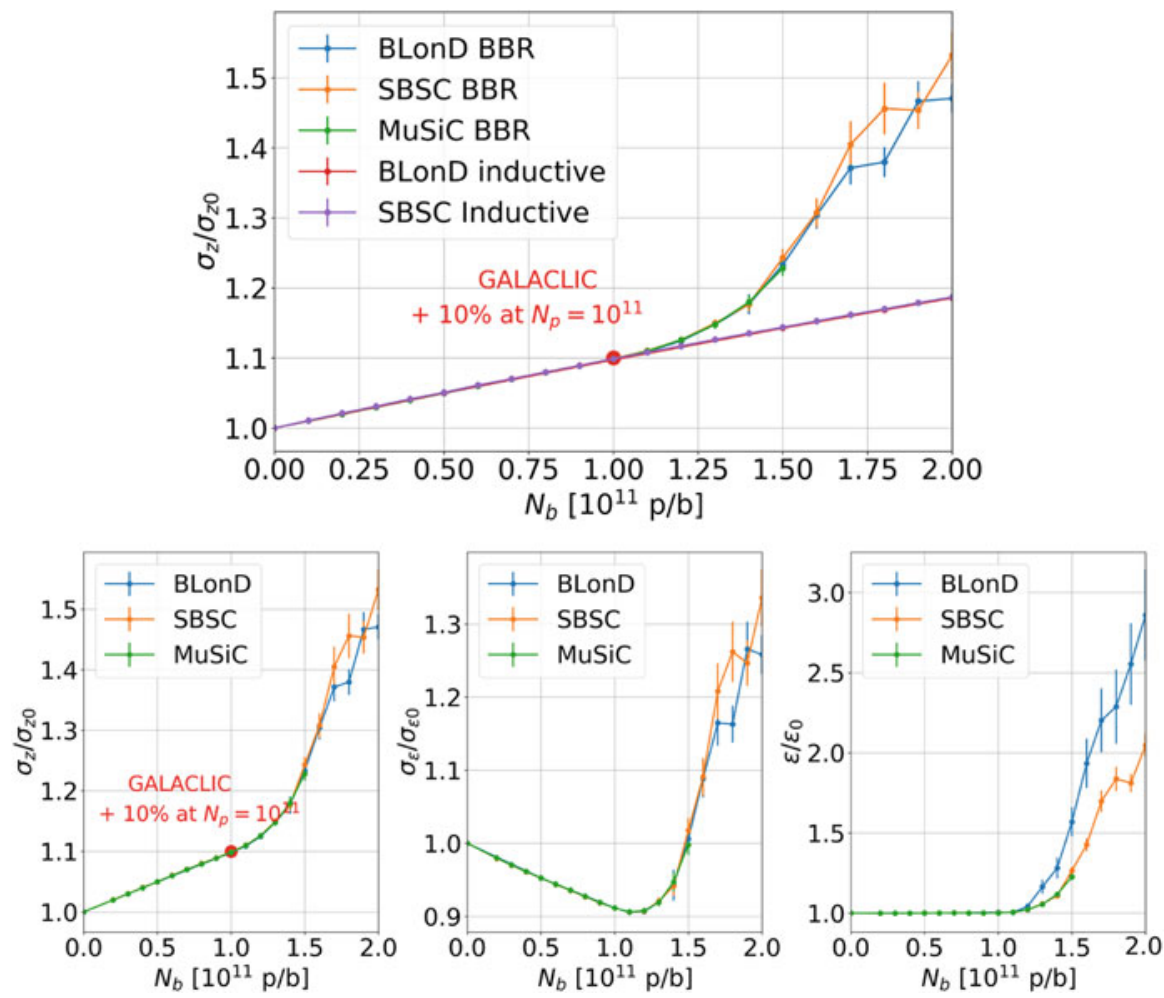

Fig. 4.7 Simulation results from BLonD, SBSC and MuSIC codes [64] corresponding to the case mentioned above: (upper) evolution of the normalised rms bunch length vs. bunch intensity for the cases of broad-band resonator and constant inductive impedances; (lower) evolution of the normalised rms bunch length, energy spread and longitudinal emittance vs. bunch intensity for the case of the broad-band resonator impedance. Courtesy of M. Migliorati [64]
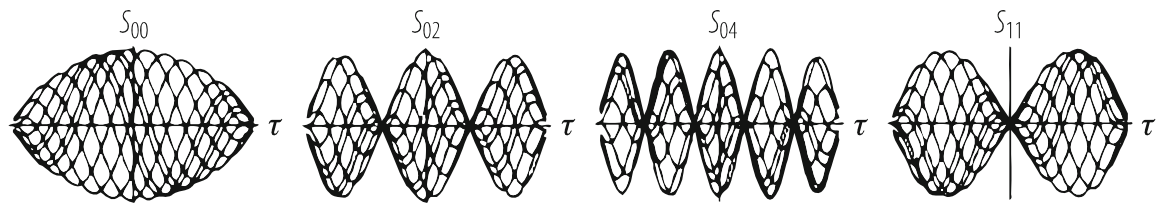

Fig. 4.8 Transverse signal at the pick-up electrode for four different modes shown for several superimposed turns, for the case of the "water-bag" bunch (i.e. with constant longitudinal amplitude density) and a constant inductive impedance. In the present example, the total phase shift between the head and the tail is given $\chi=\omega_{\xi} \tau_{b}=10$ (see below)

first suggested by Hereward [67] and possibly observed at the CERN SPS [68] results from the fact that the slip factor is not strictly a constant: it depends on the instantaneous energy error just as the betatron frequency does. The longitudinal beam distribution then acquires a head-tail phase, and instability may arise as a result. 

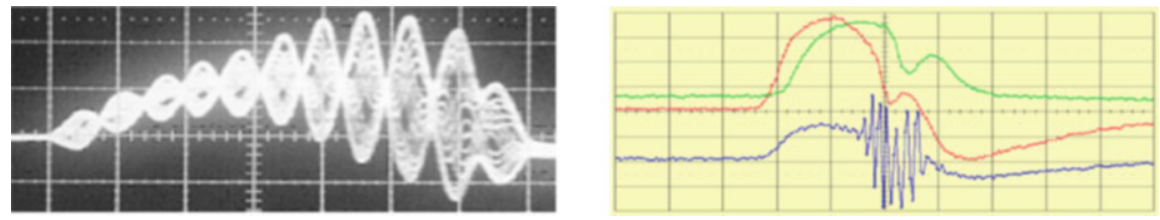

Fig. 4.9 (Left) Signal from a radial beam position monitor during 20 consecutive turns observed in the CERN PS at $1.4 \mathrm{GeV}$ kinetic energy in 1999. Time scale: $20 \mathrm{~ns} /$ div. (Right) Fast instability observed in the CERN PS near transition ( $\sim 6 \mathrm{GeV}$ total energy) in 2000. Single-turn signals from a wide-band pick-up. From top to bottom: $\sum, \Delta x$, and $\Delta y$. Time scale: $10 \mathrm{~ns} / \mathrm{div}$. The head of the bunch is stable and only the tail is unstable in the vertical plane. The particles lost at the tail of the bunch can be seen from the hollow in the bunch profile

As the bunch intensity increases, the different head-tail modes can no longer be treated separately. In this regime, the wake fields couple the modes together and a wave pattern travelling along the bunch is created: this is the Transverse Mode Coupling Instability (TMCI). The TMCI for circular accelerators has been first described by Kohaupt [69] in terms of coupling of Sacherer's head-tail modes. This extended to the transverse motion, the theory proposed by Sacherer to explain the longitudinal microwave instability through coupling of the longitudinal coherent bunch modes. The TMCI is the manifestation in synchrotrons of the Beam BreakUp (BBU) mechanism observed in linacs. The only difference comes from the synchrotron oscillation, which stabilises the beam in synchrotrons below a threshold intensity by swapping the head and the tail continuously. In fact, several analytical formalisms exist for fast (compared to the synchrotron period) instabilities, but the same formula is obtained (within a factor smaller than two) from five, seemingly diverse, formalisms in the case of a broad-band resonator impedance in the "longbunch" regime [70], as recently confirmed in Ref. [71]: (i) Coasting-beam approach with peak values, (ii) Fast blow-up, (iii) BBU (for 0 chromaticity), (iv) Post headtail, and (v) TMCI with 2 modes in the "long-bunch" regime (for 0 chromaticity). Two regimes are indeed possible for the TMCI according to whether the total bunch length is larger or smaller than the inverse of twice the resonance frequency of the impedance. The simple (approximate) formula reveals the scaling with the different parameters. In particular it can be seen that the instability does not disappear at high energy but saturates like the slip factor (what is important is not the energy but the distance from the transition energy) [72]. This means that the TMCI intensity threshold can be raised by changing the transition energy, i.e. by modifying the optics. Furthermore, the intensity threshold increases with the resonance frequency (as high-order head-tail modes will couple), with longitudinal emittance and with chromaticity. Note that the coherent synchrobetatron resonances, important in large machines, are not discussed here. This was checked with the MOSES Vlasov solver [73], which is a program computing the coherent bunched-beam modes. Below is a comparison between the MOSES code and the HEADTAIL code [74], which is a tracking code simulating single-bunch phenomena, in the case of a LHC-type single bunch at SPS injection [75]. As can be seen from Fig. 4.10, a very good 

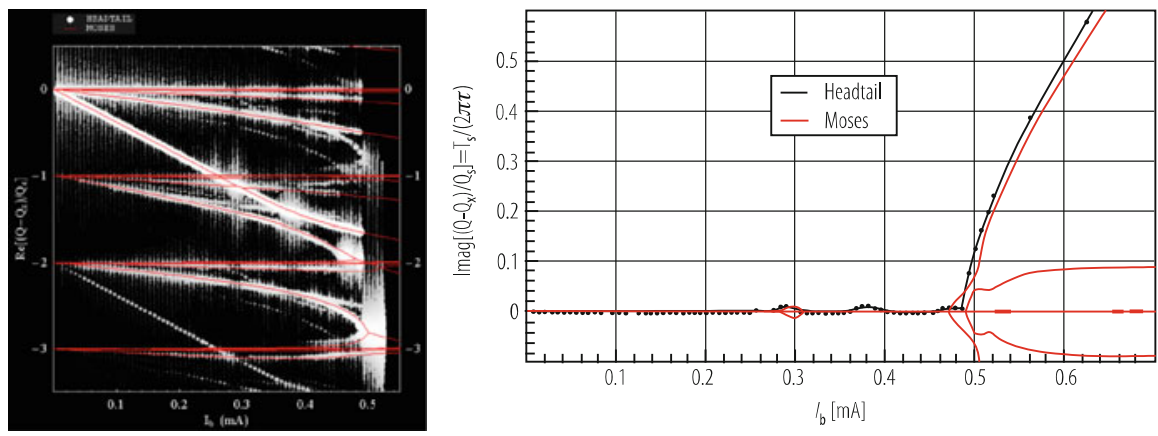

Fig. 4.10 Comparison between MOSES (in red) and HEADTAIL (in white) in the case of a broad-band resonator (Courtesy of Benoit Salvant [75]). Evolution of the real (left) and imaginary (right) parts of the shifts of the transverse modes (with respect to the unperturbed betatron tune), normalized by the synchrotron tune, vs. bunch intensity

agreement between the two was found. For a general impedance (i.e. not a resonator impedance) the situation is more involved and MOSES cannot be used: one should rely on HEADTAIL simulations or on the recently developed Vlasov solvers such as NHT [76] or DELPHI [77]. In the case of flat chambers, the intensity threshold is higher in one plane than in the other and linear coupling can be used to raise the TMCI intensity threshold [78]. Note finally that with many bunches the TMCI intensity threshold can be considerably reduced [66].

It is worth mentioning also all the work done for the TMCI in LEP, as Chin's work (with MOSES) came later. It was proposed to cure the TMCI with a reactive feedback that would prevent the zero mode frequency from changing with increasing beam intensity [79]. In [80, 81] a theory of reactive feedback has been developed in the two-particle approach and with the Vlasov equation. Theory has revealed that the reactive feedback can really appreciably increase the TMCI intensity threshold, which was confirmed by simulation [82, 83]. On the contrary, the resistive feedback was found to be "completely" ineffective as a cure for the TMCI [81]. An action of a feedback on the TMCI intensity threshold was later examined experimentally at PEP [84]. It was confirmed that a reactive feedback is indeed capable to increase the TMCI intensity threshold. But it turned out unexpectedly that a resistive feedback can also increase the TMCI intensity threshold and even more effectively [84]. In [85], an attempt was made to develop an advanced transverse feedback theory capable to elucidate the conditions at which the resistive or reactive or some intermediate feedback can cure the TMCI. Positive chromaticity above transition helps, but depending on the coupling impedance, beam stability may require a large value of the chromaticity either unattainable or which reduces the beam lifetime. It was proposed to have a negative chromaticity (what is usually avoided), where the zero mode is unstable (by head-tail instability) and all the other modes are damped, and stabilise this mode by a resistive feedback, keeping the higher order modes stable. In this case, the TMCI intensity threshold could be increased by a factor 3-5 [85]. In the last few years, several Vlasov solvers 

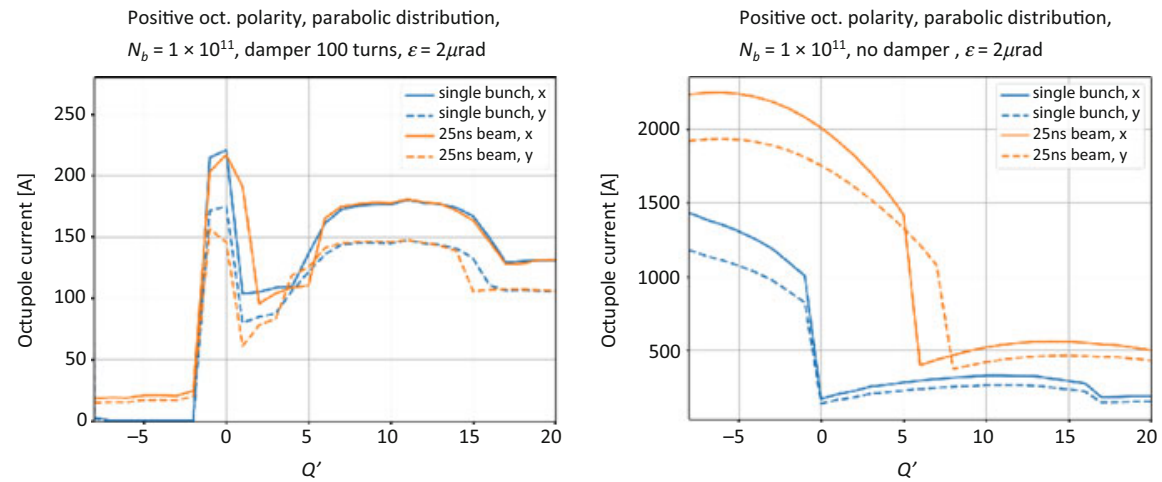

Fig. 4.11 Required Landau octupole current to stabilise the 2018 CERN LHC beam vs. chromaticity: (left) with resistive transverse damper and (right) without resistive transverse damper. Courtsey of N. Mounet [87]
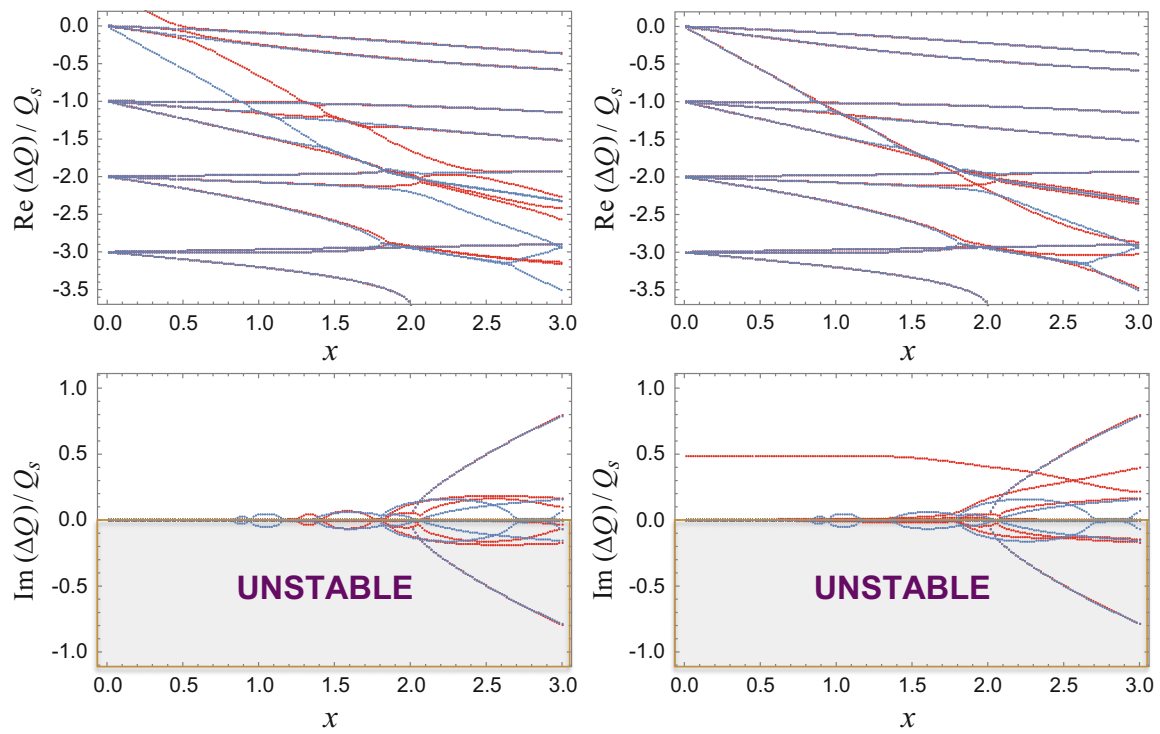

Fig. 4.12 Usual TMCI plots (for $f_{r} \tau_{b}=2.8$, i.e. in the "long-bunch" regime) showing the real and imaginary parts of the normalised complex tune shift vs. the normalised parameter $x$ (which is proportional to the bunch intensity [63]) without (in blue) and with (in red) a transverse damper: (left) reactive and (right) resistive [86]

were developed to take into account the effect of a transverse damper, such as NHT [76], DELPHI [77] and GALACTIC [86]. An example of DELPHI for the case of the LHC in 2018 is shown in Fig. 4.11, where the beneficial effect of the transverse resistive damper (on the required Landau octupole current needed to stabilise the beam) can be clearly seen. A comparison between a reactive and a resistive damper is shown in Figs. 4.12 and 4.13 using GALACTIC [86] (and a comparison between GALACTIC and Laclare's approach [53] is discussed in Ref. [63]). 

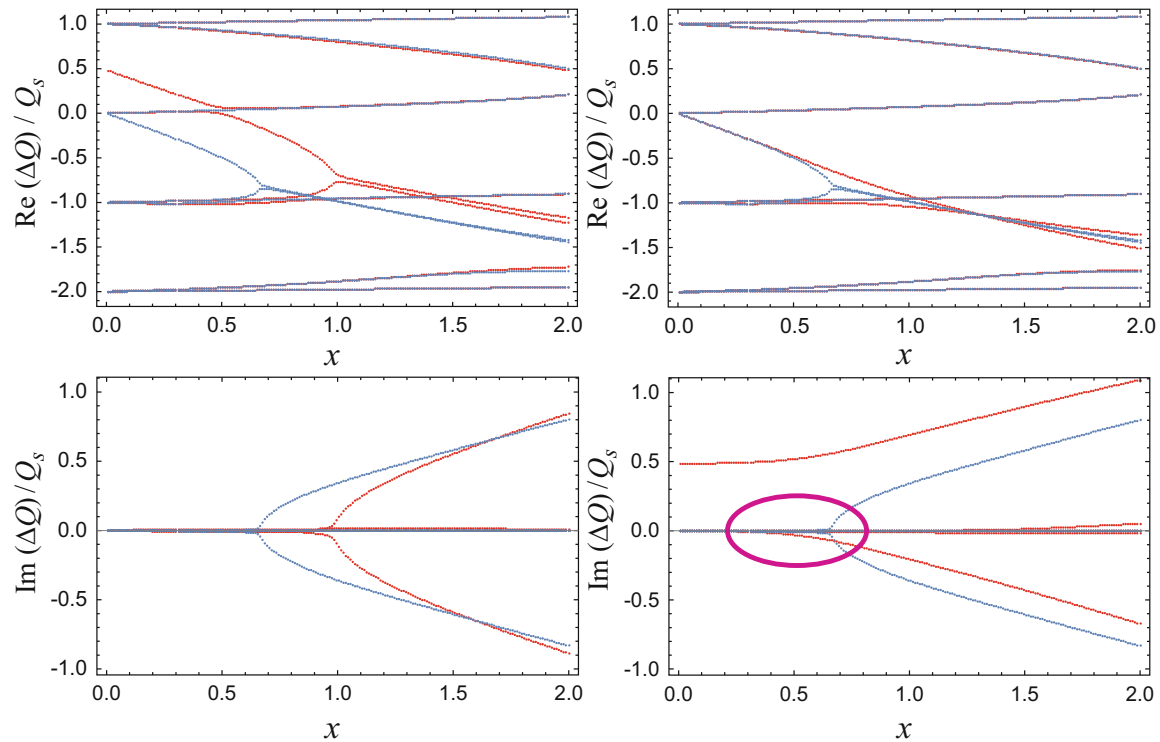

Fig. 4.13 Usual TMCI plots (for $f_{r} \tau_{b}=0.8$, i.e. in the "short-bunch" regime) showing the real and imaginary parts of the normalised complex tune shift vs. the normalised parameter $x$ (which is proportional to the bunch intensity [63]) without (in blue) and with (in red) a transverse damper: (left) reactive and (right) resistive [86]

As can be seen from Fig. 4.13(right) the resistive transverse damper exhibits a destabilising effect below the TMCI intensity threshold. This destabilising effect of (perfect) resistive transverse dampers was analysed in detail for the case of a single bunch with zero chromaticity [86]: in the presence of a resistive transverse damper the instability mechanism is completely modified as can be seen from Fig. 4.14. Due to the features, which are discussed in Ref. [86], the name "ISR (for Imaginary tune Split and Repulsion) instability" was suggested for this new kind of single-bunch instability with zero chromaticity.

It is also worth mentioning that in the case of hadrons (compared to leptons), another ingredient which should be taken into account while studying the transverse instabilities is space charge. This has been a subject of discussion for the last two decades as space charge was believed initially to be mainly beneficial as e.g. for the previous case of the CERN SPS TMCI predicted in the absence of space charge. It was recently found that space charge is actually destabilising in such a case ("longbunch" regime) [88-90], while it is beneficial in the "short-bunch" regime [88, 89]. This is clearly revealed in Figs. 4.15 and 4.16, but still some work is needed to fully understand what happens. 
Fig. 4.14 Usual TMCI plots showing the real and imaginary parts of the normalised complex tune shift vs. the normalised parameter $x$ (which is proportional to the bunch intensity [63]) without (in blue) and with (in red) a resistive transverse damper [86]
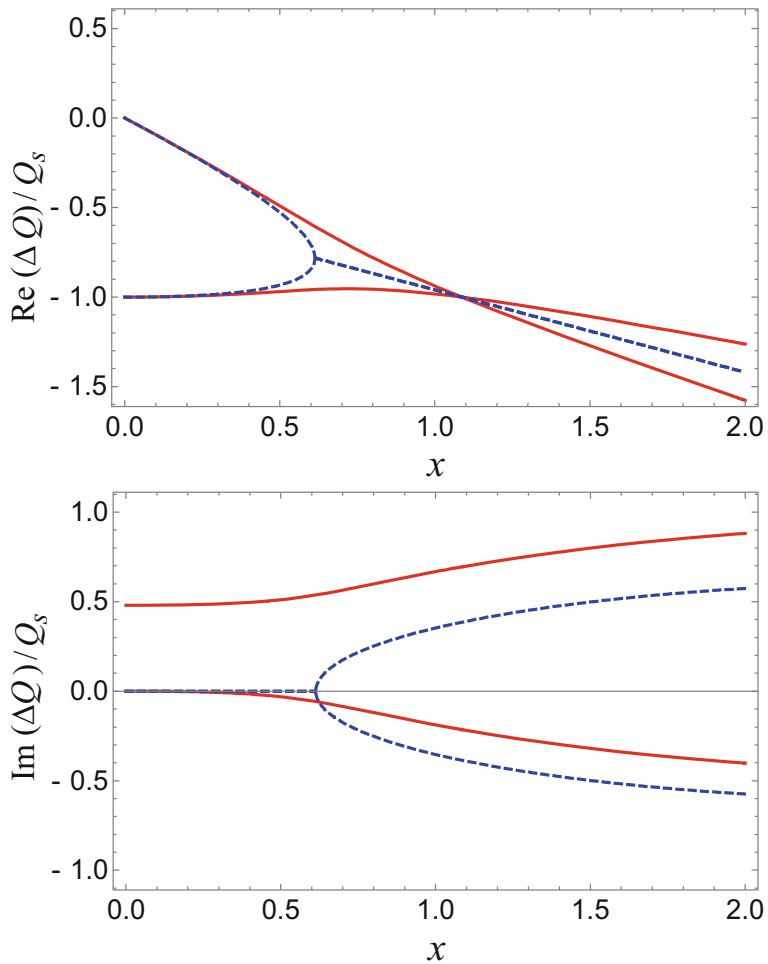

\subsection{Landau Damping}

\section{E. Metral}

Several stabilising mechanisms exist which can prevent the previous instabilities from developing. One of them is Landau damping, which is a general process that arises when one considers a whole collection of particles or other systems, which have a spectrum of resonant frequencies, and interact in some way. In accelerators we are usually concerned with an interaction of a kind that may make the beam unstable (wake fields), and we want to find out whether or not (and how) the spread of resonant frequencies will stabilise it. If the particles have a spread in their natural frequencies, the motion of the particles can lose its coherency. In order to understand the physical origin of this effect, let us first consider a simple harmonic oscillator, which oscillates in the $x$-direction with its natural frequency [8]. Let this oscillator be driven, starting at time $t=0$, by a sinusoidal force. The equation of motion is

$$
\ddot{x}+\omega_{x}^{2} x=f \cos \left(\omega_{c} t\right)
$$



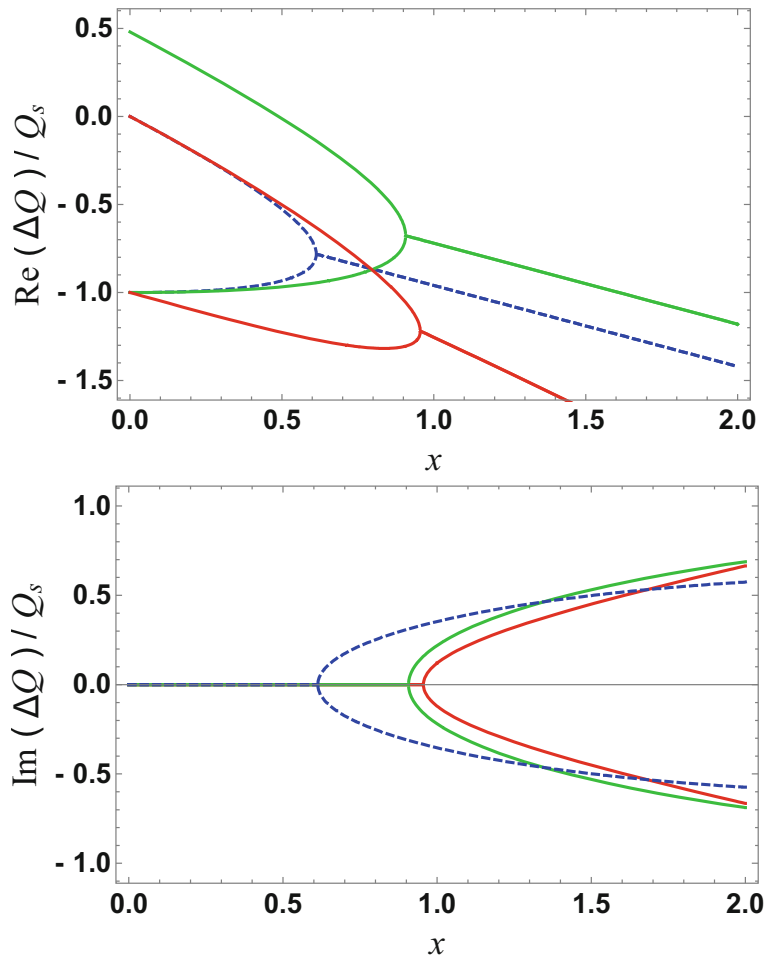

Fig. 4.15 Simplified model/example of Ref. [86], describing the mode-coupling in the "shortbunch" regime, i.e. the mode-coupling between modes 0 and -1 , extended here to take into account also space charge, using the parameters mentioned above: (dashed blue) with impedance only, (green) with impedance and a reactive transverse damper, (red) with impedance and space charge [88]. The normalised parameter $x$ is proportional to the bunch intensity [63]

where a dot stands for derivative with respect to time and with $x(0)=0$ and $\dot{x}(0)=$ 0 . The solution is

$$
x(t>0)=-\frac{f}{\omega_{c}^{2}-\omega_{x}^{2}}\left[\cos \left(\omega_{c} t\right)-\cos \left(\omega_{x} t\right)\right]=\frac{f}{2 \omega_{x 0}} \sin \left(\omega_{x 0} t\right) \frac{\sin \left[\left(\omega_{c}-\omega_{x}\right) t / 2\right]}{\left(\omega_{c}-\omega_{x}\right) / 2} .
$$

Consider now an ensemble of oscillators (each oscillator represents a single particle in the beam) which do not interact with each other and have a spectrum of natural frequency $\omega_{x}$ with a distribution $\rho_{x}\left(\omega_{x}\right)$ normalised to unity. Let's assume first that the origin of the betatron frequency spread is not specified: an externally given beam frequency spectrum is supposed. Now starting at time $t=0$, subject this ensemble of particles to the driving force $f \cos \left(\omega_{c} t\right)$ with all particles starting with initial conditions $x(0)=0$ and $\dot{x}(0)=0$. We are interested in the ensemble average 

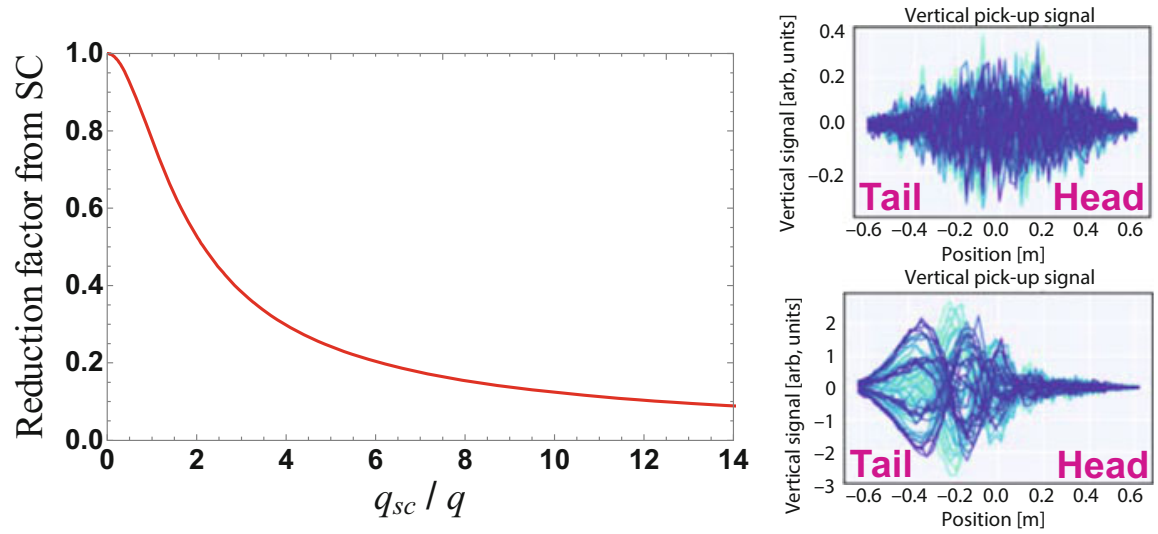

Fig. 4.16 (Left) reduction factor from a simplified model with space charge of the TMCI intensity threshold, as a function of the ratio between the space charge parameter $q_{s c}$ and the radial mode number $q$, in the case of the "long-bunch" regime, as e.g. for the CERN SPS at injection [88]. (Right) simulated stable bunch without space charge (top) and unstable bunch with space charge (bottom) for the case $q_{s c} l q=13.5$ with a bunch intensity a factor 3 lower than the TMCI intensity threshold without space charge [91] (Courtesy of A. Oeftiger)

of the response, which is given by superposition by

$$
\bar{x}(t)=\frac{f}{2 \omega_{x 0}}\left[\cos \left(\omega_{c} t\right) \mathrm{P} . \mathrm{V} . \int_{-\infty}^{+\infty} \frac{\rho_{x}\left(\omega_{x}\right)}{\omega_{x}-\omega_{c}} d \omega_{x}+\pi \rho_{x}\left(\omega_{c}\right) \sin \left(\omega_{c} t\right)\right],
$$

where P.V. stands for Principal Value. The sinus term has a definite sign relative to the driving force, because $\rho_{x}\left(\omega_{c}\right)$ is always positive. In particular, $\dot{\bar{x}}$ is always in phase with the force, indicating that work is being done on the system, which always reacts to the force "resistively". The Landau damping effect is to be distinguished from a "decoherence (also called phase-mixing, or filamentation) effect" that occurs when the beam has nonzero initial conditions. Had we included an initial offset, we would have introduced two additional terms into the ensemble response, which do not participate in the dynamic interaction of the beam particles and are not interesting for our purposes here. In this decoherence effect, individual particles continue to execute oscillations of constant amplitude, but the total beam response $\bar{x}$ decreases with time. As mentioned above, work is continuously being done on the system. However, the amplitude of $\bar{x}$, as given before, does not increase with time. Where did the energy go? The system absorbs energy from the driving force indefinitely while holding the ensemble beam response within bounds. The stored energy is incoherent in the sense that the energy is contained in the individual particles, but it is not to be regarded as heat in the system. This is because the stored energy is not distributed more or less uniformly in all particles, but is selectively stored in particles with continuously narrowing range of frequencies 
around the driving frequency. If one observes two particles, one with the exciting frequency $\omega_{c}$ and one with a frequency slightly different, at the beginning, they oscillate "coherently" (same amplitude and same phase). However, after a while the particle with the exciting frequency, being resonantly driven, continues to increase in amplitude as time increases, whereas the other particle with a slightly different frequency realizes that its frequency is not the same as the driving one and the "beating" phenomenon is observed for this particle. If one considers the phenomenon for a time $t$, the number of particles which still oscillate coherently decreases with time as $1 / t$, while their amplitude increases as $t$, the net contribution being constant with time.

The origins of the frequency spread that leads to Landau damping have not been taken into account till now. The case where the frequency spread comes from the longitudinal momentum spread of the beam is straightforward (for a coasting beam), because the longitudinal momentum is a constant, which just affects the coefficients in the equations of motion of the transverse oscillations, and hence their frequencies. It can be dealt with the same method as in the previous sections, i.e. it is the distribution function which is important. The same result applies also if one considers a tune spread that is due to a non-linearity (e.g. from octupole lenses) in the other plane. However, this result is no longer valid if the non-linearity is in the plane of coherent motion. In this case, the steady-state is more involved because the coherent motion is then a small addition to the large incoherent amplitudes that make the frequency spread, and it is inconsistent to assume that it can be treated as a linear superposition [92]. One needs to consider "second order" non-linear terms and the final result is that in this case it is not the distribution function which matters but its derivative. Using the Vlasov formalism, this result is recovered more straightforwardly.

\subsubsection{Transverse}

Considering the case of a beam having the same normalized rms beam size $\sigma=$ $\sqrt{\varepsilon}$ in both transverse planes, the Landau damping mechanism from octupoles of coherent instabilities, e.g. in the horizontal plane, is discussed from the following dispersion relation $[17,93]$

$$
1=-\Delta Q_{\mathrm{coh}}^{x} \int_{J_{x}=0}^{+\infty} d J_{x} \int_{J_{y=0}}^{+\infty} d J_{y} \frac{J_{x} \frac{\partial f\left(J_{x}, J_{y}\right)}{\partial J_{x}}}{Q_{c}-Q_{x}\left(J_{x}, J_{y}\right)-m Q_{s}},
$$

with

$$
Q_{x}\left(J_{x}, J_{y}\right)=Q_{0}+a_{0} J_{x}+b_{0} J_{y}
$$


Here, $Q_{c}$ is the coherent betatron tune to be determined, $J_{x, y}$ are the action variables in the horizontal and vertical plane respectively, with $f\left(J_{x}, J_{y}\right)$ the distribution function, $\Delta Q_{\mathrm{coh}}^{x}$ is the horizontal coherent tune shift, $Q_{x}\left(J_{x}, J_{y}\right)$ is the horizontal tune in the presence of octupoles, $m$ is the head-tail mode number, and $Q_{s}$ is the small-amplitude synchrotron tune (the longitudinal spread is neglected).

The $n$th order distribution function is assumed to be

$$
f\left(J_{x}, J_{y}\right)=a\left(1-\frac{J_{x}+J_{y}}{b}\right)^{n},
$$

where $a$ and $b$ are constants to be determined by normalization, and which corresponds to a profile extending up to $\sqrt{2(n+3)} \sigma$. The dispersion relation of Eq. (4.33) can be re-written as

$$
\Delta Q_{\mathrm{coh}}^{x}=-\frac{a_{0}}{n a b} I_{n}^{-1}(c, q),
$$

with

$$
\begin{gathered}
I_{n}(c, q)=\int_{J_{x}=0}^{1} d J_{x} \int_{J_{y}=0}^{1-J_{x}} d J_{y} \frac{J_{x}\left(1-J_{x}-J_{y}\right)^{n-1}}{q+J_{x}+c J_{y}}, \\
q=\frac{Q_{c}-Q_{0}-m Q_{s}}{-b a_{0}}, \text { and } c=\frac{b_{0}}{a_{0}} .
\end{gathered}
$$

It is convenient to write Eq. (4.36) in this way, with the left-hand-side (1.h.s) containing information about the beam intensity and the impedance and the righthand-side (r.h.s) containing information about the beam frequency spectrum only. In the absence of frequency spread, the r.h.s. of Eq. (4.36) is equal to $Q_{c}-Q_{0}-m Q_{s}$, which is thus given by $\Delta Q_{\text {coh }}^{x}$ (i.e. the 1.h.s). Calculation of the 1.h.s is now straightforward (following Sect. 4.3): for a given impedance (and transverse damper), one only needs to calculate the complex mode frequency shift, in the absence of Landau damping. Without frequency spread, the condition for the beam to be stable is thus simply $\operatorname{Im}\left(\Delta Q_{\mathrm{coh}}^{x}\right) \geq 0$ (oscillations of the form $\mathrm{e}^{j \omega t}$ are considered). Once its 1.h.s is obtained, Eq. (4.36) can be used to determine the coherent betatron tune $Q_{c}$ in the presence of Landau damping when the beam is at the edge of instability (i.e. $Q_{c}$ real). However, the exact value of $Q_{c}$ is not a very useful piece of information. The more useful question to ask is under what conditions the beam becomes unstable regardless of the exact value of $Q_{c}$ under these conditions, and Eq. (4.36) can be used in a reversed manner to address this question. To do so, one considers the real parameter $Q_{c}-Q_{0}-m Q_{s}$ (stability limit) and observes the locus traced out in the complex plane by the r.h.s of Eq. (4.36), as $Q_{c}-Q_{0}-m Q_{s}$ is scanned form $-\infty$ to $+\infty$. This locus defines a "stability boundary diagram". The 1.h.s of Eq. (4.36), a complex quantity, is then plotted in this plane as a single point. If this point lies on the locus, it means the solution of $Q_{c}$ for Eq. (4.36) is real, and this $Q_{c}-Q_{0}-m Q_{s}$ 



Fig. 4.17 Stability diagrams (for both positive and negative detunings $a_{0}$ ) for the LHC at top energy $(7 \mathrm{TeV})$ with maximum available octupole strength: (Left) for the 2nd order (dashed curves), the 15th order (full curves), and the Gaussian (dotted curves) distribution; (Right) for the Gaussian distribution (dotted curve) and a distribution with more populated tails than the Gaussian (full curve)

is such that the beam is just at the edge of instability. If it lies on the inside of the locus (the side which contains the origin), the beam is stable. If it lies on the outside of the locus, the beam is unstable. The stability diagrams for the 2 nd order, 15 th order and Gaussian distribution functions are plotted in Fig. 4.17 for the case of the LHC at top energy $(7 \mathrm{TeV})$ with maximum available octupole strength $(\varepsilon=0.5 \mathrm{~nm}$, $\left|a_{0}\right|=270440$ and $\left.c=-0.65\right)$.

The case of a distribution extending up to $6 \sigma$ (as the 15 th order distribution) but with more populated tails than the Gaussian distribution has also been considered and revealed a significant enhancement of the stable region compared to the Gaussian case ([93], see also Fig. 4.17(right)). This may be the case in reality in proton machines due to diffusive mechanisms.

It is worth reminding that Landau damping of coherent instabilities and maximization of the dynamic aperture are partly conflicting requirements. On the one hand, a spread of the betatron frequencies is needed for the stability of the beam coherent motion, which requires nonlinearities to be effective at small amplitude. On the other hand, the nonlinearities of the lattice must be minimized at large amplitude to guarantee the stability of the single-particle motion. A trade-off between Landau damping and dynamic aperture is therefore usually necessary [87].

Despite the destabilising effect of a resistive transverse damper in the case of a single bunch with zero chromaticity (as discussed in Sect. 4.3.2) below the TMCI intensity threshold (in the case of the "short-bunch" regime) without transverse damper, a transverse damper helps to reduce the amount of tune spread which would be needed to stabilise the bunch above the TMCI intensity threshold, as it can be seen in Fig. 4.18.

Note that linear coupling between the transverse planes can also influence the Landau damping mechanism [95], leading to a sharing of the Landau damping between the transverse planes, which can have a beneficial effect (i.e. stabilising the other plane, as it was used in the CERN PS for many years [96]) or a detrimental effect (i.e. destabilising one or two planes by loss of Landau damping, as it was 
Fig. 4.18 Required normalised (to the synchrotron tune $Q_{s}$ ) tune spread $\Delta q$ to stabilise the bunch in both cases of instabilities without and with Transverse Damper (TD), corresponding to the cases of Fig. 4.14 [94]. The normalised parameter $x$ is proportional to the bunch intensity [63]

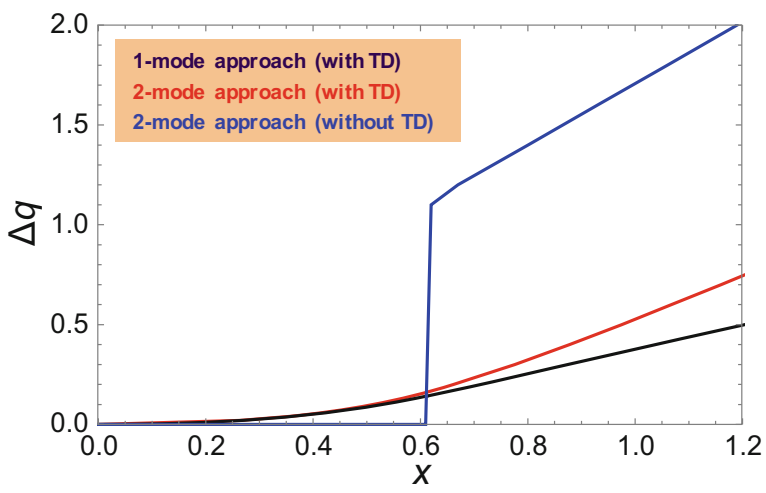

Fig. 4.19 Reduction of the tune footprint (and associated projections on the transverse tunes axes, responsible for Landau damping) vs. linear coupling (described here by the "closest tune approach" $\left|C^{-}\right|$) [98]. Courtesy of L.R. Carver

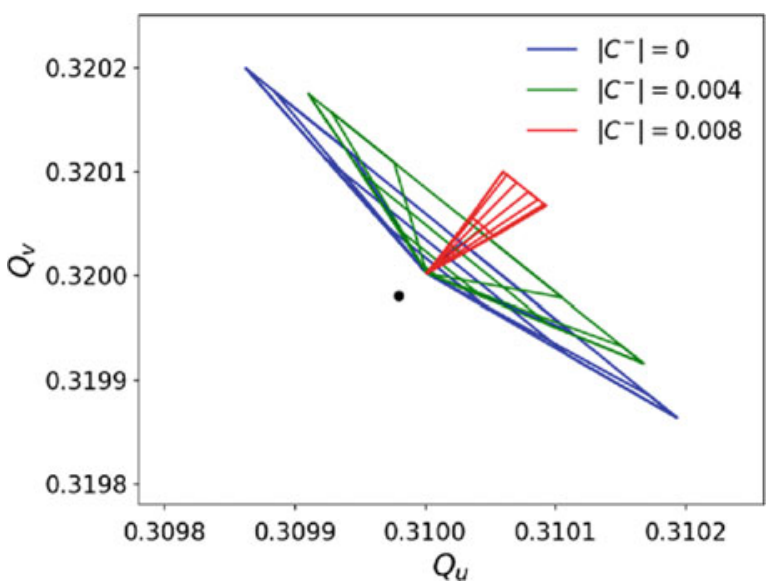

believed to be the case with the Batman instability of the HERA proton ring [97]: due to the features discussed in Ref. [97], the name "coupled head-tail instability" was suggested for this instability in the HERA proton ring). Recently, linear coupling was also observed to be detrimental in the CERN LHC [98], as revealed by both measurements and macroparticle simulations (see Fig. 4.19). This required a careful measurement and correction of linear coupling all along the LHC cycle to avoid to use much more Landau octupoles current than foreseen. One has also to remember that linear coupling modifies also the transverse emittances [99, 100].

In the case of additional space-charge nonlinearities, the stability diagram will be shifted and beam stability can be obtained or lost, depending on the coherent tune. The influence of space-charge nonlinearities on the Landau damping mechanism of transverse coherent instabilities has first been studied by Möhl and Schönauer for coasting and rigid bunched beams [101, 102]. It was studied in detail in the past years for higher-order head-tail modes from both theory [103-106] and numerical simulations [107]. 
The interplay between Landau octupoles and beam-beam long-range interactions can be either beneficial or detrimental depending on the sign of the Landau octupoles current (see Sect. 4.6) [108] and this effect has to be careful taken into account in the CERN LHC to be able to push its performance.

Another destabilising effect currently under investigation at the CERN LHC (and which could explain some long latencies observed in the past, of the order of few minutes or even tens of minutes) is the effect of noise, whose detrimental effect was predicted in 2012 [109] and confirmed experimentally in 2018 [110].

Some work is being done to try and use the nonlinear optics as a path to high intensity, providing "infinite (transverse) Landau damping" [111], or electron lenses [112] or Radio Frequency Quadrupoles (or similarly second order chromaticity) [113-115]. The latter two methods are believed to be more efficient than Landau octupoles at high energy due to the adiabatic damping and the associated significant reduction of the transverse beam sizes.

\subsubsection{Longitudinal}

When the bunch is very small inside the RF bucket, the motion of the particles is linear and all the particles have the same (unperturbed, maximum) synchrotron frequency $\omega_{s 0}$. By increasing the bunch length the incoherent synchrotron frequency spread $S$ is increased (the maximum synchrotron frequency spread is obtained when the bunch length is equal to the RF bucket length as in this case the synchrotron frequency of the particles with the largest amplitude is equal to 0: the synchrotron frequency spread $S$ is equal to $\omega_{s 0}$ in this case). In the presence of an impedance, the coherent synchrotron frequency of the dipole mode $\omega_{c 11}$, which is equal to the low-intensity synchrotron frequency $\omega_{s 0}$ without synchrotron frequency spread (due to the compensation between the incoherent and coherent tune shifts), moves closer and closer to the incoherent band (stable region). The two possible cases are represented in Fig. 4.20 (using the rigid-bunch approximation), which is similar to what was obtained by Besnier (who considered a parabolic distribution function, which introduces some pathologies in the stability diagram due to its sharp edge) ([116], and references therein): the case of a capacitive impedance below transition or inductive impedance above transition corresponds to $U>0$ (the coherent synchrotron frequency shift of the dipole mode has been written $\Delta \omega_{c 11}=U-j V$ ) and the incoherent synchrotron frequency shift (due to the potential-well distortion) is $\Delta \omega_{s}^{i}<0$ (and thus $\omega_{s}<\omega_{s 0}$ ), and the case of a capacitive impedance above transition or inductive impedance below transition corresponds to $U<0$ and $\Delta \omega_{s}^{i}>0$ (and thus $\left.\omega_{s}>\omega_{s 0}\right)$. Motions $\propto \mathrm{e}^{j \omega t}$ are considered, which means that the beam is unstable when $V>0$ ( $V$ is called the instability growth rate). The usual case where the resistive part of the impedance is small compared to the imaginary part is assumed, i.e. $V \ll|U|$. Beam stability is obtained when $\omega_{c 11}$ enters into the incoherent band. In both cases, the stability limit is reached for $k=4$, i.e. $S=4|U|$, which is Sacherer's stability criterion for the dipole mode. 
Fig. 4.20 Evolution of the coherent synchrotron frequency for the dipole mode with respect to the incoherent frequency spread (using the rigid-bunch approximation)

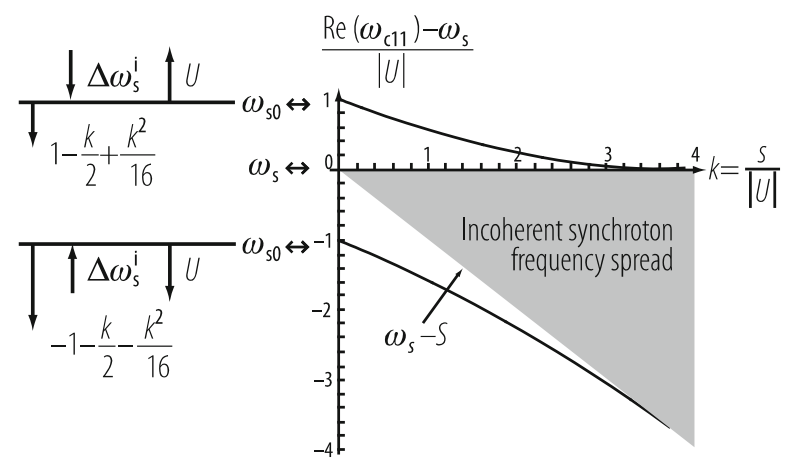

\title{
4.5 Two-Stream Effects (Electron Cloud and Ions)
}

\author{
G. Rumolo
}

\subsubsection{Electron Cloud Build-Up in Positron/Hadron Machines}

The term "electron cloud" is used for describing an accumulation of electrons inside the beam chamber of a circular accelerator, in which bunched beams of positively charged particles are accelerated or stored. The electron cloud can affect the accelerator operation by causing beam tune shift, emittance growth and coherent instabilities, as well as increase of the vacuum pressure and interference with beam diagnostics devices. Most of these effects eventually lead to beam quality degradation and loss. Electrons can be initially produced in the vacuum chamber by a number of processes. These electrons are called primary because, although some times their number can be sufficiently high to affect the circulating beam, they are usually only the seed for an avalanche process (see Fig. 4.21). In general, the primary electrons rapidly multiply via a beam-induced multipacting mechanism, which involves acceleration of the electrons in the beam field and secondary emission from their impact on the chamber wall. In the following, we first give an overview on the electron cloud formation (or build-up) process. We therefore list the main primary generation mechanisms and then discuss how the thus generated electrons can multipact in the presence of a train of bunches. The other important stages of the build-up of an electron cloud are its equilibrium and successive decay in the gap behind a bunch train. In the second part, we briefly discuss dynamics and consequences of the beam instabilities caused by the electron cloud that has formed in a beam pipe. In conclusion, we will try to give an up-to-date list of the possible techniques for electron cloud mitigation or suppression.

Primary electrons are the electrons generated during the passage of a bunch. They can be photo-electrons from synchrotron radiation in bending regions (mainly for positron beams) or secondary electrons desorbed from beam particles lost at the 


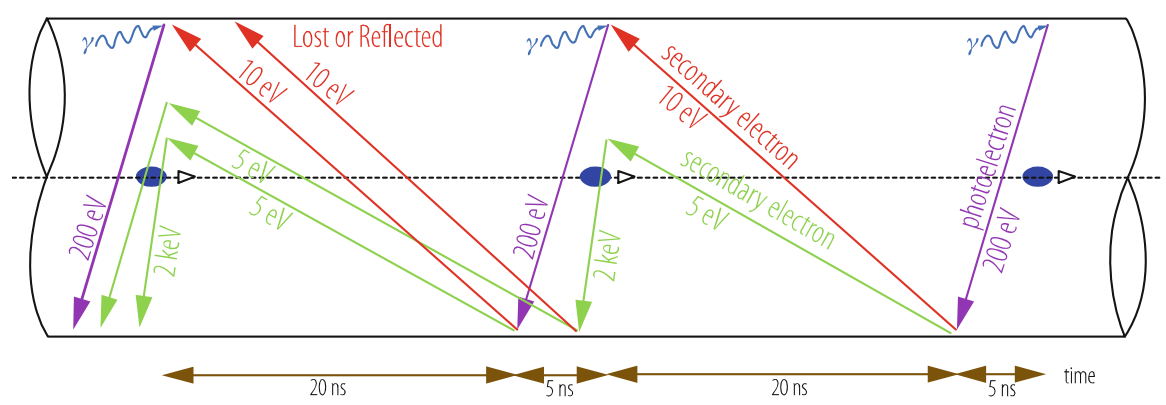

Fig. 4.21 Schematic of electron cloud build-up in the LHC beam pipe during multiple bunch passages, via photo-emission (due to synchrotron radiation) and secondary emission (Courtesy of F. Ruggiero). Note that the LHC is the 1st proton storage ring for which synchrotron radiation becomes a noticeable effect

walls (especially for ion beams). In this case they are emitted from the chamber wall. Primary electrons can also be created within the volume swept by the beam if the production mechanism is ionization of the residual gas. The location where the electrons are created can determine the energy gain of the primary electrons in the beam potential. The number of electrons created per unit length by synchrotron radiation or by beam loss during one bunch passage can be comparable to the average line density of beam particles, in which case these processes can alone give rise to amounts of electrons critical for the beam stability. The rate of photoemission (number of photoelectrons created per unit length) can be estimated as the product of the photo-electron yield $Y_{\gamma}$ by the photoemission rate $d N_{\gamma} / d s$ :

$$
\frac{d N_{e \gamma}}{d s}=Y_{\gamma} \frac{d N_{\gamma}}{d s}=Y_{\gamma} \frac{5 \alpha \gamma}{2 \sqrt{3} \rho},
$$

where $\alpha$ denotes the fine structure constant and $\rho$ the curvature radius of the beam in the dipole. For many materials, the photo-emission yield can be correctly approximated as being about 0.1 over a fairly large photon energy range, e.g. between a few $\mathrm{eV}$ and a few tens of $\mathrm{keV}$. The azimuthal distribution of absorbed photons around the chamber wall and, thus, the launch positions of the emitted primary photo-electrons depend on the reflective properties of the chamber wall. The first simulation of an electron cloud build-up for short bunches was written by K. Ohmi. It served to explain coupled-bunch instabilities observed with positron beams at the KEK photon factory [117]. Ohmi's pioneering study considered only photo-emission at the chamber wall as a source of electrons, though a little later his initial code was extended to include secondary emission by electrons as well.

Ionization from scattering of individual charged beam particles against molecules of the residual gas occurs with typical cross sections of 1-2 Mbarn for most of the gas species that can be found in a beam chamber. However, a lower cross section of about 0.2 Mbarn applies to the lighter species, like $\mathrm{H}_{2}$ [118]. These numbers 
refer to singly charged particles at ultrarelativistic energies. For fully ionized atoms, the cross section scales roughly with the square of the atomic number, i.e. like $Z^{2}$. Additionally, it increases by several orders of magnitude towards lower beam energies. If the beam density is sufficiently high, as it will be in certain sections of the next generation of linear colliders, ionization by the collective electric field of the bunch replaces single-particle scattering ionization as the dominant ionization process [119]. When this happens, the beam completely and instantly ionizes the residual gas in its neighbourhood.

Protons or ions impacting on the wall can be responsible for the generation of a large number of electrons. The secondary-electron yield from ion impact is approximately proportional to the projectile stopping power and inversely proportional to the cosine of the angle of incidence [120]. Since the stopping power is in turn proportional to the square of the charge number divided by the mass number, this value, usually very high because of the shallow angles at which losses typically occur, can be further amplified by one or two orders of magnitude for heavy ion beams. Production of this type of electrons also occurs with a high rate at the collimators, where significant beam loss routinely occurs by design.

The mechanism responsible for an exponential growth of the number of electrons is beam induced multipacting. The primary electrons are accelerated by the electric field of a passing bunch to such high energies that they produce, on average, more than one secondary electron when they again hit the wall of the vacuum chamber. The Secondary Emission Yield (SEY) of the chamber material is by definition the number of secondary electrons produced on average by an electron impact. It is obviously a function of the impinging electron energy, its angle of incidence, and the chamber history. For a round chamber of radius $h$ and a short bunch, the resonance condition for beam-induced multipacting from electrons produced at the pipe walls takes the simple form [121]

$$
N_{b} r_{e} L_{b}=h^{2}
$$

where $r_{e}$ denotes the classical electron radius and $L_{b}$ the bunch spacing in units of length. However, the condition of Eq. (4.40) is by far too stringent. Most secondary electrons have low energy and tend to stay in the vacuum chamber for a long time after a bunch passage. The survival of low energy electrons is made even more likely by the fact that their probability of being elastically backscattered at the chamber walls is almost one [122]. Moreover, a different multipacting regime, called "trailing edge multipacting", also exists in presence of long bunches. The electrons produced on the falling edge of the bunch can gain energy as they cross the pipe section and cause multipacting just during the passage of the second part of the bunch. This is a special type of single-bunch multipacting, causing electron clouds that can be either significantly cleared before the arrival of the next long bunch, or accumulate further in a mixed single-bunch and multi-bunch process.

Many simulation codes have been developed over the years to study numerically the process of electron cloud build-up and explore different beam/machine parameter ranges (e.g. [123-125]). Due to the variety of possible regimes and processes 
involved, simple considerations have usually turned out to be insufficient to predict the build-up thresholds and the change in electron cloud caused by some specific parameter change. It was a gratifying confirmation of the predictive power of the early simulations that the expected electron clouds were indeed observed at both B factories (PEP-II and KEKB), at the Large Hadron Collider injectors PS and SPS when operated with LHC-type beams, and finally in the LHC itself when operation with trains of closely spaced bunches started. In these rings, the electron cloud was seen to cause tune shift and emittance growth along the bunches of a train, both coupled and single-bunch instabilities and a degradation of certain beam diagnostics signals [126-128]. In the SPS and PS, significant beam loss could be observed at the end of a train if no countermeasures were put in place. As the electron cloud is potentially one of the main bottlenecks of the SPS after the upgrade of the LHC injector chain in the framework of the LHC Injectors Upgrade (LIU) project [129], dedicated electron diagnostics devices are installed in the machine to measure the electron flux and spatial electron distribution on surfaces with different coatings, as well as its variation with the time of exposure, i.e. what we call "beaminduced machine scrubbing" [129]. Since 2011, the electron cloud has been also observed routinely in the LHC, causing beam instability and emittance growth at the end of the multi-bunch trains but also additional heat load on the cold beam screens of the arcs as well as of the matching and final focusing quadrupoles [130]. While the effects linked to electron cloud have quickly disappeared for beams with $50 \mathrm{~ns}$ bunch spacing thanks to a relatively rapid beam induced machine scrubbing, running with $25 \mathrm{~ns}$ beams has proved to be rather challenging in this machine. With this type of beams, even after extensive machine scrubbing, the undesired effects of the electron cloud have remained visible on the beam and the machine equipment. In particular, the $25 \mathrm{~ns}$ beam needs to be stabilised with high values of chromaticity in both planes and large octupole settings. Besides, the large heat load on the cold beam screens still remains very close to the capacity of the cryogenic system in nominal operating conditions [131, 132]. While these effects have not prevented running LHC close to the nominal conditions from 2015 to 2018, they could still be a significant showstopper for future operation with double beam current in the High Luminosity LHC era [133]. Concerning lepton machines, the electron cloud is typically associated with a reduction of specific luminosity in e$\mathrm{p}$ colliders and is expected to be one of the main limiting factors for the damping rings of future linear collider projects. The Cornell Electron Storage Ring (CESR) was reconfigured in 2008 as a Test Accelerator (CesrTA) for a program of electron cloud research with lepton beams. With its new local diagnostics for measurement of cloud density and improved instrumentation for the characterization of the beam dynamics of high intensity bunch trains interacting with the cloud, this test facility provided for many years both a benchmark case for the existing simulation codes and testing the effectiveness of several types of countermeasures [134]. Since the processes of secondary electron emission and elastic reflection at the walls play a fundamental role in causing beam induced multipacting, we now shortly describe their key parameters. The true secondary yield for perpendicular incidence, $\delta$, can 
be expressed by a universal function [135]

$$
\delta(x)=\delta_{\max } \frac{s x}{s-1+x^{2}},
$$

where $x=E / E_{\max }$, with $E$ the energy of the incident electron and $E_{\max }$ the energy at which the yield assumes the maximum value, and $s$ is a fit parameter that was measured to be about equal to 1.35 for $\mathrm{LHC} \mathrm{Cu}$ samples [135]. The two variable parameters in Eq. (4.41) are $\delta_{\max }$, the maximum yield, which typically assumes values between about 1.0 and 3.0 for conductive materials (but it can be higher for dielectrics), and $E_{\max }$. For non-normal incidence of the primary electron these two parameters are usually both increased by a factor depending on the cosine of the incidence angle [136]. Elastic reflection of electrons is mostly important at low energies, i.e. below about $20 \mathrm{eV}$. The measured electron reflection probability [122] can be parametrized as

$$
\delta_{\mathrm{el}}(E)=\left(\frac{\sqrt{E}-\sqrt{E+E_{0}}}{\sqrt{E}+\sqrt{E+E_{0}}}\right)^{2},
$$

with only one fit parameter, $E_{0}$. Equation (4.42) implies that the reflection probability approaches one in the limit of vanishing electron energy, even if presently no general consensus has been reached around this point, which is still very controversial, as it is extremely difficult to measure the secondary emission yield at very low energy.

The electron cloud build-up saturates when the electron losses balance the electron generation rate. This can happen either at low bunch charges, when the average neutralization density is reached, or at high bunch currents, when the electrons rapidly accumulate until the kinetic energy of the newly emitted ones becomes too low to let them penetrate into the space charge field of the cloud. Simulations have demonstrated a complex behaviour of the electron cloud equilibrium, which strongly depends on the combination between bunch length, charge, spacing and on the chamber radius. First of all, the saturation phase is generally characterized by an oscillating behaviour of the electron cloud density over the bunch spacing and the amplitude of this oscillation can be very large. Furthermore, in some cases the steady-state value of the electron cloud density has been found not to be monotonically increasing with the bunch intensity. For instance, a beam with $50 \mathrm{~ns}$ spaced bunches in the SPS is predicted to hit its highest electron cloud equilibrium density for bunch populations of about $10^{11} \mathrm{p}$, while this value decreases both for lower and higher intensities.

The electron density decays after the passage of a bunch train (or in the gap between bunch trains) and two different regimes can be distinguished during this phase. In the first one, right after the train passage, the cloud decays quickly due to the space charge effects and the reminiscent energy distribution from the last bunch passage. In the second one, only low energy electrons will be left, 
which move slowly and exhibit a dissipation rate depending on their probability of being elastically reflected at the chamber surface. Due to the second part of the decay evolution, the electron clearing time between bunch trains can become painfully long. Three effects are suspected to be responsible for the long memory and lifetime of the electron cloud. First, nonuniform fields, such as quadrupoles or sextupoles, may act as magnetic bottles and trap electrons for an indefinite time period $[125,137]$. Second, if the probability of elastic reflection really approaches one in the limit of zero electron energy, as is suggested by measurements [122], lowenergetic electrons could survive nearly forever, bouncing back and forth between the chamber walls, independently of the magnetic field. Third, slow ions produced by residual gas ionization have been also suspected to be long lived in the beam chamber and, therefore, possibly cause (or help) electron trapping and long survival time.

\subsubsection{The Electron Cloud Instability}

When a positron/hadron beam interacts electromagnetically with the electron cloud that has formed in the beam chamber, a coherent oscillation of both electrons and beam particles can grow from any small initial perturbation of the beam distribution, e.g. from the statistical fluctuations due to the finite number of beam particles. This instability can be considered as a two-stream instability of the same type as studied in plasma physics. Such instabilities can be very fast, since in the new generation of high intensity rings operating with many closely spaced bunches, the density of electrons can become quickly very large. Even machines operating with bunches spaced by hundreds of ns can actually suffer from electron cloud, because of the long survival time of low energy electrons in the beam pipe. Electron clouds can cause single-bunch instabilities as well as multi-bunch dipole mode instabilities. The multi-bunch instability appears when the electron cloud can carry a sufficiently long memory as to couple subsequent bunches. The single-bunch phenomenon, instead, is driven by a pinched electron cloud, which, over one single passage of the bunch through it, is able to transfer information from an offset bunch head to the bunch tail. Obviously, although this second type of instability is caused by a single-bunch mechanism, it can only occur in multi-bunch operation, since the electron cloud requires a train of several bunches to build up. For single-bunch instabilities caused by multi-bunch built electron clouds, electrons usually only perform a low number of oscillations while the bunch is passing (typically between fractions of unit and few units), and the bunch effectively interacts with a pre-existing cloud produced by the preceding bunches and filling almost uniformly the beam pipe prior to the bunch arrival. The number of electrons does not change appreciably during one bunch passage. In reality, another possible head tail effect resulting into a different type of two-stream instability was observed in some machines operating with long bunches. In this case, the instability is intimately related to an electron cloud from "trailing-edge multipacting", described in the previous section. The electrons 
multiplication happening over the falling edge of the bunch can reach levels as to render the beam unstable. Even coasting beams are not immune from electron cloud problems. The electrons produced from residual gas ionization remain trapped in the transverse potential of the uniform beam and tend to accumulate to very high central density values. The electrons created at the chamber walls (e.g. from beam loss) are accelerated and decelerated in the beam field and eventually hit the chamber with the same energy with which they were emitted. Multipacting can play here a role, since electrons can gain energy and create secondaries when hitting the wall, if the beam line density is perturbed. The interaction of the coasting beam with the electrons can make noise evolve into an unstable coupled oscillation, called e-p instability, which was widely studied already at the beginning of the 70s [138, 139]. While the singlebunch instability described earlier in this section can be treated separately from the build-up of the electron cloud that causes it, in all other cases the two processes are coupled together and need to be solved with a joint model.

Electron cloud instabilities for short bunches have been observed in form of emittance growth and beam loss at the KEKB LER, at the CERN PS, SPS and LHC, and at the PEP-II LER. At the KEKB LER a blow-up of the vertical beam size was already observed at the early commissioning time [140]. This blowup was not accompanied by any coherent beam motion, which could be easily suppressed by transverse feedback and chromaticity, and the blow-up was only seen in multi-bunch operation with a narrow bunch spacing. The single-bunch two-stream instability provided a plausible explanation of the observed beam blow-up [141]. This explanation has since been reinforced by the simultaneous observation of a tune shift along the bunch train, which appears for the same bunches exhibiting vertical size blow-up. Also the experimental evidence that the installation of solenoids around the ring could increase the instability threshold in regular operation shows the relation between electron cloud and the instability. At the CERN SPS the electron cloud has been observed since the ring has been regularly operated with LHC-type bunch trains [142] and it has been held responsible for strong transverse instabilities. In the horizontal plane a low order coupled bunch instability develops within a few tens of turns after injection. In the vertical plane, a single-bunch head tail instability rises on a much shorter time. The reason of the different behavior in the two transverse planes is ascribed to the confinement of the electron cloud mostly in dipole regions, which can limit the intra-bunch electron pinching in the horizontal plane and therefore inhibit the single-bunch mechanism for instability. The horizontal (coupled-bunch) instability is cured by means of a transverse feedback system. Similar to the situation at the KEKB LER, running the SPS at high positive chromaticity can cure the vertical instability [143]. Upstream from the SPS, when the nominal LHC beam was generated by the PS machine, one of the standard signatures of the electron cloud was observed shortly before extraction: a baseline drift in electrostatic devices. However, the beam resided too shortly in the machine to become unstable, even if a dedicated experiment proved the onset of an electron cloud instability on the short bunches, if they are kept in the machine for a sufficiently long time. In the LHC, transverse beam instabilities affecting the last bunches of long $25 \mathrm{~ns}$ trains in both transverse planes have been 
systematically observed at injection [144]. The reason why the instability appears in both planes is that the integrated central density of electrons causing the instability comes mainly from the electron cloud in quadrupole magnets and therefore affects equally both planes. This instability is controlled by means of high chromaticity and high octupole strength. Besides, electron cloud instabilities have been observed also at high energy $(6.5 \mathrm{TeV})$ but mainly in the vertical plane and for values of bunch currents lower than nominal. This has been explained as due to the onset of a central stripe in all the dipoles (appearing when the bunch intensity decays), which leads to an integrated electron density capable of making a $6.5 \mathrm{TeV}$ beam unstable [145]. Concerning long bunches, a great deal of evidence indicates that the primary instability limiting the performance of the LANL-PSR is an e-p instability [146]. Growth of the electron cloud results from multipacting of the electrons on the walls of the vacuum chamber during passage of the trailing edge of the proton beam, when the electrons can receive a net acceleration toward the wall. The instability was controlled by various measures to enhance Landau damping and transverse feedback. In coasting beams, an e-p instability was first observed in the LBNLBevatron [147] and CERN-ISR [139]. While in the Bevatron this instability was combated with active feedback and beam bunching, in the ISR additional pumping was installed to improve the vacuum from 0.1 to 0.01 nTorr and the number of clearing electrodes was increased to sweep away the electrons.

Several analytical approaches have been used to study the electron cloud instability, including few-particles models and an attempt to apply the TMCI theory to the electron cloud wake field, modeled as a broadband resonator. However the most widespread and comprehensive approach makes use of particle tracking simulations with localized electron cloud kicks. The numerical modeling of the interaction between an electron cloud and a particle bunch is discussed in Sect. 4.7. Simulation codes have had the merit to reveal interesting features of the electron cloud instability, which distinguish it from other types of conventional instabilities. For example, the electron cloud wake field is not only a function of the distance between source and probe particles, but it depends on the locations of the two separately. This translates into an impedance with a double frequency dependence [148]. Another interesting finding was that, for constant beam emittances (transverse and longitudinal) and constant bunch length, the electron cloud instability threshold decreases with the beam energy [149]. The reason of this anomalous behaviour is that, although the beam becomes stiffer at higher energies, its transverse sizes become smaller and the pinching effect on the electron cloud is amplified.

Concerning the multi-bunch instability, the usual approach is to calculate the bunch-to-bunch wake field with an electron cloud build-up code (which correctly models the electron cloud dynamics in the space between two bunches) and then apply the multi-bunch analytical formula to assess the threshold for the beam stability. Simulation codes with bunches modeled as single macroparticles, valid for machines operating with short bunches, have been also developed to study numerically the multi-bunch instabilities due to electron cloud in a more selfconsistent manner. 
A number of simulation tools have been developed over the years in order to study the electron cloud single-bunch instability for short bunches via direct particle tracking. The simulations of electron cloud build-up are generally treated separately, since they make use of a weak-strong approach, in which the beam is rigid and is approximated by bunches with static transverse and longitudinal Gaussian distributions, while the electrons are macroparticles. Build-up simulations need to be run prior to the instability simulations, because they provide the necessary input on the transverse distribution of the electron cloud density at saturation just before the arrival of a bunch. A variety of simulation codes are presently available for this purpose [150]. Fully self-consistent computations, in which the cloud generation over a bunch train around the ring as well as the resulting bunch instabilities, are treated by a single program are still under development. The existing simulation programs to study the electron cloud instabilities model the interaction of a single bunch with an electron cloud on successive turns. The cloud is always assumed to be generated by the preceding bunches, and can be considered initially uniform or the distribution is imported from a build-up code. The electrons give rise to a headto-tail wake field, which amplifies any initial small deformation in the bunch offset, e.g. due to the finite number of macroparticles in the simulation. All simulation tools that have been developed for this study are essentially of the strong-strong type, since the purpose is to investigate how the bunch particles are affected by the electron cloud via the continuous interaction. In particular, electrons are always modeled as macroparticles either concentrated at one or several locations along the ring or uniformly smeared along the axis of the machine. The bunch consists of macroparticles or of microbunches with a fixed transverse size. The bunch is then subdivided into slices, which interact in sequence with the electrons of the cloud, creating the distortion of the initially uniform cloud distribution that can affect the body and tail of the bunch. The electric fields of the electrons and of the beam acting mutually on each other are calculated by means of a ParticleIn-Cell (PIC) algorithm. The transformation of the 6D phase-space vectors of the beam particles between two kick points is achieved using the appropriate transport matrices or nonlinear tracking. The field of the electron cloud acting on itself can be optionally included, but in general does not seem to play a significant role in this type of mechanisms and hence it is neglected. For the purpose of studying the interplay of electron cloud instability with other mechanisms, the simulation codes contain synchrotron motion, chromaticity and usually additional options to model the action of an independent impedance source beside the electron cloud, as well as space charge and detuning with amplitude.

\subsubsection{Mitigation and Suppression}

There are at least three possible actions to reduce, or even suppress, the electron cloud: (i) reducing the production rate of primary electrons or confining their motion to a region where they are not likely to do any harm; (ii) eliminating the possibility 
of multipacting by lowering the SEY via surface treatment; (iii) alleviating the effect on the beam or on the diagnostics. In most cases a combined approach is desirable, that's why most machines affected by electron cloud problems have usually chosen to implement more than one of these mitigating techniques. The primary production of photoelectrons needs to be reduced, because it may be so high that the electron cloud could reach saturation within a few bunch passages even without any multipacting. This is the case at KEKB, the photon factory, if no countermeasures were taken. The obvious solution is an antechamber to intercept most of the synchrotron radiation, or also photon absorbers (as those designed for the CLIC Damping Rings). For dipole fields, a saw-tooth pattern impressed on the chamber wall, as was implemented for the LHC (actually on the beam screen that forms the inner part of the chamber and serves to protect the cold bore of the magnets from synchrotron radiation), is used for effectively reducing the photon reflectivity thanks to the perpendicular impact. Weak solenoids of the order of 50 $\mathrm{G}$ are a possibility in field-free regions, which was successfully implemented in the straight sections of KEKB and in RHIC. The solenoids do not really affect the photoemission process, but they keep the photoelectrons close to the wall and, thus, strongly mitigate the subsequent beam-electron interaction. Since the gas ionization rate is linearly proportional to the vacuum pressure in the beam chamber, the number of electrons created by gas ionization can only be reduced by significant factors improving the vacuum. If field ionization is important, however, a possible cure would be lengthening the bunches, though this will mainly be a concern for future projects such as linear colliders or X-ray FELs operating with positrons. Electrons generated by beam loss can be controlled if the localization of the losses is known with good precision. For example, electrons produced by the beam losses at a collimator can be controlled by solenoids or clearing electrodes. A large number of electrons is also generated at the injection stripping foils, for accelerators employing charge-exchange injection. At the SNS, a 10-kV clearing voltage is applied to channel the electrons liberated at the stripping foil onto a collector plate that is monitored by a TV camera, while solenoids are used along the collimator straights.

The reduction of the SEY of the inner wall of the beam chamber can be achieved in different manners. First of all, a serendipitous feature of the electron cloud build up in an accelerator's chamber is that, while the electrons hit the beam chamber with high energy and multiply, they also 'scrub' the surface by first removing layers of impurities responsible for high SEY values and eventually graphitising the surface with a further reduction of the SEY from that of the pure metal [151]. This means that, if a method is found to run an accelerator with electron cloud and stable beam, e.g. by stabilising the beam against the electron cloud through appropriate machine settings, it will be the electron cloud itself to gradually lower the SEY of the inner wall of the chamber and eventually turn itself off. This obviously relies on that the final SEY reachable through scrubbing is below the value that sets off the electron cloud build up in the operational configuration. Besides, it may take a significant amount of time to reach this condition, because the electron flux is decreasing while scrubbing and the electron doses required to perform further SEY reduction steps are 
also exponentially increasing when moving to SEYs below 1.3-1.4. The technique described here is what we call 'beam induced machine scrubbing' and machine like the SPS and LHC fully rely on it to run successfully with $25 \mathrm{~ns}$ spaced beams. While beam induced scrubbing is an important option for already built machines, coatings with intrinsically low SEY materials can be envisaged at the design stage to limit the creation of an electron cloud in future machines. A well established method to reduce multipacting is coating with $\mathrm{TiN}$, a material whose secondary emission yield becomes quickly low after some conditioning (through illumination under synchrotron light). The thickness of the coating must be of the order of a $\mu \mathrm{m}$, such as not to alter the resistive impedance seen by the beam. A more favorable getter material made from TiZrV, called Non-Evaporable Getter (NEG), was developed at CERN. This getter material is characterized by its greater structural stability than TiN, its pumping capability and its low activation temperature. The warm sections of the LHC, about $10 \%$ of the circumference, have been coated with NEG. The NEG coating was also tested at several light source insertion devices, where circumstantial evidence suggests an increase in the effective impedance, presumably due to a larger surface roughness and low conductivity. The additional contributions to the ring impedance from the surface roughness and low conductivity impedance of the coating layer is of no concern for the longer proton bunches in the LHC, but could significantly affect the stability of the short positron beams in the Damping Rings of a future linear collider. From 2007 on, new efforts have been put on the search for coating materials that do not require high temperature activation and do not suffer from aging. In particular, amorphous carbon (a-C) thin films, deposited with d.c. magnetron sputtering, have shown to possess all these qualities. Besides, their maximum secondary emission yields, measured in the lab, reach values even below one and the films are also stable against mechanical stress. Testing of a-C coating in accelerator environments (SPS and Cesr-TA) has demonstrated all these features. Another method to reduce the secondary emission yield of a surface is to use a naturally rough material. Here the SEY reduction is a geometrical effect due to the high probability of quick re-absorption of the electrons emitted with low energy.

Multipacting can also be suppressed by solenoids, though one should pay attention to the possibility of exciting undesired cyclotron resonances. Electric clearing fields are an efficient cure, as shown both in simulations and measurements of electron cloud in the CERN PS. They were already used to cure electron-proton instabilities for the coasting proton beams in the CERN ISR during the early 70s. At the SNS operating with long proton bunches all BPMs can be biased with a clearing voltage of $1 \mathrm{kV}$. To be effective for the multipacting experienced by short bunches with close spacing, the clearing electrodes must be mounted all around the ring, in distances of a few tens of $\mathrm{cm}$ and voltages of the order $1 \mathrm{kV}$ are probably required. The impedance introduced by many such devices could be prohibitive, as it appeared to be the case in the DA $\Phi$ NE positron ring with the very first clearing electrode design. Other options for a practical implementation of electric clearing fields may be splitting the beam pipe into a top and bottom half, isolated from each other and held at different potential. Biasing the two jaws of a collimator 
against each other is a similar idea. Recently, there is a growing interest towards the suppression of multipacting by means of grooves on the chamber wall. This technique, first tested in simulations, has proven to be efficient in KEKB and CesrTA. Similarly to a rough surface, but in a controlled way and on a macroscopic scale, these grooves essentially act as electron traps. Angle and depth of the grooves are key parameters and specifications are different in dipole or field-free regions. Proper tailoring of the bunch filling patterns (bunch spacing, bunch trains and bunch charges) is yet another way of achieving an acceptable electron density. Examples include the actual bunch spacing chosen for PEP-II and KEKB operation, which are twice or three times the design spacing, and satellite bunches proposed for the LHC [152]. Gaps within or between trains can lower the density and reset the cloud at least to some extent. Extensive studies of the electron cloud formation as a function of the bunch filling patterns were also carried out at RHIC, in which the optimization could be found using the maps approach to quickly scan the build-up for different configurations.

The electron cloud causes a large variety of undesired effects. Common stabilising measures can be taken against the resulting instabilities, which include transverse bunch-to-bunch feedback, increased chromaticity, Landau-damping octupoles, intra-bunch head-tail feedback, and linear coupling. All these measures are anyway necessary when a machine is operating in beam-induced scrubbing mode. Degradation of diagnostics signals due to impacting electrons can be also overcome with local solenoid windings.

\subsection{Beam-Beam Effects}

W. Herr

\subsubsection{Introduction}

The problem of the beam-beam interaction is the subject of many studies since the introduction of the first particle colliders [153]. It has been and will be one of the most important limits to the performance and therefore attracts the interest at the design stage of a new colliding beams facility. A particle beam is a collection of a large number of charges and represents an electromagnetic potential for other charges. It will therefore exert forces on itself and other beams. The forces are most important for high density beams, i.e. high intensity and small beam sizes, which are the key to high luminosity.

The electromagnetic forces from particle beams are very non-linear and result in a wide spectrum of consequences for the beam dynamics. Furthermore, as a result of the interaction, the charge distribution creating the disturbing fields can change as well. This has to be taken into account in the evaluation of beam- 
beam effects and in general a self-consistent treatment is required. Although we now have a good qualitative understanding of the various phenomena, a complete theory does not exist and exact predictions are still difficult. Numerical techniques such as computer simulations have been used with great success to improve the picture on some aspects of the beam-beam interaction while for other problems the available models are not fully satisfactory in their predictive power [154].

\subsubsection{Beam-Beam Force}

In the rest frame of a beam we have only electrostatic fields and to find the forces on other moving charges, we have to transform the fields into the moving frame and to calculate the Lorentz forces (see [153, 155-160] and references therein).

The fields are obtained by integrating over the charge distributions. The forces can be defocusing or focusing since the test particle can have the same or opposite charge with respect to the beam producing the forces.

The distribution of particles producing the fields can follow various functions, leading to different fields and forces. It is not always possible to integrate the distribution to arrive at an analytical expression for the forces in which case either an approximation or numerical methods have to be used. This is in particular true for hadron beams, which usually do not experience significant synchrotron radiation and damping. For $\mathrm{e}^{-} \mathrm{e}^{+}$colliders the distribution functions are most likely Gaussian with truncated tails.

In the two-dimensional case of a beam with bi-Gaussian beam density distributions in the transverse planes, i.e. $\rho(x, y)=\rho_{x}(x) \rho_{y}(y)$ with r.m.s. of $\sigma_{x}$ and $\sigma_{y}$

$$
\rho_{u}(u)=\frac{1}{\sigma_{u} \sqrt{2 \pi}} \exp \left(-\frac{u^{2}}{2 \sigma_{u}^{2}}\right) \text { where } u=x, y
$$

one can give the two-dimensional potential $U\left(x, y, \sigma_{x}, \sigma_{y}\right)$ as a closed expression

$$
U\left(x, y, \sigma_{x}, \sigma_{y}\right)=\frac{n e}{4 \pi \varepsilon_{0}} \int_{0}^{\infty} \frac{\exp \left(-\frac{x^{2}}{2 \sigma_{x}^{2}+q}-\frac{y^{2}}{2 \sigma_{y}^{2}+q}\right)}{\sqrt{\left(2 \sigma_{x}^{2}+q\right)\left(2 \sigma_{y}^{2}+q\right)}} d q
$$

where $n$ is the line density of particles in the beam, $e$ is the elementary charge and $\varepsilon_{0}$ the permittivity of free space [159]. From the potential one can derive the transverse fields $\vec{E}$ by taking the gradient $\vec{E}=-\nabla U\left(x, y, \sigma_{x}, \sigma_{y}\right)$. 


\subsubsection{Elliptical Beams}

For the above case of bi-Gaussian distributions (i.e. elliptical beams with $\sigma_{x} \neq \sigma_{y}$ ) the fields can be derived and for the case of $\sigma_{x}>\sigma_{y}$ we have [160]

$$
\begin{aligned}
E_{x}= & \frac{n e}{2 \varepsilon_{0} \sqrt{2 \pi\left(\sigma_{x}^{2}-\sigma_{y}^{2}\right)}} \operatorname{Im} \\
& \left.\times\left[\operatorname{erf} \frac{x+i y}{\sqrt{2\left(\sigma_{x}^{2}-\sigma_{y}^{2}\right)}}\right)-\exp \left(-\frac{x^{2}}{2 \sigma_{x}^{2}}+\frac{y^{2}}{2 \sigma_{y}^{2}}\right) \operatorname{erf}\left(\frac{x \frac{\sigma_{y}}{\sigma_{x}}+i y \frac{\sigma_{x}}{\sigma_{y}}}{\sqrt{2\left(\sigma_{x}^{2}-\sigma_{y}^{2}\right)}}\right)\right] \\
E_{y}= & \left.\left.\frac{n e}{2 \varepsilon_{0} \sqrt{2 \pi\left(\sigma_{x}^{2}-\sigma_{y}^{2}\right)} \operatorname{Re}}\right)-\exp \left(-\frac{x^{2}}{2 \sigma_{x}^{2}}+\frac{y^{2}}{2 \sigma_{y}^{2}}\right) \operatorname{erf}\left(\frac{x \frac{\sigma_{y}}{\sigma_{x}}+i y \frac{\sigma_{x}}{\sigma_{y}}}{\sqrt{2\left(\sigma_{x}^{2}-\sigma_{y}^{2}\right)}}\right)\right] \\
& \times\left[\operatorname{erf}\left(\frac{x+i y}{2\left(\sigma_{x}^{2}-\sigma_{y}^{2}\right)}\right)\right]
\end{aligned}
$$

The function $\operatorname{erf}(t)$ is the complex error function

$$
\operatorname{erf}(t)=\exp \left(-t^{2}\right)\left[1+\frac{2 i}{\sqrt{\pi}} \int_{0}^{t} \exp \left(z^{2}\right) d z\right]
$$

The magnetic field components follow from

$$
B_{y}=-\beta \frac{E_{x}}{c} \text { and } B_{x}=\beta \frac{E_{y}}{c}
$$

The Lorentz force acting on a particle with charge $q$ is finally

$$
\vec{F}=q(\vec{E}+\vec{v} \times \vec{B})
$$

\subsubsection{Round Beams}

With the simplifying assumption of round beams $\left(\sigma_{x}=\sigma_{y}=\sigma\right)$, one can re-write (4.49) in cylindrical coordinates

$$
\vec{F}=q\left(E_{r}+\beta c B_{\phi}\right) \times \vec{r}
$$


From (4.44) and with $r^{2}=x^{2}+y^{2}$ one can immediately write the fields from (4.50) as

$$
E_{r}=-\frac{n e}{4 \pi \varepsilon_{0}} \frac{\delta}{\delta_{r}} \int_{0}^{\infty} \frac{\exp \left(-\frac{r^{2}}{2 \sigma^{2}+q}\right)}{2 \sigma^{2}+q} d q
$$

and

$$
B_{\phi}=-\frac{n e \beta c \mu_{0}}{4 \pi} \frac{\delta}{\delta_{r}} \int_{0}^{\infty} \frac{\exp \left(-\frac{r^{2}}{2 \sigma^{2}+q}\right)}{2 \sigma^{2}+q} d q
$$

$\delta$ stands for derivative (Eqs. 4.51 and 4.52) and $\mu_{0}$ is the permeability of free space (Eq. 4.52).

We find from (4.51) and (4.52) that the force (4.50) has only a radial component. The expressions (4.51) and (4.52) can easily be evaluated when the derivative is done first and $1 /\left(2 \sigma^{2}+q\right)$ is used as integration variable. We can now express the radial force in a closed form (using $\varepsilon_{0} \mu_{0}=c^{-2}$ )

$$
F_{r}(r)=-\frac{n e^{2}\left(1+\beta^{2}\right)}{2 \pi \varepsilon_{0}} \frac{1}{r}\left[1-\exp \left(-\frac{r^{2}}{2 \sigma^{2}}\right)\right]
$$

and for the Cartesian components in the two transverse planes we get

$$
F_{x}(r)=-\frac{n e^{2}\left(1+\beta^{2}\right)}{2 \pi \varepsilon_{0}} \frac{x}{r^{2}}\left[1-\exp \left(-\frac{r^{2}}{2 \sigma^{2}}\right)\right]
$$

and

$$
F_{y}(r)=-\frac{n e^{2}\left(1+\beta^{2}\right)}{2 \pi \varepsilon_{0}} \frac{y}{r^{2}}\left[1-\exp \left(-\frac{r^{2}}{2 \sigma^{2}}\right)\right]
$$

The forces (4.54) and (4.55) are computed when the charges of the test particle and the opposing beam have opposite signs. For equally charged beams the forces change signs. For small amplitudes the force is approximately linear and a particle crossing a beam at small amplitudes will experience a linear field. This results in a change of the tune like in a quadrupole. At larger amplitudes (i.e. above $\sim 1 \sigma$ ) the force deviates strongly from this linear behaviour. Particles at larger amplitudes will also experience a tune change, however this tune change will depend on the amplitude. Already from the analytical form (4.55) one can see that the beam-beam force includes higher multipoles. 


\subsubsection{Incoherent Effects: Single Particle Effects}

The force we have derived is the force of a beam on a single test particle. It can be used to study single particle or incoherent effects. For that we treat a particle crossing a beam like it was moving through a static electromagnetic lens. We have to expect all effects that are known from resonance and non-linear theory such as

- Unstable and/or irregular motion

- Beam blow up or bad lifetime

\subsubsection{Beam-Beam Parameter}

We can derive the linear tune shift of a small amplitude particle crossing a round beam of a finite length. We use the force to calculate the kick it receives from the opposing beam, i.e. the change of the slope of the particle trajectory. Starting from the two-dimensional force and multiplying with the longitudinal distribution which depends on both position $s$ and time $t$, and assuming a Gaussian shape with a width of $\sigma_{s}$

$$
F_{r}(r, s, t)=-\frac{N e^{2}\left(1+\beta^{2}\right)}{\sqrt{(2 \pi)^{3}} \varepsilon_{0} \sigma_{s}} \frac{1}{r}\left[1-\exp \left(-\frac{r^{2}}{2 \sigma^{2}}\right)\right] \exp \left[-\frac{(s+v t)^{2}}{2 \sigma_{s}^{2}}\right]
$$

Now $N$ is the total number of particles. We make use of Newton's law and integrate over the collision to get the radial deflection

$$
\Delta r^{\prime}=\frac{1}{m c \beta \gamma} \int_{-\infty}^{\infty} F_{r}(r, s, t) d t
$$

The radial kick $\Delta r^{\prime}$ a particle with a radial distance $r$ from the opposing beam centre receives is then

$$
\Delta r^{\prime}=-\frac{2 N r_{0}}{\gamma} \frac{1}{r}\left[1-\exp \left(-\frac{r^{2}}{2 \sigma^{2}}\right)\right]
$$

where I have re-written the constants and use the classical particle radius

$$
r_{0}=\frac{e^{2}}{4 \pi \varepsilon_{0} m c^{2}}
$$

where $m$ is the mass of the particle. After the integration along the bunch length, $N$ is the total number of particles. For small amplitudes $r$ one can derive the asymptotic 
limit

$$
\left.\Delta r^{\prime}\right|_{r \rightarrow 0}=-\frac{N r_{0} r}{\gamma \sigma^{2}}=-r f
$$

This limit is the slope of the force at $r=0$ and the force becomes linear with a focal length as the proportionality factor.

It is well known how the focal length relates to a tune change and one can derive a quantity $\xi$ which is known as the linear beam-beam parameter

$$
\xi=\frac{N r_{0} \beta^{*}}{4 \pi \gamma \sigma^{2}}
$$

$r_{0}$ is the classical particle radius, (e.g.: $r_{e}, r_{p}$ ) and $\beta^{*}$ is the optical amplitude function ( $\beta$-function) at the interaction point.

For small values of $\xi$ and a tune far enough away from linear resonances this parameter is equal to the linear tune shift $\Delta Q$.

The beam-beam parameter can be generalized for the case of non-round beams and becomes

$$
\xi_{x, y}=\frac{N r_{0} \beta_{x, y}^{*}}{2 \pi \gamma \sigma_{x, y}\left(\sigma_{x}+\sigma_{y}\right)}
$$

The beam-beam parameter is often used to quantify the strength of the beambeam interaction, however it does not reflect the non-linear nature.

\subsubsection{Non-linear Effects}

Since the beam-beam forces are strongly non-linear, the study of beam-beam effects encompasses the entire field of non-linear dynamics (see earlier chapter) as well as collective effects. First, we briefly discuss the immediate effect of the nonlinearity of the beam-beam force on a single particle. It manifests as an amplitude dependent tune shift and for a beam with many particles as a tune spread. The instantaneous tune shift of a particle when it crosses the other beam is related to the derivative of the force with respect to the amplitude $\delta F / \delta x$. For a particle performing an oscillation with a given amplitude the tune shift is calculated by averaging the slopes of the force over the range (i.e. the phases) of the particle's oscillation amplitudes. An elegant calculation can be done using the Hamiltonian formalism [156] developed for non-linear dynamics and as demonstrated in the chapter on non-linear dynamics using the Lie formalism. We get the formula for the non-linear detuning with the amplitude $J$

$$
\Delta Q(J)=\xi \frac{2}{J}\left[1-I_{0}\left(\frac{J}{2}\right) \mathrm{e}^{-\frac{J}{2}}\right]
$$



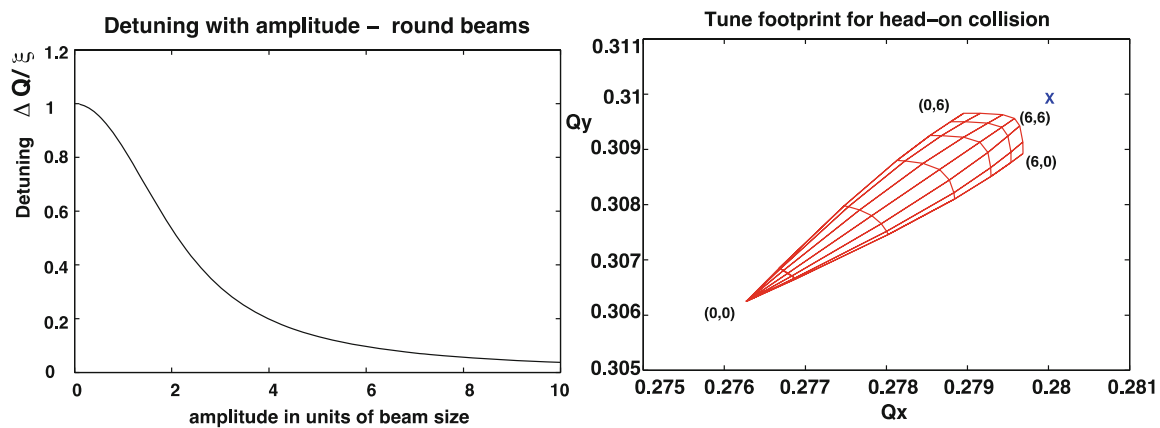

Fig. 4.22 Tune shift (non-linear detuning) as a function of the amplitude (left) and 2-dimensional tune footprint (right)

where $I_{0}(x)$ is the modified Bessel function and $J=\varepsilon \beta / 2 \sigma^{2}$ in the usual units. Here $\varepsilon$ is the particle "emittance" and not the beam emittance.

In the 2-dimensional case, the tune shifts $\left(\Delta Q_{x}, \Delta Q_{y}\right)$ of a particle with amplitudes $x$ and $y$ depend on both, horizontal and vertical amplitudes. The detuning must be computed and presented in a 2-dimensional form, i.e. the amplitude $(x, y)$ is mapped into the tune space $\left(Q_{x}, Q_{y}\right)$ or alternatively to the 2-dimensional tune change $\left(\Delta Q_{x}, \Delta Q_{y}\right)$. Such a presentation is usually called a "tune footprint" and an example is shown in Fig. 4.22(right) and it maps the amplitudes into the tune space and each "knot" of the mesh corresponds to a pair of amplitudes. Amplitudes between 0 and $6 \sigma$ in both planes are used. The cross indicates the original, unperturbed tunes without the beam-beam interaction.

The maximum tune spread for a single head-on collision is equal to the tune shift of a particle with small amplitudes and for small tune shifts equal to the beambeam parameter $\xi$. In the simple case of a single head-on collision the parameter $\xi$ is therefore a measure for the tune spread in the beam.

\subsubsection{Beam Stability}

When the beam-beam interaction becomes too strong, the beam can become unstable or the beam dynamics is strongly distorted. One can distinguish different types of distortions and a few examples are

- Non-linear motion can become stochastic and can result in a reduction of the dynamic aperture and particle loss and bad lifetime.

- Distortion of beam optics: dynamic beta (LEP) [161].

- Vertical blow-up above the so-called beam-beam limit.

Since the beam-beam force is very non-linear, the motion can become "chaotic". This often leads to a reduction of the available dynamic aperture. The dynamic aperture is the maximum amplitude where the beam remains stable. Particles outside 
the dynamic aperture are eventually lost. The dynamic aperture is usually evaluated by tracking particles with a computer program through the machine where they experience the fields from the machine elements and other effects such as wake fields or the beam-beam interaction.

Since the beam-beam interaction is basically a very non-linear lens in the machine, it distorts the optical properties and it may create a noticeable beating of the $\beta$-function around the whole machine and at the location of the beambeam interaction itself. This can be approximated by inserting a quadrupole which produces the same tune shift at the position of the beam-beam interaction. The

r.m.s. beam size at the collision point is now proportional to $\sqrt{\beta_{p}^{*}}$, where $\beta_{p}^{*}$ is the perturbed $\beta$-function which can be significantly different from the unperturbed $\beta$ function $\beta^{*}$. This in turn changes the strength of the beam-beam interaction and the parameters have to be found in a self-consistent form. This is called the dynamic beta effect. This is a first deviation from our assumption that the beams are static non-linear lenses. A strong dynamic beta effect was found in LEP [161] due to its very large tune shift parameters.

Another effect that can be observed in particular in $\mathrm{e}^{+} \mathrm{e}^{-}$colliders is the blow up of the emittance which naturally limits the reachable beam-beam tune shifts.

\subsubsection{Beam-Beam Limit}

In $\mathrm{e}^{+} \mathrm{e}^{-}$colliders the beam sizes are usually an equilibrium between the damping due to the synchrotron radiation and heating mechanisms such as quantum excitation, intra-beam scattering and very importantly, the beam-beam effect. This leads to a behaviour that is not observed in a hadron collider. When the luminosity is plotted as a function of the beam intensity, it should increase approximately as the current squared [162], in agreement with

$$
\mathcal{L}=\frac{N^{2} k f}{4 \pi \sigma_{x} \sigma_{y}}
$$

Here $k$ is the number of bunches per beam and $f$ the revolution frequency [162]. At the same time the beam-beam parameter $\xi$ should increase linearly with the beam intensity according to (4.60)

$$
\xi_{y}=\frac{N r_{e} \beta_{y}}{2 \pi \gamma \sigma_{y}\left(\sigma_{x}+\sigma_{y}\right)}
$$

In all $\mathrm{e}^{+} \mathrm{e}^{-}$colliders the observation can be made that above a certain current, the luminosity increases approximately proportional to the current, or at least much less than with the second power. Another observation is that at the same value of the intensity the beam-beam parameter $\xi$ saturates. This limiting value of $\xi$ is commonly known as the beam-beam limit. 
When we re-write the luminosity as

$$
\mathcal{L}=\frac{N^{2} k f}{4 \pi \sigma_{x} \sigma_{y}}=\frac{N k f}{4 \pi \sigma_{x}} \frac{N}{\sigma_{y}}
$$

we get an idea of what is happening. In $\mathrm{e}^{+} \mathrm{e}^{-}$colliders the horizontal beam size $\sigma_{x}$ is usually much larger than the vertical beam size $\sigma_{y}$ and changes very little. In order for the luminosity to increase proportionally to the intensity $N$, the factor $N / \sigma_{y}$ must be constant. This implies that with increasing current the vertical beam size increases in proportion above the beam-beam limit. This has been observed in all $\mathrm{e}^{+} \mathrm{e}^{-}$colliders and since the vertical beam size is usually small, this emittance growth can be very substantial before the life time of the beam is affected or beam losses are observed [163].

The dynamics of machines with high synchrotron radiation is dominated by the damping properties and the beam-beam limit is not a universal constant nor can it be predicted. Simulation of beams with many particles can provide an idea of the order of magnitude [164, 165].

\subsubsection{Studies of Head-on Collisions at the LHC}

The layout of experimental regions in the LHC is shown in Fig. 4.23. The beams travel in separate vacuum chambers and cross in the experimental areas where they share a common beam pipe. In these common regions the beams experience headon collisions as well as a large number of long range beam-beam encounters [166]. This arrangement together with the bunch filling scheme of the LHC as shown in Fig. $4.24[166,167]$ leads to very different collision pattern for different bunches, often referred to as "PACMAN" bunches. The number of both, head-on as well as long range encounters, can be very different for different bunches in the bunch trains and lead to a different integrated beam-beam effect [167]. This was always a worry in the LHC design and the effects have been observed in an early stage of the commissioning. Strategies have been provided to minimize these effects, e.g. different planes for the crossing angles $[166,167]$.

\subsubsection{PACMAN Bunches}

The bunches in the LHC do not form a continuous train of equidistant bunches spaced by $25 \mathrm{~ns}$, but some empty space must be provided to allow for the rise time of kickers (Fig. 4.24). These gaps and the number of bunches per train are determined by requirements from the LHC injectors. The whole LHC bunch pattern is composed of 39 smaller trains (each with 72 bunches) separated by gaps of various length followed by a large abort gap for the dump kicker. Due to the symmetry, 


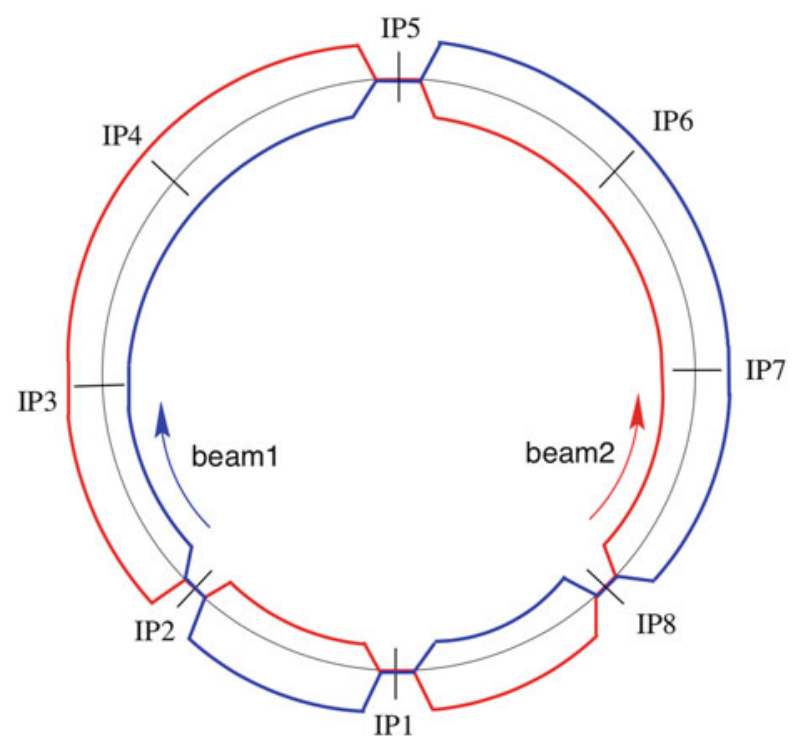

Fig. 4.23 Layout of the experimental collision points in the LHC [166]

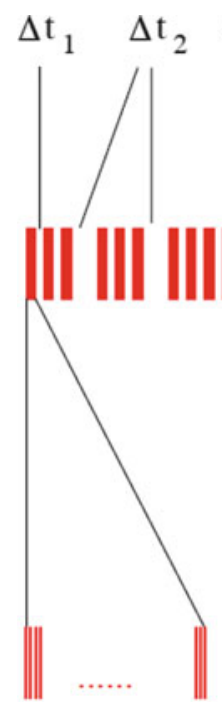

72 bunches

$$
\begin{aligned}
& \Delta t_{1} \quad 8 \text { bunches missing } \\
& \Delta t_{2} \quad 38 \text { bunches missing } \\
& \Delta t_{3} \quad 39 \text { bunches missing } \\
& \Delta t_{4} \quad 119 \text { bunches missing }
\end{aligned}
$$

total number of bunches: 2808

Fig. 4.24 Bunch filling scheme of the nominal LHC

bunches normally meet other bunches at the head-on collision point. For the longrange interactions this is no longer the case. Bunches at the beginning and at the end of a small train will encounter a hole and as a result experience fewer long-range 
interactions than bunches from the middle of a train [168]. Bunches with fewer longrange interactions have a very different integrated beam-beam effect and a different dynamics must be expected. In particular they will have a different tune and occupy a different area in the working diagram, therefore may be susceptible to resonances which can be avoided for nominal bunches. The overall space needed in the working diagram is therefore largely increased [166, 168].

\subsubsection{Head-on Beam-Beam Tune Shift}

The nominal LHC parameters have been chosen to reach the design luminosity of $10^{34} \mathrm{~cm}^{-2} \mathrm{~s}^{-1}$ [166]. The main parameters relevant for beam-beam effects are summarized in Table 4.1. At a very early stage of the LHC operation it was tested whether the nominal beam-beam parameters can be achieved. After this has been successfully demonstrated, we have performed a dedicated experiment to test the achievable beam-beam tune shift. To that purpose we have filled the LHC with single bunches per beam, colliding in IP1 and IP5 (see Fig. 4.23). We have used bunch intensities of $\sim 1.9 \times 10^{11} \mathrm{p} / \mathrm{b}$, i.e. well above the nominal and the emittances have been reduced below $1.20 \mu \mathrm{m}$ in both planes. It was shown that such bunches can be collided in both interaction points without significant losses or emittance increase [169] and we have demonstrated that a beam-beam tune shift of 0.017 for a single interaction and an integrated tune shift of 0.034 for both collision was possible. These tune shifts have been obtained in the absence of any long range encounters and it should be expected that the operationally possible tune shifts are lower.

\subsubsection{Effect of Number of Head-on Collisions}

Due to the filling pattern in the LHC, different bunches experience different numbers of head-on as well as long range interactions. Details are given in another contribution [170]. In Fig. 4.25 we show as illustration the losses of bunches with very different $(0-3)$ numbers of head-on collisions. The data was taken during a

Table 4.1 LHC nominal parameters and achieved during operation and experiments in $2010 / 2011$

\begin{tabular}{l|l|l}
\hline Parameter & Nominal & Achieved \\
\hline Intensity (p/bunch) & $1.15 \times 10^{11}$ & $2.3 \times 10^{11}$ \\
\hline Emittance & $3.75 \mu \mathrm{m}$ & $\leq 2.00 \mu \mathrm{m}$ \\
\hline$\beta_{*}$ & $0.55 \mathrm{~m}$ & $1.5 \mathrm{~m}$ \\
\hline$\xi / \mathrm{IP}$ & 0.0035 & 0.0170 \\
\hline Bunch spacing & $25 \mathrm{~ns}$ & $50 \mathrm{~ns}$ \\
\hline Bunches/beam & 2808 & 1380 \\
\hline
\end{tabular}



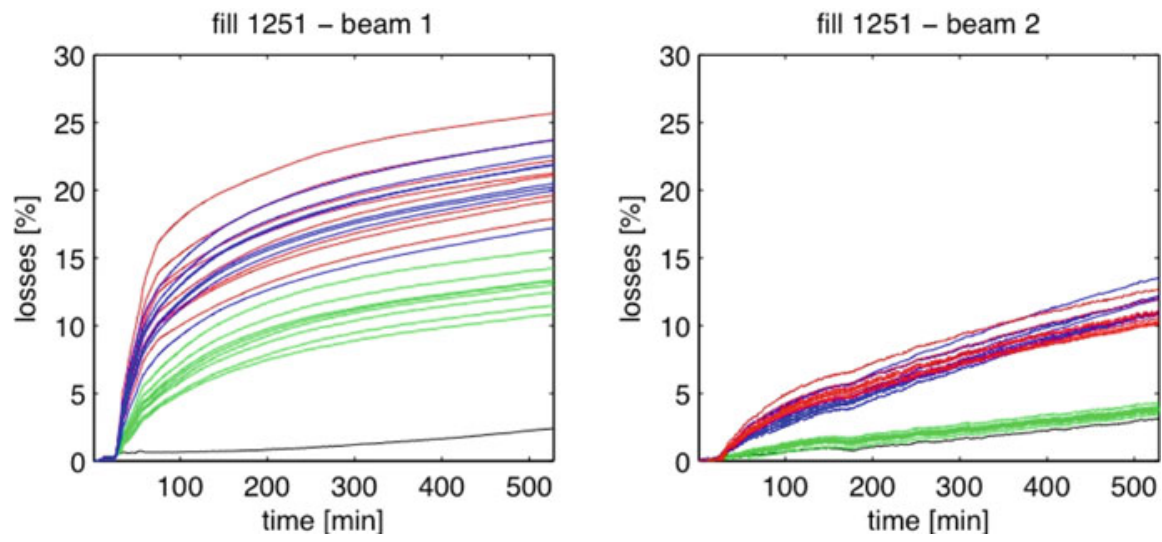

Fig. 4.25 Losses of bunches with different number of head-on collisions [170]. Numerology: blue ( 3 collisions), red ( 2 collisions), green ( 1 collision), black (no collision)

regular operational fill of $10 \mathrm{~h}$ duration. The correlation between losses and number of head-on collisions is apparent and a more detailed analysis is found in [170]. The transverse emittances during normal operation are larger $(\sim 2.5 \mu \mathrm{m})$ than in the head-on test. In a second experiment we increased the bunch intensity further to $\sim 2.3 \times 10^{11} \mathrm{p} / \mathrm{b}$ with emittances of $\sim 1.80 \mu \mathrm{m}$. Although the tune shift was slightly lower than in the previous experiment (0.015), the lifetime was worse. We interprete these results as losses of particles at large amplitudes. This is supported by the observation that the strongest losses occur at the very beginning of a fill (Fig. 4.25).

\subsubsection{Crossing Angle and Long Range Interactions}

To reach the highest luminosity, it is desirable to operate a collider with as many bunches as possible since the luminosity is proportional to their number (4.62) [162].

In a single ring collider such as the SPS, Tevatron or LEP, the operation with $k$ bunches leads to $2 k$ collision points. When $k$ is a large number, most of them are unwanted and must be avoided to reduce the perturbation due to the beambeam effects. Various schemes have been used to avoid these unwanted "parasitic" interactions. In the SPS, Tevatron and in LEP so-called Pretzel schemes were used. When the bunches are equidistant, this is the most promising method. When two beams of opposite charge travel in the same beam pipe, they can be moved onto separate orbits using electrostatic separators. In a well-defined configuration the two beams cross when the beams are separated. To avoid a separation around the whole machine, the bunches can be arranged in so-called trains of bunches following each other closely. In that case a separation with electrostatic separators is only needed 
around the interaction regions. Such a scheme was used in LEP in the second phase [171].

Contrary to the majority of the colliders, the LHC collides particles of the same type which therefore must travel in separate beam pipes. At the collision points of the LHC the two beams are brought together and into collision. During that process it is unavoidable that the beams travel in a common vacuum chamber for more than $120 \mathrm{~m}$. In the LHC the distance between the bunches is only $25 \mathrm{~ns}$ and therefore the bunches will meet in this region. In order to avoid the collisions, the bunches collide at a small crossing angle of $285 \mu \mathrm{rad}$. While two bunches collide at a small angle (quasi head-on) at the centre, the other bunches are kept separated by the crossing angle. However, since they travel in a common beam pipe, the bunches still feel the electromagnetic forces from the bunches of the opposite beam. When the separation is large enough, these so-called long-range interactions should be weak.

\subsubsection{Long-Range Beam-Beam Effects}

Although the long-range interactions distort the beams much less than a headon interaction, their large number and some particular properties require careful studies:

- They break the symmetry between planes.

- While the effect of head-on collisions is strongest for small amplitude particles, they mostly affect particles at large amplitudes.

- The tune shift caused by long-range interactions has opposite sign in the plane of separation compared to the head-on tune shift.

- They cause changes of the closed orbit [153].

- They largely enhance so-called PACMAN effects [168].

\subsubsection{Opposite Sign Tune Shift}

The opposite sign of the tune shift can easily be understood when we average the oscillation of a small amplitude particle as it samples the focusing force of the beam-beam interaction. When the separation is larger than $\sim 1.5 \sigma$, the focusing (slope of the force as a function of the amplitude) changes the sign and the resulting tune shift assumes the opposite sign.

To some extend this property could be used to partially compensate long-range interactions when a configuration is used where the beams are separated in the horizontal plane in one interaction region and in the vertical plane in another one. 


\subsubsection{Strength of Long-Range Interactions}

Assuming a separation $d$ in the horizontal plane, the kicks in the two planes can be written as

$$
\Delta x^{\prime}=-\frac{2 N r_{0}}{\gamma} \frac{(x+d)}{r^{2}}\left(1-\mathrm{e}^{-\frac{r^{2}}{2 \sigma^{2}}}\right)
$$

with $r^{2}=(x+d)^{2}+y^{2}$. The equivalent formula for the plane orthogonal to the separation is

$$
\Delta y^{\prime}=-\frac{2 N r_{0}}{\gamma} \frac{y}{r^{2}}\left(1-\mathrm{e}^{-\frac{r^{2}}{2 \sigma^{2}}}\right)
$$

The effect of long-range interactions must strongly depend on the separation. The calculation shows that the tune spread $\Delta Q_{l r}$ from long-range interactions alone follows an approximate scaling (for large enough separation, i.e. above $\sim 6 \sigma$ )

$$
\Delta Q_{l r} \propto-\frac{N}{d^{2}}
$$

where $N$ is the bunch intensity and $d$ the separation. Small changes in the separation can therefore result in significant differences. Since the symmetry between the two planes is broken, the resulting footprint shows no symmetry. In fact, the tune shifts have different signs for $x$ and $y$, as expected.

\subsubsection{Footprint for Long-Range Interactions}

Contrary to the head-on interaction where the small amplitude particles are mostly affected, now the large amplitude particles experience the strongest long-range beam-beam perturbations. This is rather intuitive since the large amplitude particles are the ones which can come closest to the opposing beam as they perform their oscillations. We must therefore expect a totally different tune footprint. Such a footprint for only long-range interactions is shown in Fig. 4.26.

\subsubsection{Studies of Long Range Interactions in the LHC}

To study the effect of long range beam-beam interactions we have performed a dedicated experiment [172]. The LHC was set up with single trains of 36 bunches per beam, spaced by $50 \mathrm{~ns}$. The bunch intensities were $\sim 1.2 \times 10^{11} \mathrm{p} / \mathrm{b}$ and the normalized emittances around $2.5 \mu \mathrm{m}$. The trains collided in IP1 and IP5, leading to a maximum of 16 long range encounters per interaction point for nominal bunches. First, the crossing angle (vertical plane) in IP1 was decreased in small steps and the 


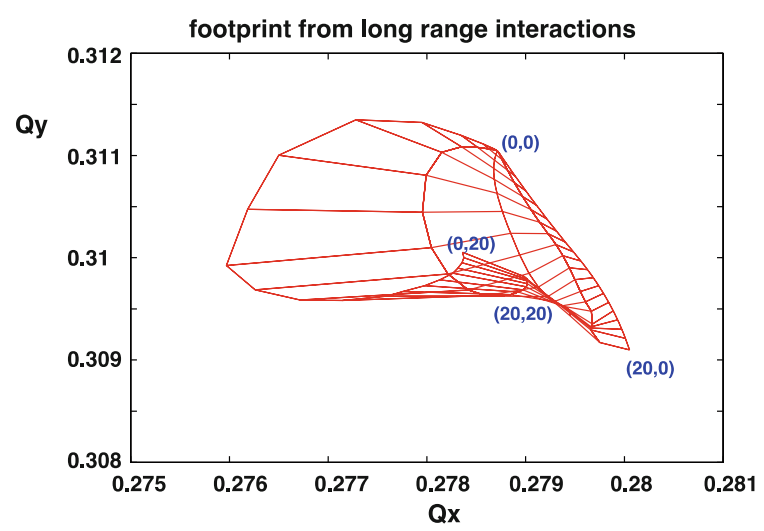

Fig. 4.26 Tune footprint for long-range interactions only. Vertical separation and amplitudes between 0 and $20 \sigma$

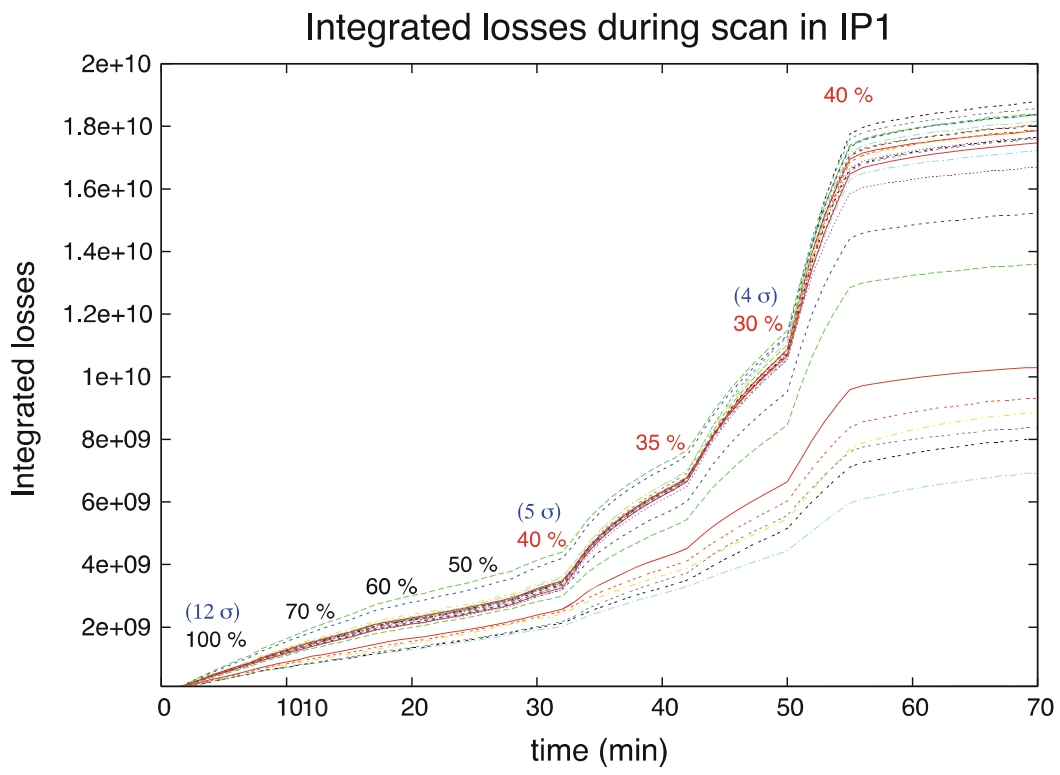

Fig. 4.27 Integrated losses of all bunches as a function of time during scan of beam separation in IP1. Numbers show percentage of full crossing angle

losses of each bunch recorded. The details of this procedure are described in [173] and the results are shown in Fig. 4.27 where the integrated losses for the 36 bunches in beam 1 are shown as a function of time and the relative change of the crossing angle is given in percentage of the nominal $(100 \% \equiv 240 \mu \mathrm{rad})$. The nominal value corresponds to a separation of approximately $12 \sigma$ at the parasitic encounters. From Fig. 4.27 we observe significantly increased losses for some bunches when the separation is reduced to about $40 \%$, i.e. around $5 \sigma$. Not all bunches are equally 


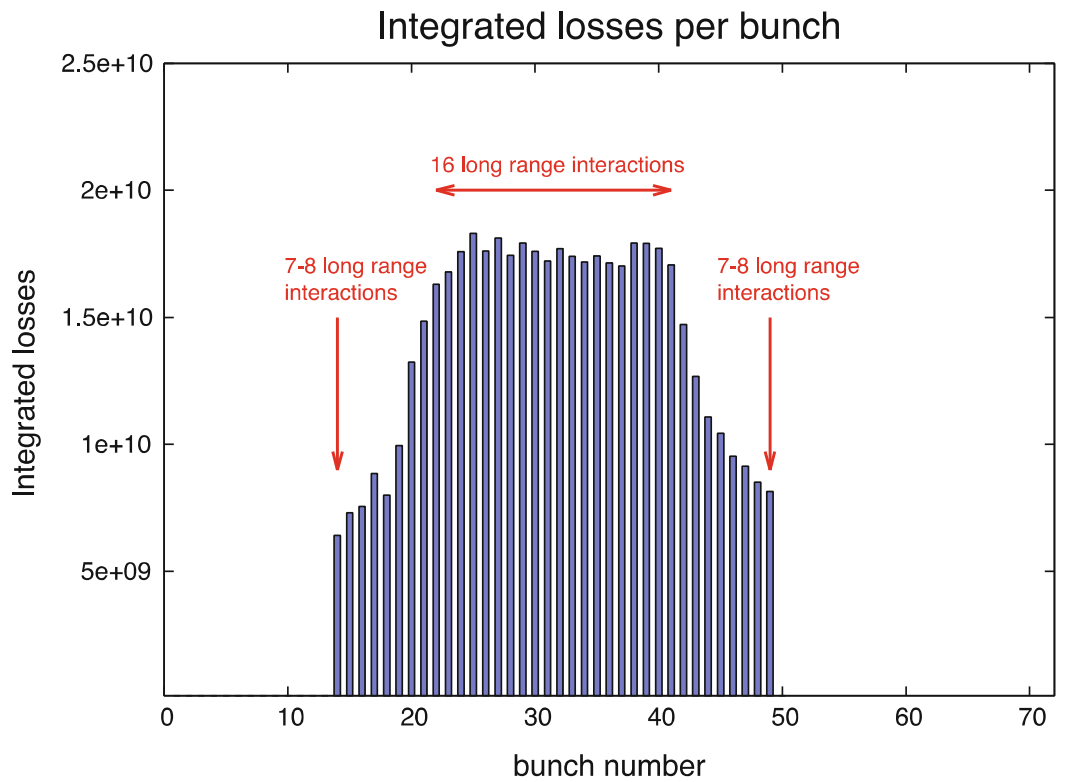

Fig. 4.28 Integrated losses of all bunches along a train of 36 bunches, after reducing the crossing angle in IP1

affected. At a smaller separation of $30 \%$ all bunches experience significant losses $(\sim 4 \sigma)$. Returning to a separation of $40 \%$ reduces the losses significantly, suggesting that mainly particles at large amplitudes have been lost during the scan due to a reduced dynamic aperture. Such a behaviour is expected [174]. The different behaviour is interpreted as a "PACMAN" effect and should depend on the number of long range encounters, which varies along the train. This is demonstrated in Fig. 4.28 where we show the integrated losses for the 36 bunches in the train at the end of the experiment. The maximum loss is clearly observed for the bunches in the centre of the train with the maximum number of long range interactions (16) and the losses decrease as the number of parasitic encounters decrease. The smallest loss is found for bunches with the minimum number of interactions, i.e. bunches at the beginning and end of the train $[166,167]$. This is a very clear demonstration of the expected different behaviour, depending on the number of interactions.

In the second part of the experiment we kept the separation at $40 \%$ in IP1 and started to reduce the crossing angle in the collision point IP5, opposite in azimuth to IP1. Due to this geometry, the same pairs of bunches meet at the interaction points, but the long range separation is in the orthogonal plane. This alternating crossing scheme was designed to compensate first order effects from long range interactions [166]. The Fig. 4.29 shows the evolution of the luminosity in IP1 as we performed the scan in IP5. The numbers indicate again the relative change of separation, this time the horizontal crossing angle in IP5. The luminosity seems to show that the lifetime is best when the separation and crossing angles are equal for 




Fig. 4.29 Luminosity in IP1 as a function of time during scan of beam separation in IP5

the two collision points. It is worse for smaller as well as for larger separation. This is the expected behaviour for a passive compensation due to alternating crossing planes, although further studies are required to conclude.

\subsubsection{Dynamic Aperture Reduction Due to Long-Range Interactions}

For too small separation, the tune spread induced by long-range interactions can become very large and resonances cannot be avoided any more. The motion can become irregular and as a result particles at large amplitudes may be lost.

To evaluate the dynamic aperture in the presence of beam-beam interactions, a simulation of the complete machine is necessary and the interplay between the beam-beam perturbation and possible machine imperfections is important [174].

For the present LHC parameters we consider the minimum crossing angle to be $285 \mu \mathrm{rad}$.

\subsubsection{Beam-Beam Induced Orbit Effects}

When two beams do not collide exactly head-on, the force has a constant contribution which can easily be seen when the kick $\Delta x^{\prime}$ from (4.65), for sufficiently large 
separation, is developed in a series

$$
\Delta x^{\prime}=\frac{\text { const }}{d}\left[1-\frac{x}{d}+O\left(\frac{x^{2}}{d^{2}}\right)\right]
$$

A constant contribution, i.e. more precisely an amplitude independent contribution, changes the orbit of the bunch as a whole. When the beam-beam effect is strong enough, i.e. for high intensity and/or small separation, the orbit effects are large enough to be observed.

When the orbit of a beam changes, the separation between the beams will change as well, which in turn will lead to a slightly different beam-beam effect and so on. The orbit effects must therefore be computed in a self-consistent way [175], in particular when the effects are sizeable. The closed orbit of an accelerator can usually be corrected, however an additional effect which is present in some form in many colliders, sets a limit to the correction possibilities. A particularly important example is the LHC and therefore it will be used to illustrate this feature.

We have to expect a slightly different orbit from bunch to bunch. The bunches in the middle of a train have all interactions and therefore the same orbit while the bunches at the beginning and end of a train show a structure which exhibits the decreasing number of long-range interactions. The orbit spread is approximately $10-15 \%$ of the beam size. Since the orbits of the two beams are not the same, it is impossible to make all bunches collide exactly head-on. A significant fraction will collide with an offset. Although the immediate effect on the luminosity is small [162], collisions at an offset can potentially affect the dynamics and are undesirable. The LHC design should try to minimize these offsets $[168,176]$. A further consequence of the LHC filling and collision scheme is that not all bunches experience all head-on collisions [176]. Some of the bunches will collide only in 2 instead of the 4 nominal interaction points, leading to further bunch-to-bunch differences. In Fig. 4.30 we show a prediction for the vertical offsets in IP1 [166, 167]. The offsets should vary along the bunch train. Although the orbit measurement in the LHC is not able to resolve these effects, the vertex centroid can be measured bunch by bunch in the experiment (Fig. 4.31).

\subsubsection{Coherent Beam-Beam Effects}

So far, we have mainly studied how the beam-beam interaction affects the single particle behaviour and treated the beam-beam interaction as a static lens. In the literature, this is often called a "weak-strong" model: a "weak" beam (a single particle) is perturbed by a "strong" beam (not affected by the weak beam). When the beam-beam perturbation is important, the model of an unperturbed, strong beam is not valid anymore since its parameters change under the influence of the other beam and vice versa. When this is the case, we talk about so-called "strong-strong" conditions. The first example of such a "strong-strong" situation was the orbit effect 


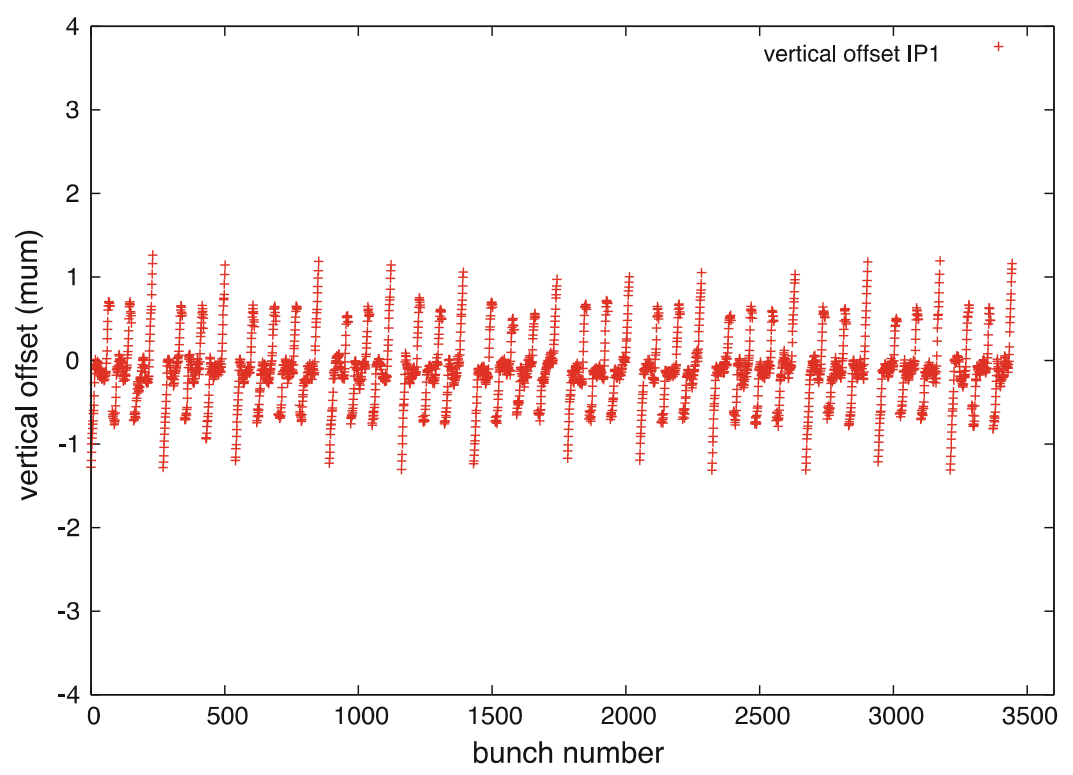

Fig. 4.30 Computed orbit offsets in IP1 along the bunch train [166, 167]

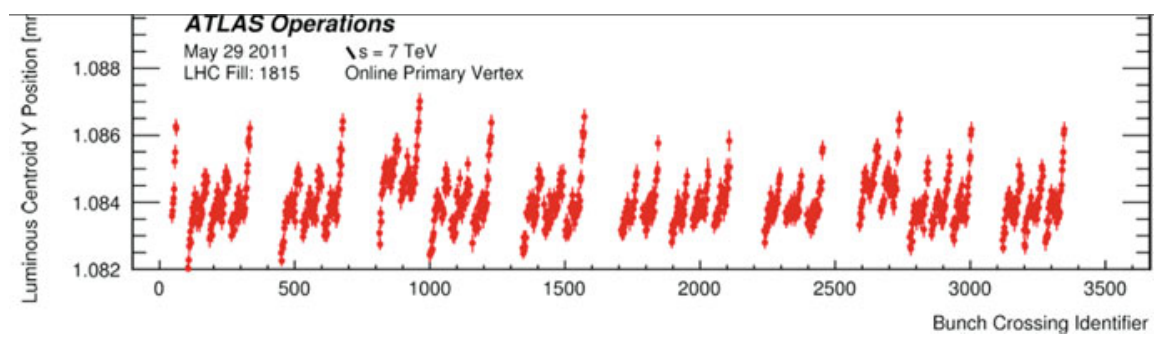

Fig. 4.31 Measured orbit offsets in IP1 along the bunch train [177, 178]

where the beams mutually changed their closed orbits. These closed orbits had to be found in a self-consistent way. This represents a static strong-strong effect.

In the next step, we investigate dynamic effects under the strong-strong condition [179].

When we consider the coherent motion of bunches, the collective behaviour of all particles in a bunch is studied. A coherent motion requires an organized behaviour of all particles in a bunch. A typical example are oscillations of the centre of mass of the bunches, so-called dipole oscillations. Such oscillations can be driven by external forces such as impedances and may be unstable. At the collision of two counter-rotating bunches not only the individual particles receive a kick from the opposing beam, but the bunch as an entity gets a coherent kick. This coherent kick of separated beams can excite coherent dipole oscillations. Its strength depends on the distance between the bunch centres at the collision point. It can be computed 


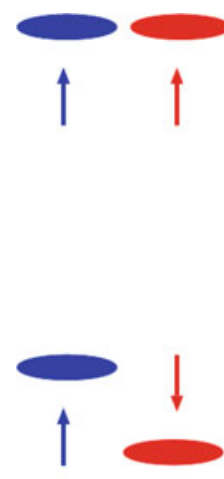

TURN n
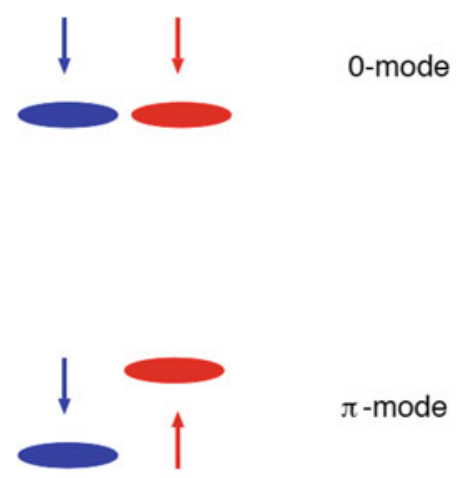

TURN $n+1$

Fig. 4.32 Basic dipole modes of two bunches. Relative position of the bunches at the interaction point at two consecutive turns

by adding the individual contributions of all particles. For small distances, it can be shown $[153,180]$ that it is just one half of the incoherent kick a single particle would receive at the same distance. For distances large enough the incoherent and coherent kicks become the same.

\subsubsection{Coherent Beam-Beam Modes}

To understand the dynamics of dipole oscillations we first study the simplest case with one bunch in each beam. When the bunches meet turn after turn at the collision point, their oscillation can either be exactly in phase ( 0 degree phase difference) or out of phase (180 degrees or $\pi$ phase difference). Any other oscillation can be constructed from these basic modes. The modes are sketched very schematically in Fig. 4.32. The relative positions of the bunches as observed at the interaction point are shown for two consecutive turns $n$ and $n+1$. The first mode is called the 0 mode (or sometimes called $\sigma$-mode) and the second the $\pi$-mode. In the first mode, the distance between the bunches does not change turn by turn and therefore there is no net force driving an oscillation. This mode must oscillate with the unperturbed frequency (tune) $Q_{0}$. For the second mode, the net force difference between two turns is a maximum and the tune becomes $Q_{0}+\Delta Q_{c o h}$. The sign of $\Delta Q_{c o h}$ depends whether the two beams have equal charge (defocusing case) or opposite charge (focusing case). The calculation of $\Delta Q_{c o h}$ is non-trivial: when the bunches are considered as rigid objects, the tune shift can be computed easily using the coherent kick but is underestimated [181]. The correct calculation must allow for changes of the density distribution during the collision and moreover, must allow a deviation from a Gaussian function. The computation requires to solve the Vlasov-equation of two coupled beams [182-185]. 
Self-consistent multi-particle simulations form a complementary study, but require large computing resources. Furthermore, the fields produced by the beams must be computed in a self-consistent form before every collision [186].

The 0 -mode is found at the unperturbed tune as expected. The $\pi$-mode is shifted by $1.2-1.3 \xi$. The precise value depends on the ratio of the horizontal and vertical beam sizes [183]. We have seen before the incoherent tune spread (footprint) the individual particles occupy and we know that it spans the interval $[0.0,1.0] \xi$, starting at the 0 -mode.

Here one can make an important observation: under the strong-strong condition the $\pi$-mode is a discrete mode outside the incoherent spectrum [184, 185]. This has dramatic consequences for the stability of the beams. A coherent mode that is outside an incoherent frequency spectrum cannot be stabilized by Landau damping. Under these conditions the coherent beam-beam effect could drive the dipole oscillation to large amplitudes and may result in the loss of the beam. Observations of the coherent beam-beam effects have been made at PETRA [181]. Beam-beam modes have been observed with high intensity coasting beams in the ISR [187], and recently in a bunched hadron collider at RHIC [188].

Coherent beam-beam modes can be driven by head-on collisions with a small offset or by long-range interactions. In the first case and for small oscillations, the problem can be linearized and the theoretical treatment is simplified. The forces from long-range interactions are very nonlinear but the numerical evaluation is feasible. Since the coherent shift must have the opposite sign for long-range interactions, the situation is very different. In particular the $\pi$-mode from long-range interactions alone would appear on the opposite side of the 0 -mode in the frequency spectrum [185, 186]. Both, the incoherent and the coherent spectra include both types of interactions.

\subsubsection{Compensation of Beam-Beam Effects}

For the case the beam-beam effects limit the performance of a collider, several schemes have been proposed to compensate all or part of the detrimental effects. The basic principle is to design correction devices which act as non-linear "lenses" to counteract the distortions from the non-linear beam-beam "lens". For both headon and long-range effects schemes have been proposed

- Head on effects:

- Electron lenses

- Linear lens to shift tunes

- Non-linear lens to decrease tune spread

- Long-range effects:

- At large distance: beam-beam force changes like $1 / \mathrm{r}$

- Same force as a wire! 


\subsubsection{Electron Lenses}

The basic principle of a compensation of proton-proton (or antiproton) collisions with an "electron lens" implies that the proton (antiproton) beam travels through a counter-rotating high current electron beam ("electron lens") $[189,190]$. The negative electron space charge can reduce the effect from the collision with the other proton beam.

An electron beam with a size much larger than the proton beam can be used to shift the tune of the proton beam ("linear lens"). When the current in the electron bunches can be varied fast enough, the tune shift can be different for the different proton bunches, thus correcting PACMAN tune shifts.

When the electron charge distribution is chosen to be the same as the counterrotating proton beam, the non-linear focusing of this proton beam can be compensated ("non-linear lens"). When it is correctly applied, the tune spread in the beam can be strongly reduced.

Such lenses have been constructed at the Tevatron at Fermilab [190] and experiments are in progress.

\subsubsection{Electrostatic Wire}

To compensate the tune spread from long-range interactions, one needs a non-linear lens that resembles a separated beam. At large enough separation, the long-range force changes approximately with $1 / r$ and this can be simulated by a wire parallel to the beam [191].

In order to compensate PACMAN effects, the wires have to be pulsed according to the bunch filling scheme. Tests are in progress at the SPS to study the feasibility of such a compensation for the LHC.

\subsubsection{Möbius Scheme}

The beam profiles of $\mathrm{e}^{+} \mathrm{e}^{-}$colliders are usually flat, i.e. the vertical beam size is much smaller than the horizontal beam size. Some studies indicate that the collision of round beams, even for $\mathrm{e}^{+} \mathrm{e}^{-}$colliders, show more promise for higher luminosity since larger beam-beam parameters can be achieved. Round beams can always be produced by strong coupling between horizontal and vertical planes. A more elegant way is the so-called Möbius lattice [192, 193]. In this lattice, the horizontal and vertical betatron oscillations are exchanged by an insertion. A horizontal oscillation in one turn becomes a vertical oscillation in the next turn and vice versa. Tests with such a scheme have been done at CESR at Cornell [193]. 


\subsection{Numerical Modelling}

\section{G. Rumolo}

Collective effects can be studied analytically, either through the perturbation formalism applied to the Vlasov equation or by means of few (typically two or three) particles models with the basic ingredients such as to reproduce the essential features of the phenomenon under study. Both ways are usually based on simplified approaches, in which some assumptions are necessary to make the models analytically solvable and lead to limited sets of equations relatively easy to interpret and handy to use.

The analytically solvable two or three particles models can be refined further to more realistic models, in which more than just few particles are assumed to represent the full particle beam. However, since the number of coupled differential equations to be solved grows proportionally with the number of particles used in the model, the resulting set of equations will rapidly become unmanageable as we increase the number of macroparticles (which are used to approximate the beam with a reduced number of particles), unless it is fed into a numerical simulation to be run on a computer. By using computers, the number of macroparticles necessary to model a beam can be pushed up to several millions, which is very useful to study the details of all possible internal oscillation modes of a bunch (or train of bunches), and also incoherent effects like emittance growth. Although ideally we would like to develop simulation programs that take into account the highest possible number of effects, in practice the existing codes narrow down their models to one or few effects, whose consequences in the beam dynamics are interesting to single out. For example, to study the effects of electron clouds, the beam will be made to interact with a given electron cloud at some locations around the accelerator ring, but in general other possible interactions with impedances, or the concurrent effects of space charge, beam-beam, Intra Beam Scattering, will be neglected. Although the study of two or more effects simultaneously is technically possible in most cases, at the present state of art of the simulations, it is generally preferred to limit the study of such interplays, because the combined models are difficult to control and tend to break down. Pushing further on this line, not only different effects can be decoupled in simulations, but also different regimes can be studied separately in the beam dynamics. For instance, for some problems only a partial description of the beam will be sufficient, so that transverse problems can be treated separately from longitudinal problems as well as single-bunch/multi-turn effects can be studied ignoring that these bunches are parts of long trains. In some cases, singlebunch/single- or multi-turn effects can also be modelled to generate driving terms to be used in reduced studies extending over longer time scales.

To perform a simulation, we will therefore have to define our beam as an ensemble of macroparticles, identified through arrays containing the phase space coordinates of each macro-particle (2-6-dimensional). This beam is first initialized and then transported across selected points of the accelerator ring using the 
appropriate transformation matrices. At each of these points, the interaction with the desired collective effect will be applied (e.g. the beam's own space charge field, an electron cloud, a wake field). It is clear, therefore, that a numerical simulation requires in the first place the knowledge of the driving term to be applied at each interaction point. That is why in the following we separate the general simulation into the solution of an electromagnetic problem, in which the collective interaction is modelled and the resulting excitation on the beam is calculated (at least, its nonself-consistent part), and the beam tracking part, in which the evolution of a beam is studied under the effect of this excitation. Note that most of numerical simulations including collective effects are based on time domain models, as these are best suited to describe the usually non-stationary beam evolution under the effect of collective interactions.

\subsubsection{The Electromagnetic Problem}

The first step to set up a numerical simulation including a collective effect consists of identifying the source of the self-induced perturbation acting on the beam and modelling it in a way that can be subsequently used. We usually distinguish three different types of collective interactions, which can take place with: (i) space charge (see Sect. 4.1); (ii) wake fields from an accelerator component or part of the resistive beam pipe (see Sect. 4.2); (iii) another "beam" of charged particles. This secondary beam can be either a counter-rotating beam in a collider (see Sect. 4.6), or a static cloud formed by the accumulation of particles, usually of opposite charge, around the primary beam (see Sect. 4.5).

If the source of the perturbation is space charge, then two different approaches are possible to compute its effect. Analytical formulae are available for the electromagnetic fields of coasting beams with ellipsoidal or Gaussian transverse sizes, as well as of ellipsoidal or Gaussian bunches (in all dimensions). The additional kicks given by these electromagnetic fields can be therefore calculated and applied to the beam macroparticles in a finite number of locations along the ring (even if the space charge interaction is in reality continuous). When doing that, self-consistency requires that the sizes of the bunch are updated at every kick point. Another possible approach consists of using the macroparticle distributions at each selected kick point to calculate self-consistently the electric field with a Poisson solver and use it to calculate the electromagnetic kicks on the macroparticles. It is worth noting that the same approach can be used for beam-beam problems, because the shape of the required electric field is the same, even if the coefficients need to be adapted (electric and magnetic forces tend to cancel at ultra-relativistic energies for space charge, while they add up for beam-beam).

If the source of the perturbation is a wake field from an accelerator component or resistive wall, the shape of the relative wake function has to be calculated beforehand. This is done analytically for some specific cases (e.g. resistive wall, step or tapered transitions), but in general dedicated electromagnetic codes can be 
used for this purpose. Some of them work in time domain, and can provide the wake potentials for given source bunches (usually chosen to be short enough as to simulate ideal pulse excitations and thus provide directly the wake functions). Other work in frequency domain and output impedances, which need then to be back-transformed into time domain to obtain the wake functions.

If the source of the perturbation is for example an electron cloud, then the electron distribution of the cloud is usually calculated beforehand by means of an electron cloud build-up code, and then its interaction with a coming bunch is calculated. Programs tracking simultaneously electrons and beam particles are presently under development or test, due to the massive memory and CPU requirements to solve this type of problems. On the other hand, generation and tracking of the ions can be included in multi-bunch beam tracking programs to calculate the effect of ions on bunched electron beams in a fully self-consistent manner. This is due to the fact that, while ions do not move significantly during the passage of an electron beam and allow modelling the bunches as charged disks, electrons can even perform several oscillations during the passage of a bunch, which requires a much more detailed modelling of the bunch.

\subsubsection{Beam Dynamics}

Beam dynamics tracking codes simulate the motion of beam particles inside an accelerator by transporting them across a number of discrete points by means of transformation matrices. In each of these points, additional kicks can be added, modelling either nonlinear components and errors of the external fields or the collective interactions. In the previous subsection, we have outlined the procedure to calculate the excitation to be applied to the beam to compute its evolution when it feels one or more collective interactions. To model the effects of space charge, wake fields and electron clouds, it is certainly necessary to describe the beam as an ensemble of macroparticles but beside that, its longitudinal structure needs also to be detailed. In particular, to model coupled bunch instabilities the relative positions of the macroparticles across the different bunches are necessary to determine the total effect of the wake acting on each of them. For single-bunch effects, a possible technique is to subdivide the bunch into several slices, so that the macroparticles of each slice can feel the integrated effect of the wakes left behind by the preceding slices (or the space charge from its own and the neighbouring slices, or the electron cloud as was deformed by the previous slices). A possible scheme of numerical simulation of a single bunch under the effect of a longitudinal wake field is illustrated in Fig. 4.33.

The bunch is first divided into $N$ slices and a kick must be applied to each macroparticle within a given slice at a certain kick point. The kick depends on the longitudinal wake function and the charge distribution of the preceding slices. In the longitudinal plane, particles within a slice feel also the effect of the same slice to which they belong, because the bunch suffers a net energy loss. After all 

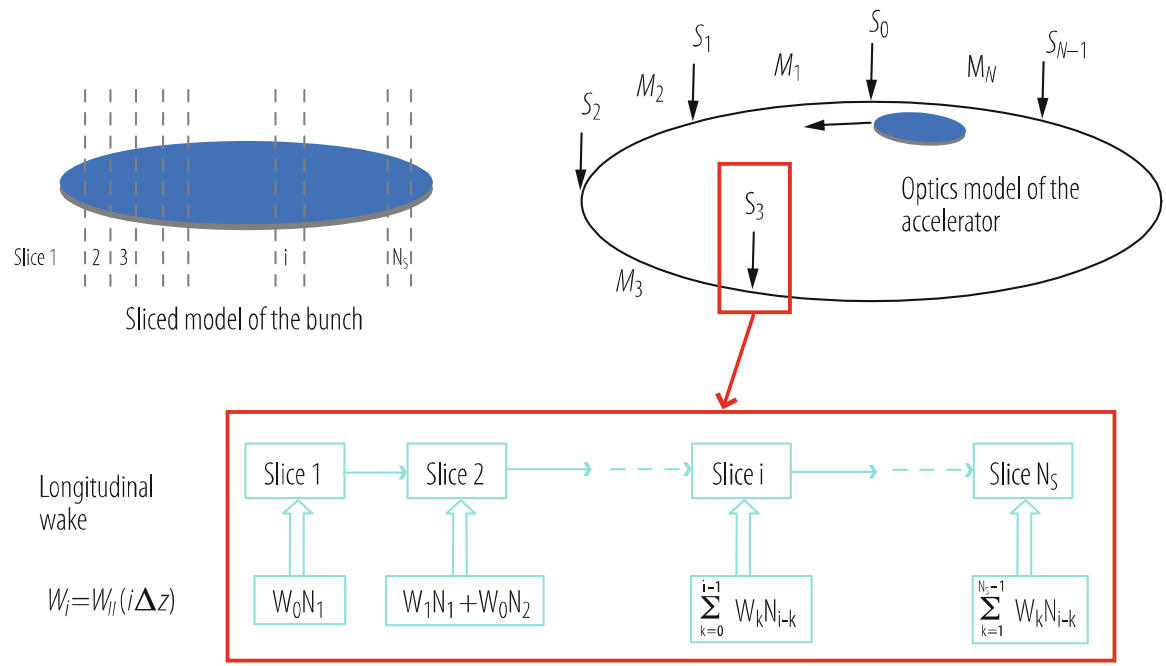

Fig. 4.33 Example of numerical simulation with collective effects: schematic view of the interaction of a single bunch with a longitudinal wake field at several locations in an accelerator ring

the particles in the bunch feel the effect of the wake at the kick point, they are subsequently transported to the next kick point in the accelerator optical model. Since synchrotron motion plays a key role in most of the effects under study, it is essential that particles are made to execute their synchrotron oscillations and move across slices from turn to turn. This means that collective effects dealing with singlebunch problems need to have at least one model of synchrotron motion built in, and that the bunch binning has to be regularly updated. Since the synchrotron motion is slow enough, and in reality the RF cavities do really kick the beam particles once or few times per turn, the longitudinal coordinates and the bunch slicing are usually not updated more frequently than once per turn. However, the update of the longitudinal coordinates from kick to kick point within one single turn, based on the only drift from momentum spread, could become significant especially in space charge simulations.

The simulation scheme with transverse wakes is basically the same as the one displayed in Fig. 4.33, except that particles inside one slice do not feel the effect of the same slice (as the transverse wakes are zero in the origin, for ultra-relativistic particles) and dipolar and quadrupolar contributions can be separated, making the wake kicks depending not only on the position of the source slice but also on the position of the witness macroparticles. The simulation scheme with the electron cloud is again similar to the one shown in Fig. 4.33, but the fundamental difference is that there is a mutual action between beam and electron cloud, so that, while macroparticles within a slice feel the effect of the electron cloud, the electron cloud itself is also deformed by the action of the passing slice. 
The modelling described in the previous subsections has been frequently applied to explain collective instabilities observed in running machines, as well as to predict instability thresholds (both in existing and future machines) and develop strategies to circumvent limitations from collective effects. For instance, a detailed impedance model of the SPS comprises the contributions from several accelerator components and is used for deriving single-bunch wake fields, which are the driving terms for HEADTAIL simulations. The kicks given to the beam particles by the different wake fields can be then either applied at the real locations in which the sources are situated, or weighted by the beta functions, summed up and applied in a single location using a one-kick approximation. These simulations can be used for predicting at which intensity transverse mode coupling occurs and the effects of chromaticity on this threshold value [194]. This is very important to extrapolate the beam stability limits in different conditions of operation, e.g. with a different optics or to the upgraded machine, which will be in principle enabled to receive higher intensity bunches. The mode shift can be plotted as a function of the bunch intensity, because the main modes are detectable from the Fourier analysis of the centroid motion. A typical plot of mode shift provided by simulations is displayed in Fig. 4.10.

\section{References}

1. E. Métral et al., Beam Instabilities in Hadron Synchrotrons, IEEE Transactions on Nuclear Science, Vol. 63, No. 2, 50 p, April 2016 (invitation for the 50th anniversary of the PAC conference).

2. E. Métral (Issue Editor), ICFA Beam Dynamics Newsletter No. 69 devoted to the Collective Effects in Particle Accelerators, 310 p, December 2016.

3. E. Métral and V.G. Vaccaro (chairs), ICFA Mini-Workshop on "Electromagnetic Wake Fields and Impedances in Particle Accelerators", Erice (Sicily, Italy), 2014: https://indico.cern.ch/ event $/ 287930 /$

4. M.R. Masullo, S. Petracca and G. Rumolo (chairs), ICFA Mini-Workshop on "Impedances and Beam Instabilities in Particle Accelerators", Benevento (Italy), 2017: https:// agenda.infn.it/event/12603/

5. E. Métral, G. Rumolo and T. Pieloni (chairs), ICFA Mini-Workshop on "Mitigation of Coherent Beam Instabilities in Particle Accelerators", Zermatt (Switzerland), 2019: https:// indico.cern.ch/event/775147/

6. B. Zotter: Betatron Frequency Shifts due to Image and Self Fields, CERN Accelerator School: General Accelerator Physics, CERN 85-19, Vol. I, p. 253, 1985.

7. K. Schindl: Space Charge, CERN-PS-99-012-DI, 1999.

8. A.W. Chao: Physics of Collective Beam Instabilities in High Energy Accelerators, New York: Wiley, $371 \mathrm{p}, 1993$.

9. K.Y. Ng: Physics of Intensity Dependent Beam Instabilities, World Scientific, 776 p, 2006.

10. S. Andriamonje, et al.: Neutron TOF facility (PS213): Technical Design Report, CERNINTC-2000-004, 2000.

11. E. Métral, Some effects near transition, Proceedings of the CAS-CERN Accelerator School on Intensity Limitations in Particle Beams on November 2015, CERN Yellow Reports: School Proceedings, CERN-2017-006-SP.

12. E. Métral and G. Rumolo, USPAS course on "Collective Effects in Beam Dynamics" in Albuquerque, New Mexico, USA, June 22-26, 2009: http://emetral.web.cern.ch/emetral/ 
13. W. Hardt, D. Möhl: Q-jump at Transition, CERN ISR-300/GS/69-16, 1969.

14. D. Möhl: Compensation of Space Charge Effects at Transition by a Asymmetric Q-jump $-A$ Theoretical Study, CERN ISR-300/GS/69-62, 1969.

15. W. Hardt: Proc. 9th Intern. Conf. High Energy Accelerators, Washington DC, 1974.

16. E. Métral and D. Möhl, Transition Crossing, Fifty years of the CERN Proton Synchrotron Volume I (editors: S. Gilardoni and D. Manglunki), CERN-2011-004, 16 June 2011.

17. J.S. Berg, F. Ruggiero: Landau Damping with Two-Dimensional Betatron Tune Spread, CERN SL-AP-96-71 (AP), December 1996.

18. E. Métral, F. Ruggiero: Stability Diagrams for Landau Damping with Two-Dimensional Betatron Tune Spread from both Octupoles and Nonlinear Space Charge Applied to the LHC at Injection, Proc. EPAC2004, Lucerne, Switzerland, 5-9 July 2004.

19. G. Franchetti, et al.: Space Charge and Octupole Driven Resonance Trapping Observed at the CERN Proton Synchrotron, Phys. Rev. ST Accel. Beams 6, 124201 (2003).

20. G. Franchetti: Simulations of the Space Charge and Beam Loss in the SIS18 Experiment, Proc. ICFA HB2010, Morschach, Switzerland, Sept. 27-Oct. 1, 2010.

21. B.W. Montague: Fourth-Order Coupling Resonance Excited by Space-Charge Forces in a Synchrotron, CERN 68-38, 1968.

22. E. Métral: Space Charge Experiment at the CERN Proton Synchrotron, Proc. ICFA HB2004, Bensheim, Germany, 18-22/10/2004.

23. L.J. Laslett: On Intensity Limitations Imposed by Space Charge Effects in Circular Particle Accelerators, in: Proc. Summer Study on Storage Rings, Accelerators and Instrumentation at Super High Energies, BNL Report 7534, p. 324, 1963.

24. K. Yokoya: Resistive Wall Impedance of Beam Pipes of General Cross Section, Part. Accel., Vol. 41, 3-4, p. 221, 1993.

25. V.G. Vaccaro, Longitudinal Instability of a Coasting Beam Above Transition, due to the Action of Lumped Discontinuities, CERN Rep. ISRRF/66-35, Tech. Rep., 1966.

26. A. Sessler and V. Vaccaro, CERN Report ISR-RF/67-2 (1967).

27. H. Tsutsui: On single Wire Technique for Transverse Coupling Impedance Measurement, CERN-SL-Note-2002-034 AP, 2002.

28. A. Burov, V. Danilov: Suppression of Transverse Bunch Instabilities by Asymmetries in the Chamber Geometry, Phys. Rev. Lett. 82, 2286-2289 (1999).

29. N. Mounet, E. Métral: Impedances of Two Dimensional Multilayer Cylindrical and Flat Chambers in the Non-Ultrarelativistic case, Proc. ICFA HB2010, Morschach, Switzerland, Sept. 27-Oct. 1, 2010.

30. CST Particle Studio. http://www.cst.com/Content/Products/PS/Overview.aspx

31. F. Caspers: Bench Measurements, in: Handbook of Accelerator Physics and Engineering, 2nd printing, edited by A.W. Chao and M. Tigner, p. 574.

32. Ansoft - HFSS. http://www.ansoft.com/products/hf/hfss/

33. E. Métral, et al.: Kicker Impedance Measurements for the Future Multiturn Extraction of the CERN Proton Synchrotron, Proc. EPAC2006, Edinburgh, UK, 26-30 June 2006.

34. H.A. Day: Measurements and Simulations of Impedance Reduction Techniques in Particle Accelerators, PHD Thesis, University of Manchester (England), 2013.

35. L.J. Laslett, V.K. Neil, A.M. Sessler: Transverse Resistive Instabilities of Intense Coasting Beams in Particle Accelerators, R.S.I 36(4), 436-448, 1965.

36. B. Zotter: New Results on the Impedance of Resistive Metal Walls of Finite Thickness, CERNAB-2005-043, 2005.

37. E. Métral: Transverse Resistive-Wall Impedance from Very Low to Very High Frequencies, CERN-AB-2005-084, 08 August 2005.

38. A.M. Al-Khateeb, et al.: Transverse Resistive Wall Impedances and Shielding Effectiveness for Beam Pipes of Arbitrary Wall Thickness, Phys. Rev. ST Accel. Beams 10, 064401 (2007).

39. F. Roncarolo, et al.: Comparison Between Laboratory Measurements, Simulations, and Analytical Predictions of the Transverse Wall Impedance at Low Frequencies, Phys. Rev. ST Accel. Beams 12, 084401 (2009). 
40. N. Mounet, E. Métral: Impedances of an Infinitely Long and Axisymmetric Multilayer Beam Pipe: Matrix Formalism and Multimode Analysis, Proc. 1st Intern. Particle Accelerator Conf., Kyoto, Japan, 23-28 May 2010.

41. N. Mounet, E. Métral: Generalized Form Factors for the Beam Coupling Impedances in a Flat Chamber, Proc. 1st Intern. Particle Accelerator Conf., Kyoto, Japan, 23-28 May 2010.

42. B. Salvant, et al.: Quadrupolar Transverse Impedance of Simple Models of Kickers, Proc. 1st Intern. Particle Accelerator Conf., Kyoto, Japan, 23-28 May 2010.

43. C. Zannini, et al.: Electromagnetic Simulations of Simple Models of Ferrite Loaded Kickers, Proc. 1st Intern. Particle Accelerator Conf., Kyoto, Japan, 23-28 May 2010.

44. E. Métral: Beam Screen Issues (with 20 T dipole magnets instead of 8.3 T), Proc. High-Energy LHC workshop, Malta, 14-16 October 2010.

45. A. Hofmann: Beam Instabilities, CERN Accelerator School, Rhodes, Greece, 20 Sept.-1 Oct., 1993, p. 307.

46. N. Biancacci, B. Salvant: Dependence of Impedance vs. Phase Advance in HEADTAIL, https://indico.cern.ch/event/164172/contributions/1420072/attachments/196173/275289/1912-2011_Impedance-Vs-PhaseAdvance.pdf

47. N. Biancacci, HEADTAIL + MAD-X, https://indico.cern.ch/event/178920/contributions/ 1446485/attachments/235706/329825/HDTL_lattice_def.pdf

48. E.D. Courant, A.M. Sessler: Transverse Coherent Resistive Instabilities of Azimuthally Bunched Beams in Particle Accelerators, R.S.I, 37(11), 1579-1588, 1966.

49. C. Pellegrini, Nuovo Cimento, 64 $, 477,1969$.

50. M. Sands: The Head-Tail Effect: an Instability Mechanism in Storage Rings, SLAC-TN-69-8, 1969.

51. M. Sands: Head-Tail Effect II: From a Resistive-Wall Wake, SLAC-TN-69-10, 1969.

52. F. Sacherer: Single beam collective phenomena-transverse (bunched beams), Proc. First Course Intern. School on Accelerators, Erice, 1976: Theoretical Aspects of the Behaviour of Beams in Accelerators and Storage Rings, CERN 77-13, p. 210, 1977.

53. J.L. Laclare: Bunched Beam Coherent Instabilities, CERN Accelerator School, Oxford, CERN 87-03, p. 264, 1987.

54. J.P. Gourber, H.G. Hereward, S. Myers: Overlap Knock-Out Effects in the CERN Intersecting Storage Rings (ISR), IEEE Trans. Nucl. Sci. NS-24(3), June 1977.

55. S. Myers: private communication (2011).

56. K.W. Robinson: Cambridge Electron Accel. Report CEAL-1010 (1964).

57. J.L. Laclare: Introduction to Coherent Instabilities - Coasting Beam Case, CERN Accelerator School: General Accelerator Physics, CERN 85-19, Vol. II, p. 377, 1985.

58. E. Keil, W. Schnell: CERN Report ISR-TH-RF/69-48, 1969.

59. D. Boussard: Observation of Microwave Longitudinal Instabilities in the CPS, CERN Report LabII/RF/Int./75-2, 1975.

60. F.J. Sacherer: Bunch Lengthening and Microwave Instability, IEEE Trans. Nucl. Sci. NS24(3), June 1977.

61. K. Ng: Potential-Well Distortion and Mode-Mixing Instability in Proton Machines, FERMILAB-FN-630, 1995.

62. E. Métral: Stability Criterion for the Longitudinal Mode-Coupling Instability in the Presence of Both Space-Charge and Resonator Impedances, CERN/PS 2001-063 (AE), 2001.

63. E. Métral, GALACTIC and GALACLIC: Two Vlasov Solvers for the Transverse and Longitudinal Planes, Proceedings of IPAC2019, Melbourne, Australia, May 19-24, 2019.

64. E. Métral and M. Migliorati, Longitudinal Mode-Coupling Instability: GALACLIC Vlasov Solver vs. Macroparticle Tracking Simulations, Proceedings of IPAC2019, Melbourne, Australia, May 19-24, 2019.

65. E. Shaposhnikova: Signatures of Microwave Instability, CERN-SL-99-008 HRF, 1999.

66. J.S. Berg, R.D. Ruth: Transverse Instabilities for Multiple Nonrigid Bunches in a Storage Ring, Phys. Rev. E 52(3), September 1995.

67. H. Hereward: Rutherford Lab. Reports RL-74-062, EPIC/MC/48 (1974), and RL-75-021, EPIC/MC/70 (1975). See also B. Chen, A. W. Chao: SSCL Report 606 (1992). 
68. D. Boussard, T. Linnecar: Proc. 2nd Euro. Part. Accel. Conf, Nice, 1990, p. 1560.

69. R.D. Kohaupt: Head Tail Turbulence and the Transverse PETRA Instability, DESY 80/22, 1980.

70. E. Métral: Stability Criteria for High-Intensity Single-Bunch Beams in Synchrotrons, Proc. 8th EPAC, Paris, France, 3-7 June 2002.

71. A. Burov and T. Zolkin, TMCI with Resonator Wakes, arXiv:1806.07521v2 [physics.acc-ph].

72. G. Rumolo et al., Simulation Study on the Energy Dependence of the TMCI Threshold in the CERN-SPS, Proceedings of EPAC 2006, Edinburgh, Scotland.

73. Y.H. Chin: User's guide for new MOSES version 2.0: Mode-coupling Single bunch instability in an Electron Storage ring, CERN/LEP-TH/88-05, 1988.

74. G. Rumolo, F. Zimmermann: Practical user guide for HEADTAIL, CERN-SL-Note-2002036-AP, 2002.

75. B. Salvant: Impedance Model of the CERN SPS and Aspects of LHC Single-Bunch Stability, PHD Thesis, Ecole Polytechnique Fédérale de Lausanne (Switzerland), 2010.

76. A. Burov, Nested Head-Tail Vlasov Solver, Phys. Rev. ST Accel. Beams 17, 021007 (2014).

77. N. Mounet, DELPHI: An Analytic Vlasov Solver for Impedance-Driven Modes, CERN internal HSC meeting, May 07, 2014.

78. E. Métral, G. Rumolo: Simulation Study on the Beneficial Effect of Linear Coupling for the Transverse Mode Coupling Instability in the CERN Super Proton Synchrotron, Proc. EPAC 2006, Edinburgh, UK, 26-30 June 2006.

79. S. Myers: LEP Note 436, 1983.

80. R. Ruth: CERN LEP-TH/83-22, 1983.

81. R. Ruth: Proc. 12th Intern. Conf. High Energy Accelerators, Fermilab, Batavia, August 1116, 1983, p. 389.

82. S. Myers: J. Vancraeynest: CERN LEP-RF/84-13, 1984.

83. S. Myers: CERN LEP-RF/85-22, 1985.

84. S. Myers: Proc. IEEE PAC, Washington D.C., March 16, 1987, p. 503-507.

85. M. Karliner, K. Popov: Theory of a Feedback to Cure Transverse Mode Coupling Instability, Nucl. Instrum. Meth. A 537 (2005), p. 481-500.

86. E. Métral et al., Destabilising Effect of the LHC Transverse Damper, in Proc. IPAC'18, Vancouver, BC, Canada, Apr-May 2018, paper THPAF048, pp. 3076-3079. https://doi.org/ 10.18429/JACoW-IPAC2018-THPAF048

87. E. Métral et al., Impedance Models, Operational Experience and Expected Limitations, International Review of the HL-LHC Collimation System, CERN, 11-12/02/2019.

88. E. Métral, A Two-Mode Model to Study the Effect of Space Charge on TMCI in the "LongBunch" regime, Proceedings of IPAC2019, Melbourne, Australia, May 19-24, 2019.

89. E. Métral et al., Space Charge and Transverse Instabilities at the CERN SPS and LHC, Proceedings of ICAP' 18, Key West, Florida, USA, October 20-24, 2018.

90. A. Burov, "Convective Instabilities of Bunched Beams with Space Charge", arXiv: $1807.04887 \mathrm{v} 4$ [physics.acc-ph].

91. E. Métral, Summary of my 3 IPAC19 papers, CERN LIU-SPS BD WG meeting, 17/01/2019.

92. H.G. Hereward: Landau Damping by Non-Linearity, CERN/MPS/DL 69-11, 1969.

93. E. Métral, A. Verdier: Stability Diagrams for Landau Damping with a Beam Collimated at an Arbitrary Number of Sigmas, CERN-AB-2004-019-ABP.

94. E. Métral, Landau Damping for TMCI: Without vs. With Transverse Damper (TD), CERN HSC internal meeting, 25/03/2019, https://indico.cern.ch/event/807899/contributions/ 3362767/attachments/1816203/2971974/LDforTMCI_EM_25-03-2019_2.pdf

95. E. Métral: Theory of Coupled Landau Damping, Part. Accelerators 62(3-4), p. 259, 1999.

96. E. Métral et al., Simulation Study of the Horizontal Head-Tail Instability Observed at Injection of the CERN Proton Synchrotron, Proceedings of PAC07, Albuquerque, New Mexico, USA, June 25-29, 2007.

97. E. Métral, et al.: Destabilising Effect of Linear Coupling in the HERA Proton Ring, Proc. 8th EPAC, Paris, France, 3-7 June 2002. 
98. L.R. Carver et al., Transverse Beam Instabilities in the Presence of Linear Coupling in the Large Hadron Collider, PRAB 21, 044401 (2018).

99. E. Métral: Simple Theory of Emittance Sharing and Exchange due to Linear Betatron Coupling, CERN/PS 2001-066 (AE), December 2001.

100. A. Franchi, E. Métral, R. Tomás: Emittance Sharing and Exchange Driven by Linear Betatron Coupling in Circular Accelerators, Phys. Rev. ST Accel. Beams 10, 064003 (2007).

101. D. Möhl, H. Schönauer: Landau Damping by Non-Linear Space-Charge Forces and Octupoles, Proc. IX Intern. Conf. High Energy Acc., Stanford, 1974 (AEC, Washington, D.C., 1974), [CONF 740522] p. 380.

102. D. Möhl: On Landau Damping of Dipole Modes by Non-Linear Space Charge and Octupoles, CERN/PS 95-08 (DI), 1995.

103. M. Blaskiewicz: Phys. Rev. ST Accel. Beams 1, 044201 (1998).

104. E. Métral, F. Ruggiero: Stability Diagrams for Landau Damping with Two-Dimensional Betatron Tune Spread from both Octupoles and Nonlinear Space Charge, CERN-AB-2004025 (ABP), 2004.

105. A. Burov: Phys. Rev. ST Accel. Beams 12, 044202 (2009).

106. V. Balbekov: Phys. Rev. ST Accel. Beams 12, 124402 (2009).

107. V. Kornilov: Head-Tail Bunch Dynamics with Space Charge, Proc. ICFA HB2010, Morschach, Switzerland, Sept. 27-Oct. 1, 2010.

108. X. Buffat et al., Transverse Mode Coupling Instability of Colliding Beams, Phys. Rev. ST Accel. Beams 17, 041002 (2014).

109. X. Buffat, Transverse Beams Stability Studies at the Large Hadron Collider, Ph.D. dissertation, EPFL, 2015.

110. S.V. Furuseth et al., MD3288: Instability latency with controlled noise, CERN internal note, under publication.

111. S. Nagaitsev, et al.: Nonlinear Optics as a Path to High-Intensity Circular Machines, Proc. ICFA HB2010, Morschach, Switzerland, Sept. 27-Oct. 1, 2010.

112. V. Shiltsev et al., Landau Damping of Beam Instabilities by Electron Lenses, Phys. Rev. Lett. 119, 134802 - Published 27 September 2017.

113. M. Schenk et al., Analysis of Transverse Beam Stabilization with Radio Frequency Quadrupoles, PRAB 20, 104402 (2017).

114. M. Schenk et al., Experimental Stabilization of Transverse Collective Instabilities in the LHC with Second Order Chromaticity, PRAB 21, 084401 (2018).

115. M. Schenk et al., Vlasov Description of the Effects of Nonlinear Chromaticity on Transverse Coherent Beam Instabilities, PRAB 21, 084402 (2018).

116. E. Métral: Stability of the Longitudinal Bunched-Beam Coherent Dipole Mode, Proc. APAC'04, Gyeongju, Korea, 22-26 March 2004.

117. K. Ohmi: Phys. Rev. Lett. 75, 1526 (1995).

118. Tables and Graphs of electron-Interaction Cross Sections from $10 \mathrm{eV}$ to $100 \mathrm{GeV}$ Derived from the LLNL Evaluated Electron Data Library (EDLL), Z=1-100, UCRL-50400, 31 (1991).

119. T. Raubenheimer, P. Chen: Ions in the Linacs of Future Linear Colliders, Proc. LINAC'92, Ottawa, Canada (1992).

120. P. Thieberger, et al: Phys. Rev. A 61, 042901 (2000).

121. O. Grobner: Bunch Induced Multipacting, 10th Intern. Conf. High Energy Accelerators, Protvino, July (1977).

122. R. Cimino, et al.: Phys. Rev. Lett. 93, 014801 (2004).

123. M. Furman, G. Lambertson: The Electron-Cloud Instability in PEP-II, Proc. EPAC'96, Sitges (1996).

124. F. Zimmermann: LHC Project Report 95 (1997).

125. L. Wang, et al.: PRST-AB 5, 124402 (2002).

126. H. Fukuma, et al.: Observation of Vertical Beam Blow-up in KEKB Low Energy Ring, Proc. EPAC'00, Vienna (2000). 
127. G. Arduini, et al.: Transverse Behaviour of the LHC Proton Beam in the SPS: An Update, PAC2001, Chicago (2001).

128. K. Cornelis: The Electron Cloud Instability in the SPS, ECLOUD'02, Geneva (2002).

129. C. Yin Valgren, et al.: Amorphous Carbon Coatings for Mitigation of Electron Cloud in the CERN SPS, Proc. 1st Intern. Particle Accelerator Conf., Kyoto, Japan, 23-28 May 2010.

130. G. Rumolo et al., Electron Cloud observation in the LHC, Proc. IPAC'11, San Sebastian, Spain (2011).

131. G. Iadarola, Electron cloud studies for CERN particle accelerators and simulation code development, $\mathrm{PhD}$ thesis, CERN-THESIS-2014-047 (2014).

132. L. Mether et al., Electron cloud in 2016: cloudy or clear?, Proc. 7th Evian Workshop, Evian, France, 2016, CERN-ACC-2017-094 (2017).

133. G. Arduini et al., How to Maximize the HL-LHC performance, Proc. of the Review of the LHC and Injector Upgrade Plans Workshop (RLIUP), 29-31 October 2013 Archamps, France, CERN-2014-006 (2013)

134. M. Palmer, et al.: Electron Cloud at Low Emittance in CesrTA, Proc. 1st Intern. Particle Accelerator Conf., Kyoto, Japan, 23-28 May 2010.

135. V. Baglin, et al.: LHC Project Report 472, (2002).

136. M. Furman, G. Lambertson: The Electron-Cloud Instability in the Arcs of the PEP-II Positron Ring, Proc. MBI97 Tsukuba (1997).

137. P. Channell, A. Jason: LA AOT-3 TN 93-11, private communication (1994).

138. P.R. Zenkevich, D.G. Koskarev: Coupling Resonances of the Transverse Oscillations of Two Circular Beams, ITEF-841, (1970), translated as UCRL-Trans. 1451 (1971).

139. E. Keil, B. Zotter: CERN-ISR-TH/71-58 (1971).

140. K. Oide, et al., in: Proc. International Workshop on Performance Improvement of ElectronPositron Collider Particle Factories, KEKB Report No. 99-24 (2000).

141. K. Ohmi, F. Zimmermann: Phys. Rev. Lett. 85, 3821 (2000)

142. G. Arduini, et al., in: Proc. Workshop LEP-SPS Performance Chamonix X (2000), pp. 119 122.

143. G. Arduini, et al., in: Proc. PAC 2003, 12-16 May, Portland, USA (2003).

144. K. Li et al., Beams during the cycle: Quality and behaviour, LHC Performance Workshop 2017, Chamonix, France (2017).

145. A. Romano et al., Electron cloud instabilities triggered by low bunch intensity at the Large Hadron Collider, presently submitted to PRAB.

146. D. Neuffer, et al: Observations of a fast transverse instability in the PSR, Nucl. Instrum. Meth. A 321, pp. 1-12 (1992).

147. A. Grunder, G.R. Lambertson: Report UCRL 20691 (1971).

148. G. Rumolo, in: Proc. CARE-HHH-APD Workshop, CERN (2004).

149. G. Rumolo, et al.: Phys. Rev. Lett. 100, 144801 (2008).

150. Proc. ECLOUD02, CERN, Geneva, 2002, edited by G. Rumolo and F. Zimmermann as CERN Yellow Report No. CERN-2002-001 (2002).

151. R. Cimino et al., Phys. Rev. Lett., 109, 064801 (2012).

152. F. Ruggiero, X.L. Zhu: Collective Instabilities in the LHC: Electron Cloud and Satellite Bunches, Proc. Workshop Instabilities of High Intensity Hadron Beams in Rings, Upton, NY, USA, 28 Jun-1 Jul 1999, pp. 40-48.

153. W. Herr: Beam-Beam Interactions, Proceedings of CERN Accelerator School, Zeuthen 2003, CERN-2006-002 (2006).

154. Proceedings of the ICFA-Mini-Workshop on Beam-Beam effects in Hadron Colliders, CERN, Geneva, Switzerland, 18-22 April 2013, edited by W. Herr and G. Papotti, CERN-2014-004, https://doi.org/10.5170/CERN-2014-004

155. A. Chao: The Beam-Beam Instability, SLAC-PUB-3179 (1983).

156. L. Evans, J. Gareyte: Beam-Beam Effects, CERN Accelerator School, Oxford 1985, CERN 87-03 (1987).

157. A. Zholents: Beam-Beam Effects in Electron-Positron Storage Rings, Joint US-CERN School on Particle Accelerators, in Springer, Lecture Notes in Physics, Vol. 400 (1992). 
158. E. Keil: Beam-Beam Dynamics, CERN Accelerator School, Rhodes 1993, CERN 95-06 (1995).

159. S. Kheifets, PETRA-Kurzmitteilung 119 (DESY 1976).

160. M. Basetti and G.A. Erskine, Closed Expression for the Electrical Field of a 2-Dimensional Gaussian Charge, CERN-ISR-TH/80-06 (1980).

161. D. Brandt, et al.: Is LEP Beam-Beam Limited at its Highest Energy? In Proceedings of the Particle Accelerator Conference 1999, New York, (1999) p. 3005.

162. W. Herr: Concept of Luminosity, Proceedings of CERN Accelerator School, Zeuthen 2003, CERN-2006-002 (2006).

163. J.T. Seeman: Observations of the Beam-Beam Interaction, Lecture Notes in Physics, Vol. 247, Springer, (1986).

164. S. Myers: IEEE Trans. Nucl. Sci. NS-28, 2503 (1981).

165. S. Myers: Review of beam-Beam Simulations, Lecture Notes in Physics, Vol. 247, Springer, (1986).

166. W. Herr, Features and Implications of Different LHC Crossing Schemes, CERN LHC Project Report 628 (2003).

167. W. Herr, Dynamic Behaviour of Nominal and PACMAN Bunches for Different LHC Crossing Schemes, CERN LHC Project Report 856 (2005).

168. W. Herr, Effects of PACMAN Bunches in the LHC, CERN LHC Project Report 39 (1996).

169. W. Herr et al., Head-On Beam-Beam Tune Shifts with High Brightness Beams in the LHC, CERN-ATS-Note-2011-029 (2011).

170. G. Papotti et al., Observations of Bunch to Bunch Differences due to Beam-Beam Effects, Proceedings of IPAC2011, San Sebastián, Spain.

171. B. Goddard, et al.: Bunch Trains for LEP, Particle Accelerators 57, 237 (1998).

172. W. Herr et al., Observations of beam-beam effects at high intensities in the LHC, In Proceedings of the Particle Accelerator Conference 2011, San Sebastion (2011).

173. W. Herr et al., Head-on beam-beam interactions with high intensities and long range beambeam studies in the LHC, CERN-ATS-Note-2011-058 (2011).

174. W. Herr, D. Kaltchev et al., Large Scale Beam-beam Simulations for the CERN LHC using distributed computing, Proc. EPAC 2006, Edinburgh (2006).

175. H. Grote, W. Herr: Self-Consistent Orbits with Beam-Beam Effects in the LHC, In Proceedings of the 2001 Workshop Beam-Beam Effects, FNAL, 25-27/6/2001.

176. W. Herr, Consequences of Periodicity and Symmetry for the Beam-Beam Effects in the LHC, CERN LHC Project Report 49 (1996).

177. W. Kozanecki and J. Cogan, private communication (2011).

178. R. Bartoldus, Online determination of the LHC Luminous Region with the ATLAS High Level trigger, TIPP 2011, Int. Conf. on Tech. and Instr. in Particle Physics, Chicago (2011).

179. Y. Alexahin, et al., Coherent Beam-Beam Effects in the LHC, Proc. HEACC 2001, Tsukuba, Japan, CERN LHC Project Report 466 (2001).

180. K. Hirata: Nucl. Instrum. Meth. A 269, 7 (1988).

181. A. Piwinski: Observation of Beam-Beam Effects in PETRA, IEEE Trans. Nucl. Sci. NS-26, 4268 (1979).

182. R.E. Meller, R.H. Siemann: IEEE Trans. Nucl. Sci. NS-28, 2431 (1981).

183. K. Yokoya, et al., Tune Shift of Coherent Beam-Beam Oscillations, Part. Acc. 27, 181 (1990).

184. Y. Alexahin, On the Landau Damping and Decoherence of Transverse Dipole Oscillations in Colliding Beams, Part. Acc. 59, 43 (1996).

185. Y. Alexahin, A Study of the Coherent Beam-Beam Effect in the Framework of the Vlasov Perturbation Theory, Nucl. Instrum. Meth. A 380, 253 (2002).

186. W. Herr et al., A Hybrid Fast Multipole Method Applied to Beam-Beam Collisions in the Strong-Strong Regime, Phys. Rev. ST Accel. Beams 4, 054402 (2001).

187. J.P. Koutchouk, ISR Performance Report - A Numerical Estimate of the Coherent Beam-Beam Effect in the ISR, CERN ISR-OP/JPK-bm (1982).

188. W. Fischer, et al., Observation of Coherent Beam-Beam Modes in RHIC, BNL C-AD/AP/75 (2002). 
189. V.D. Shiltsev, et al., Compensation of Beam-Beam Effects in the Tevatron Collider with Electron Beams, In Proceedings of the Particle Accelerator Conference 1999, New York, p. 3728.

190. V.D. Shiltsev, et al., Considerations on Compensation of Beam-Beam Effects in the Tevatron with Electron Beams, Phys. Rev. ST Accel. Beams 2, 071001 (1999).

191. J.P. Koutchouk, et al., Correction of the Long-Range Beam-Beam Effect in LHC using ElectroMagnetic Lenses, In Proceedings of the Particle Accelerator Conference 1999, New York, p. 1681.

192. R. Talman, A Proposed Möbius Accelerator, Phys. Rev. Lett. 74, 1590 (1995).

193. S. Henderson et al., Investigation of the Möbius Accelerator at CESR, CBN 99-5 (1999).

194. LHC Injectors Upgrade Technical Design Report - Volume I: Protons, edited by J. Coupard et al., CERN-ACC-2014-0337

Open Access This chapter is licensed under the terms of the Creative Commons Attribution 4.0 International License (http://creativecommons.org/licenses/by/4.0/), which permits use, sharing, adaptation, distribution and reproduction in any medium or format, as long as you give appropriate credit to the original author(s) and the source, provide a link to the Creative Commons licence and indicate if changes were made.

The images or other third party material in this chapter are included in the chapter's Creative Commons licence, unless indicated otherwise in a credit line to the material. If material is not included in the chapter's Creative Commons licence and your intended use is not permitted by statutory regulation or exceeds the permitted use, you will need to obtain permission directly from the copyright holder. 